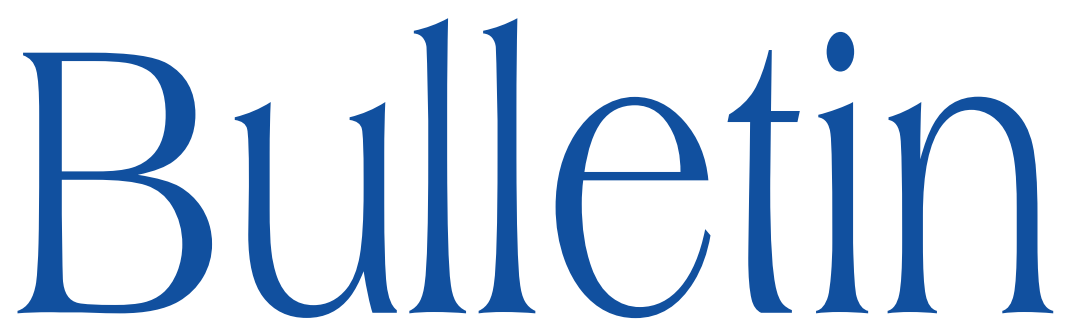

de la SOCIÉTÉ MATHÉMATIQUE DE FRANCE

\title{
CLASSIFICATION ANALYTIQUE DE STRUCTURES DE POISSON
}

\author{
Philipp Lohrmann
}

Tome 137

Fascicule 3

2009

SOCIÉTÉ MATHÉMATIQUE DE FRANCE

Publié avec le concours du Centre national de la recherche scientilque 
Bull. Soc. math. France

137 (3), 2009, p. 321-386

\title{
CLASSIFICATION ANALYTIQUE DE STRUCTURES DE POISSON
}

\author{
PAR PHILIPP LOHRMANN
}

\begin{abstract}
RÉsumé. - Notre étude porte sur une catégorie de structures de Poisson singulières holomorphes au voisinage de $0 \in \mathbb{C}^{n}$ et admettant une forme normale formelle polynomiale i.e. un nombre fini d'invariants formels. Les séries normalisantes sont divergentes en général. On montre l'existence de transformations normalisantes holomorphes sur des domaines sectoriels de la forme $a<\arg x^{R}<b$, où $x^{R}$ est un monôme associé au problème. Il suit une classification analytique.
\end{abstract}

Abstract (Analytic classification of Poisson structures). - Our study deals with some singular Poisson structures, holomorphic near $0 \in \mathbb{C}^{n}$ and admitting a polynomial normal form, i.e. a finite number of formal invariants. Their normalizing series generally diverge. We show the existence of normalizing transformations, holomorphic on some sectorial domains $a<\arg x^{R}<b$, where $x^{R}$ denotes a monomial associated to the problem. Follows an analytic classification.

\section{Introduction}

Étude locale des structures de Poisson. - Les structures de Poisson sont des généralisations naturelles du crochet de Poisson sur une variété symplectique :

Texte reçu le 8 janvier 2008, révisé le 4 novembre 2008 et le 6 février 2009, accepté le 14 mai 2009

Philipp Lohrmann, Universität Zürich, Institut für Mathematik, Winterthurerstr. 190, 8057

Zürich, Suisse • E-mail : philipp.lohrmann@math.uzh.ch

Classification mathématique par sujets (2000). — 58D27, 34M40, 40A05, 53D17, 32S99.

Mots clefs. - Phénomène de Stokes, singularités, sommabilité, formes normales, structures de Poissson. 
soient $M$ une variété analytique (pas nécessairement de dimension paire) et $C_{p}^{\omega}$ l'anneau des germes en $p \in M$ de fonctions holomorphes sur $M$. Une structure de Poisson sur $M$ est la donnée, en tout point $p \in M$, d'un crochet $\{.,\}:. C_{p}^{\omega} \times C_{p}^{\omega} \rightarrow C_{p}^{\omega}$ bilinéaire, antisymétrique tel que pour tout $f, g, h \in C_{p}^{\omega}$ les applications $\{f,$.$\} et \{., f\}$ soient des dérivations de $C_{p}^{\omega}$, et tel que l'identité de Jacobi $\{f,\{g, h\}\}+\{g,\{h, f\}\}+\{h,\{f, g\}\}=0$ soit satisfaite. En coordonnées locales une structure de Poisson est définie moyennant un champ de bivecteurs $\Pi=\sum_{i<j} \Pi_{i j}(x) \partial_{i} \wedge \partial_{j}$ où on pose $\partial_{i}:=\partial / \partial x_{i}$. Le crochet $\{f, g\}$ associé est la contraction du 2-tenseur contravariant $\Pi$ avec les 1-tenseur covariant $d f$ et $d g$ i.e.

$$
\{f, g\}=\sum_{i<j} \Pi_{i j}(x)\left(\partial_{i} f \partial_{j} g-\partial_{i} g \partial_{j} f\right) .
$$

Du fait de l'identité de Jacobi on doit avoir $\sum_{l=1}^{n}\left(\Pi_{i l} \partial_{l}\left(\Pi_{j k}\right)+\Pi_{j l} \partial_{l}\left(\Pi_{k i}\right)+\right.$ $\left.\Pi_{k l} \partial_{l}\left(\Pi_{i j}\right)\right)=0$ pour tout $1 \leq i, j, k \leq n$ ( $n$ désigne la dimension de la variété sous-jacente $M$ ). Soit $f \in C^{\omega}$. On appelle champ hamiltonien de $f$ relatif $\grave{a} \Pi$ le champ de vecteurs $X_{P} i$ tel que $\mathcal{L}_{X_{\Pi}}(g)=\{f, g\}$ pour tout $g \in C^{\omega}$, soit

$$
X_{\Pi}=\sum_{i<j} \Pi_{i j}\left[\partial_{i}(f) \partial_{j}-\partial_{j}(f) \partial_{i}\right]
$$

en coordonnées locales.

Remarque. - La différence d'une structure de Poisson et d'une structure symplectique consiste dans le fait que le rang d'une structure de Poisson $\Pi$ $\left(=\right.$ le rang de la matrice $\left.\left(\Pi_{i j}\right)\right)$ n'est pas nécessairement localement constant et peut différer de la dimension de la variété sous-jacente $M$. Dans ce cas ils existent des fonctions de Casimir, i.e. des fonctions non-constantes $f$ telles que $\{f,\}=$.0 .

L'étude locale des structures de Poisson se ramène à l'étude des structures de Poisson nulles à l'origine. En effet, A. Weinstein [22] a démontré un théorème à la Darboux pour les structures de Poisson : pour tout $p \in M$ il existe deux entiers $r$ et $s$ avec $n=2 r+s$, ainsi que des coordonnées holomorphes locales $\left(p_{1}, \ldots, p_{r}, q_{1}, \ldots, q_{r}, x_{1}, \ldots, x_{s}\right)$ telles que

$$
\Pi=\sum_{1 \leq i<j \leq r} \partial_{p_{i}} \wedge \partial_{q_{i}}+\sum_{1 \leq i<j \leq s} \pi_{i j}(x) \partial_{x_{i}} \wedge \partial_{x_{j}},
$$

où $\pi_{i j}$ désignent des fonctions holomorphes sur $\left(\mathbb{C}^{s}, 0\right)$ s'annulant en 0.

Les structures de Poisson qui s'annulent en $0 \in \mathbb{C}^{n}$ et qui apparaissent de manière structurellement stable se divisent en deux catégories : la première formée des structures de Poisson possédant une partie linéaire non-nulle qui sont de la forme $\Pi=\sum_{i, j} l_{i j}(x) \partial_{x_{i}} \wedge \partial_{x_{j}}+$ t.o.s., où $l_{i j}$ désigne des formes linéaires 
sur $\mathbb{C}^{n}$, et t.o.s. des termes d'ordre supérieur à l'origine. Ces structures de Poisson ont fait l'objet de nombreux études en catégorie formelle, holomorphe et $C^{\infty}$. Citons le célèbre résultat de J. Conn [4] en catégorie holomorphe et $C^{\infty}$ d'après lequel on a une conjugaison vers la partie linéaire si l'algèbre de Lie associé à la partie linéaire sur le cotangent est semi simple. Parmi les nombreux autre résultats mentionnons celui de Stolovitch [18] qui concerne la conjugaison holomorphe vers une forme normale dans certains cas non formellement linéarisables.

La deuxième classe de structures de Poisson structurellement stables est celle des structures de Poisson à 1-jet nul i.e. à partie linéaire nulle, mais à partie quadratique non nulle - de la forme $\Pi=\sum_{i, j} q_{i j}(x) \partial_{x_{i}} \wedge \partial_{x_{j}}+$ t.o.s., où on désigne par $q_{i j}$ des formes quadratiques sur $\mathbb{C}^{n}$. Après avoir étés étudiés la première fois par Arnold [1] ils ont fait l'objet de nombreuses études dans les années 90 - on renvoie le lecteur au livre de Dufour-Zung [6] et à la bibliographie qui s'y trouve.

En particulier, Dufour et Wade ont donné [5] une notion de forme normale formelle pour les structures de Poisson à 1-jet nul très similaire à celle de Poincaré-Dulac pour les champs de vecteurs - cf. section 2.

Classification analytique et formelle. - Dans [9], l'auteur de cet article à montré un théorème à la Bruno pour les structures de Poisson : soit $\Pi$ une structure de Poisson à 1-jet nul. Il existe un biholomorphisme $\Phi:\left(\mathbb{C}^{n}, 0\right) \rightarrow\left(\mathbb{C}^{n}, 0\right)$ tel que $\Phi^{*}(\Pi)$ soit une forme normale (au sens de Dufour-Wade) si deux conditions sont satisfaites :

- une condition de petits diviseurs diophantiens (D), associés à la partie quadratique de $\Pi$,

- une condition algébrique $(C)$ sur la forme normale formelle de $\Pi$ : l'ensemble des fonctions de Casimir relatifs à la forme normale formelle doit coïncider avec celui par rapport à la partie quadratique.

Une forme normale peut être considérée comme un représentant d'une classe de conjugaison. Si (C) et (D) sont satisfaites les classes de conjugaisons analytiques coïncident donc avec les classes de conjugaison formelles. Dans cet article on montre que si condition (C) n'est pas satisfaite, les séries normalisantes divergent en général. On donne (cf. section 6) un exemple d'une structure de Poisson très simple $\Pi$ qui ne satisfait pas condition (C) et qui admet une forme normale polynomiale. De plus toute transformation normalisant $\Pi$ diverge et la classification analytique ne se réduit pas à la classification formelle. L'objet de cet article est une classification analytique pour des structures de Poisson à un jet nul satisfaisant un certain nombre de conditions techniques. Les hypothèses faites ne sont pas les plus simples possibles, le but étant de montrer le phénomène de Stokes. 
Notre démarche pour la classification analytique est la suivante : dans le but d'avoir un nombre fini d'invariants formels, on cherche d'abord (cf. section 4) les conditions les plus simples possibles assurant une forme normale polynomiale : on considère des structures de Poisson de la forme $\Pi=\Pi^{2}+x^{R} \Pi^{R}+$ t.o.s. de partie quadratique $\Pi^{2}$ 1-résonnante - i.e. l'anneau des fonctions de Casimir formelles par rapport à la partie quadratique $\Pi^{2}$ est engendré par le monôme $x^{R}$, appelé générateur de résonances. On suppose $\Pi^{R}=S \wedge C$ où $S, C$ désignent des champs de vecteurs linéaires diagonaux tels que $\mathcal{L}_{S}\left(x^{R}\right)=0$ et $\mathcal{L}_{C}\left(x^{R}\right) \neq 0$. Les divergences des séries conjuguant $\Pi$ à la forme normale formelle $\Pi^{2}+x^{R} \Pi^{R}$ sont alors portées par $x^{R}$.

Pour assurer que le caractère de structure de Poisson soit préservé par multiplication par une fonction, on suppose que la forme normale $\Pi^{2}+x^{R} \Pi^{R}$ soit produit extérieur de deux champs de vecteurs. En effet, on ne pourra faire une classification qu'à multiplication par une unité analytique près.

Analyse. - On montre qu'ils existent des transformations normalisantes (avec un développement asymptotique divergent en $0 \in \mathbb{C}^{n}$ ) qui sont holomorphes sur certains domaines sectoriels $D S_{s}, s=0,1$, de la forme $a<\arg x^{R}<b$. Ces transformations nous permettent d'associer une isotropie sectorielle à chaque structure de Poisson appartenant à une classe de conjugaison formelle donnée. Le groupe des isotropies sectorielles, qui sert d'espace classifiant, consiste en les transformations holomorphes sur $D S_{0} \cap D S_{1}$ préservant la forme normale. Ils sont infiniment plats à l'origine.

Synthèse. - Une démarche, inventée par Martinet, Ramis et Malgrange [11] [12] permet de synthétiser une classe de conjugaison analytique à partir d'une isotropie sectorielle d'une forme normale donnée. Cette démarche est basée sur le théorème d'intégrabilité des structures presque-complexes de NewlanderNirenberg.

REMARQUE. - Les transformations normalisantes $\Phi_{s}, s=0,1$ qu'on donne sont les mêmes que ceux utilisées par Stolovitch dans [16] pour conjuguer certains champs de vecteurs vers une forme normale. Stolovitch a montré que ces transformations sont des limites de suites de transformations de la forme $\left(1+X_{i}\right)$, où chaque $X_{i}$ est la 1-somme (au sens de Ramis - cf. [14]) d'une série formelle divergente, sur un secteur d'ouverture strictement supérieur à $2 \pi$. Mais l'intersection ces secteurs n'est en général plus d'ouverture strictement supérieur à $2 \pi$. Ceci signifie que les transformations $\Phi_{s}$ ne sont en général plus des 1-sommes au sens de Ramis. En absence de certains petits diviseurs, Stolovitch et Braaksma ont montrés dans [2] que les transformations $\Phi_{s}, s=0,1$, sont des 1-sommes d'une série divergente. 
Ce travail s'inscrit dans la suite des travaux de Martinet-Ramis [11], [12], d'Ecalle-Voronin [7], [21] et de Stolovitch [16]. Il s'est fait dans le cadre d'un projet de thèse sous la direction de L. Stolovitch et l'auteur le remercie pour ses conseils et suggestions.

Guide de lecture, résumé. - Le résultat clef de cet article est le théorème 3.1 de normalisation sectorielle, énoncé en section 3. Il affirme que si la condition (C) n'est pas satisfaite on a sous des conditions (techniques) supplémentaires l'existence d'une conjugaison holomorphe vers une forme normale (polynomiale) sur certains domaines sectoriels $D S_{s}, s=0,1$, de la forme $a<\arg x^{R}<b$. La preuve de ce théorème occupe les sections 4 à 9 . On termine en montrant en section 10 comment on obtient à partir du théorème 3.1 des modules de conjugaison, i.e. une classification analytique.

Section 2 : notions préliminaires. - Dans cette section on rappelle quelques propriétés premières des structures de Poisson et on donne la notion de forme normale formelle de Dufour-Wade. De plus, on fixe des notations et on rappelle des notions de développement asymptotique sectoriels. Le lecteur est invité à consulter cette section uniquement au fur et à mesure des besoins.

Section 3 : théorème de normalisation sectorielle. - Quand la condition algébrique (C) sur la forme normale formelle n'est pas satisfaite, ils se pose, dans la perspective d'une classification analytique, la question suivante : sous quelles conditions a t'on un nombre fini d'invariants formels, i.e. l'existence d'une forme normale polynomiale. D'autre part il se pose la question de l'applicabilité de la théorie de la resommation des séries divergentes.

La théorie de la resommation des séries divergentes est actuellement limitée aux séries (divergentes) à une seule variable. Suivant une idée de L. Stolovitch [16], on se limite alors au cas 1-résonnant : on suppose l'anneau des fonctions de Casimir formels relatifs à la partie quadratique engendré par un seul monôme $x^{R}$, appelé le «générateur de résonances ». On assure ainsi que les divergences soient «portées » par un seul monôme $x^{R}$, ce qui permet (sous des conditions supplémentaires) de considérer les séries normalisantes comme des séries divergentes à une seule variable $z=x^{R}$, mais dépendant de paramètres.

Le cas le plus simple d'une structure de Poisson $\Pi$ à 1-jet nul, 1-résonnante et ne satisfaisant pas la condition $(\mathrm{C})$ est

$$
\Pi=\Pi^{2}+x^{R} \Pi^{R}+\text { t.o.s. }
$$

où $\Pi^{2}$ est la partie quadratique (1-résonnante), $x^{R}$ le générateur de résonances, et $\Pi^{R}=C \wedge S, C, S$ champs de vecteurs linéaires diagonaux tels que $\mathcal{L}_{C} x^{R} \neq 0$ et $\mathcal{L}_{S} x^{R}=0$. 
Le théorème 3.1 affirme qu'une structure de Poisson de la forme (1.2), satisfaisant certaines conditions techniques supplémentaires, est holomorphiquement conjuguée à la forme normale polynomiale $\Pi^{2}+x^{R} \Pi^{R}$ sur des domaines sectoriels $D S_{s}, s=0,1$, de la forme $a<\arg x^{R}<b$.

Section 4 : forme normale formelle polynomiale. - Le résultat principal de la section 4 est la proposition 4.1, d'après laquelle une structure de Poisson du type (1.2) est conjuguée à la forme normale polynomiale $\Pi^{2}+x^{R} \Pi^{R}$ par une transformation formelle.

La démonstration consiste essentiellement à montrer comment on passe d'une forme normale avec une infinité de termes résonnants (dont l'existence a été établi par Dufour et Wade dans [5]) à la forme normale polynomiale $\Pi^{2}+$ $x^{R} \Pi^{R}$. L'étape principale est la suivante : on suppose $\left[x^{R} \Pi^{R},\left(x^{R}\right)^{m} \Pi^{(m R)}\right]=0$, où $\Pi^{(m R)}$ désigne un champ de bivecteurs homogène de degré deux, et [., ] le crochet de Schouten (extension naturelle de la dérivée de Lie). Il s'agit alors de montrer l'existence d'un champ de vecteurs de la forme $\left(x^{R}\right)^{m} Z$, avec $Z$ champ de vecteurs linéaire, tel qu'on a $\left[x^{R} \Pi^{R},\left(x^{R}\right)^{m} Z\right]=\left(x^{R}\right)^{m} \Pi^{(m R)}$.

Section 5. - Certaines propriétés obtenues au cours de la démonstration de la proposition 4.1 nous permettent d'expliciter une structure de Poisson à 1-jet nul, de type (1.2), telle que toute série formelle normalisante est divergente.

Section 6 : prénormalisation à la Dulac. - Comme les séries normalisant une structure de Poisson $\Pi$ de type (1.2) sont généralement divergentes, il n'existe pas de transformation normalisable holomorphe définie sur un voisinage entier de l'origine. Néanmoins, on obtient dans la section 6 une première réduction $\mathrm{du}$ problème de classification : on y montre la proposition 6.1, qui affirme l'existence une transformation $\Phi$, holomorphe sur un voisinage de zéro, telle que la restriction de $\Phi^{*}(\Pi)$ à la variété $x^{R}=0$ est sous forme normale (plus précisément réduit à la partie quadratique). On dit alors que $\Pi$ est sous forme «prénormalisé ».

Ce résultat est la transposition aux structures de Poisson d'un résultat analogue de Stolovitch concernant les champs de vecteurs [15]. La preuve est basée sur des estimations fines du nombre de petits diviseurs. Dans le cas des structures de Poisson, la difficulté supplémentaire vient du fait que les équations cohomologiques admettent une solution uniquement dans le cas où une condition de compatibilité est satisfaite.

Dans le paragraphe consacré à la section 8 de ce guide de lecture on explique comment la proposition 7.2 est utilisé de façon essentielle lors de la preuve du théorème 3.1 de normalisation sectorielle. 
Section 7 : champ de vecteurs associé. - La question est la suivante : peut-on associer un champ de vecteurs formel $N$ à une structure de Poisson formelle $\Pi$ de type (1.2), tel que la conjugaison de $N$ vers une forme normale formelle polynomiale par une transformation formelle implique la conjugaison de $\Pi$ à la forme normale $\Pi_{\mathrm{n}}:=\Pi^{2}+x^{R} \Pi^{R}$ ?

L'existence de tels champs de vecteurs est suggéré par l'existence d'une réduction d'une structure de Poisson $\Pi$, obtenue par la mise sous forme normale (au sens de Poincaré-Dulac) d'un certain champ de vecteurs, appelé le rotationel, intrinsèquement associé à $\Pi$. Néanmoins on n'élimine pas ainsi tous les termes non-résonnants de $\Pi$ - pour plus de détails cf. [6], p.150-155.

En section 7 on montre que la question de l'existence de $N$ admet une réponse positive, à condition de se restreindre aux structures de Poisson $\Pi$ appartenant au $\mathbb{C}[x]$-module $D$ engendré par les champs de bivecteurs quadratiques diagonaux, c'est-à-dire les structures de Poisson $\Pi$ s'écrivant comme somme $\sum_{I} x^{I} \Pi^{I}$ avec $I$ ne comportant pas de composantes prenant la valeur -1 , et $\Pi^{I}$ champ de bivecteurs quadratique diagonal (somme de produits de champs de vecteurs linéaires diagonaux). Cette restriction est nécessaire pour assurer l'analyticité de $N$ en $0 \in \mathbb{C}^{n}$.

Sous une forme simplifiée, le résultat principal de la section 7, i.e. la proposition 7.2, s'énonce comme suit.

Proposition 1.1 (NF). - Soit $\Pi:=\Pi^{2}+x^{R} \Pi^{R}+\cdots \in D$ tel que dans 1.2. Alors pour $\Lambda \in \mathbb{C}^{* n}$ n'appartenant pas à une certaine réunion dénombrable d'hyperplans de $\mathbb{C}^{n}$, toute transformation formelle conjuguant le champ de vecteurs $\frac{1}{x^{\Lambda}} X_{\Pi}\left(x^{\Lambda}\right)$ à la forme normale polynomiale $\frac{1}{x^{\Lambda}} X_{\left[\Pi^{2}+x^{R} \Pi^{R}\right]}\left(x^{\Lambda}\right)$ conjugue également $\Pi$ à $\Pi_{\mathrm{n}}:=\Pi^{2}+x^{R} \Pi^{R}$.

REMARque. - Contrairement au cas du rotationnel, l'association $\Pi \rightarrow$ $\frac{1}{x^{\Lambda}} X_{\Pi}\left(x^{\Lambda}\right)$ n'est pas intrinsèque. Sous certaines hypothèses, la proposition 6.1 implique que la structure de Poisson prénormalisée appartient à $D$.

Section 8 : normalisation sectorielle. - En section 8 on montre le théorème 3.1. On prend comme point de départ une structure de Poisson $\Pi$ prénormalisée au sens de la proposition 6.1. Une division par une unité analytique adéquate $v_{\Lambda}$ permet alors que les champs de vecteurs associés $v_{\Lambda} \frac{1}{x^{\Lambda}} X_{\Pi}\left(x^{\Lambda}\right)$ soient « bien préparés » dans le sens de Stolovitch dans [16] (cf. définition 8.1). Ceci signifie que $v_{\Lambda} \frac{1}{x^{\Lambda}} X_{\Pi}\left(x^{\Lambda}\right)$ satisfait les hypothèses du théorème 3.3.1. dans [16], qui assure l'existence d'une transformation holomorphe $\Phi_{s}, s=0,1$, défini sur $D S_{s}$, conjuguant le champs de vecteurs $v_{\Lambda} \frac{1}{x^{\Lambda}} X_{\Pi}\left(x^{\Lambda}\right)$ vers la forme normale $\frac{1}{x^{\Lambda}} X_{\left[\Pi^{2}+x^{R} \Pi^{R}\right]}\left(x^{\Lambda}\right)$. 
Par la proposition (NF), $\Phi_{s}$ conjugue la structure de Poisson $v_{\Lambda} \Pi$ vers $\Pi_{\mathrm{n}}:=$ $\Pi^{2}+x^{R} \Pi^{R}$ sur $D S_{s}$, mais uniquement modulo un reste infiniment plat $\mathcal{R}_{j}^{\Lambda}$, i.e. sur $D S_{s}$ on a

$$
\Phi_{s}^{*}\left(v_{\Lambda} \Pi\right)=\Pi^{2}+x^{R} \Pi^{R}+\mathcal{R}_{s}^{\Lambda} .
$$

Remarque. - Si $P$ définit une structure de Poisson, i.e. si on a $[P, P]=$ 0 , il ne suit pas en général la relation $[u P, u P]=0$, avec $u$ fonction. Nous montrons que pour que le caractère de structure de Poisson soit préservé, il suffit de supposer la partie quadratique $\Pi^{2}$ produit extérieur de deux champs de vecteurs.

La difficulté principale est alors de montrer que $\mathcal{R}_{j}^{\Lambda}=0$ i.e. que $\Phi_{s}^{\Lambda}$ conjugue $\Pi$ à la forme normale $\Pi_{\mathrm{n}}=\Pi^{2}+x^{R} \Pi^{R} \operatorname{sur} D S_{s}, s=0,1$.

Le théorème 3.3.1. dans [16] affirme en plus que $\Phi_{j}^{\Lambda}$ admet $\widehat{\Phi}^{\Lambda}$ comme « développement asymptotique au sens Gérard-Sibuya » (défini en section 2). Par conséquent le reste infiniment plat $\mathcal{R}_{s}^{\Lambda}$ s'écrit

$$
\mathcal{R}_{s}^{\Lambda}=\frac{1}{2} \sum_{\substack{i \neq j \\ I \in \mathbb{D}}} x^{I} f_{i j s}^{I, \Lambda}\left(x^{R}\right) Y_{i} \wedge Y_{j}, \quad Y_{i}=x_{i} \partial / \partial x_{i},
$$

où $\mathbb{D}$ désigne l'ensemble des multi-indices tels que $x^{R}$ ne divise pas $x^{I}$ et les $f_{i j s}^{I, \Lambda}$ sont des fonctions infiniment plates définies sur un secteur de $\mathbb{C}$.

Revenons un instant au cas formel. Lors de la preuve de la proposition (NF) on suppose

$$
\Pi=\Pi^{2}+x^{R} \Pi^{R}+x^{I_{0}} \Pi^{I_{0}}+\cdots,
$$

où $x^{I_{0}} \Pi^{I_{0}}$ est un terme de plus petit degré qui est non résonnant. Par un calcul on déduit de la relation de compatibilité $\left[\Pi^{2}, x^{I_{0}} \Pi^{I_{0}}\right]=0$ que le champ de vecteurs $\frac{1}{x^{\Lambda}} X_{\left[x^{I_{0}} \Pi^{I_{0}}\right]}\left(x^{\Lambda}\right)$ est non nul.

L'idée pour montrer que $\mathcal{R}_{s}^{\Lambda}$ est nul est de faire le même calcul dans le cas où $\left(\Phi_{s}^{\Lambda}\right)^{*}\left(v_{\Lambda} \Pi\right)=\Pi^{2}+x^{R} \Pi^{R}+\mathcal{R}_{s}^{\Lambda}$, avec $\mathcal{R}_{s}^{\Lambda}$ tel qu'en (1.3), i.e.

$$
\left(\Phi_{s}^{\Lambda}\right)^{*}\left(v_{\Lambda} \Pi\right)=\Pi^{2}+x^{R} \Pi^{R}+x^{I_{0}} \Pi_{s}^{I_{0}, \Lambda}+\cdots,
$$

où ici $I_{0} \in \mathbb{D}$ et $\Pi_{s}^{I_{0}, \Lambda}=\sum_{i \neq j} f_{i j s}^{I_{0}, \Lambda}\left(x^{R}\right) Y_{i} \wedge Y_{j}$. Le calcul est identique à celui $\mathrm{du}$ cas formel puisque pour tout $1 \leq i \leq n$ on a la relation $\mathcal{L}_{A_{i}}\left(x^{R}\right)=0$, øù on a posé $A_{i}:=\sum_{j=1}^{n} a_{i j} Y_{j}, \Pi^{2}=\sum_{i \neq j} a_{i j} Y_{i} \wedge Y_{j}, a_{i j}=-a_{j i}$. Ceci permet de traiter les fonctions $f_{i j s}^{I_{0}, \Lambda}\left(x^{R}\right)$ comme des constantes. On montre ainsi que si $\mathcal{R}_{s}^{\Lambda}$ n'était pas nul on aboutirait à une contradiction avec le fait que le champ de vecteurs associé $\frac{1}{x^{\Lambda}} X_{\left[\Phi_{s}^{*}\left(v_{\Lambda} \Pi\right)\right]}\left(x^{\Lambda}\right)$ est sous forme normale. 
Section 9 : preuve de la proposition 8.2. - La difficulté principale dans le raisonnement précédent est la preuve de la proposition 8.2 qui affirme que l'identité de Jacobi $\left[\Phi_{s}^{*}\left(v_{\Lambda} \Pi\right), \Phi_{s}^{*}\left(v_{\Lambda} \Pi\right)\right]=0$ implique la relation de compatibilité $\left[\Pi^{2}, x^{I_{0}} \Pi_{s}^{I_{0}, \Lambda}\right]=0$. La démonstration de ce résultat, pour $\Lambda$ appartenant à un certain sous-ensemble de $\mathbb{C}$, fait l'objet de la section 9 .

La difficulté provient du fait qu'on raisonne modulo $x^{R}$ : il existe des paires de multi-indices $I^{\prime}, I^{\prime \prime} \in \mathbb{D}$ tels que $x^{I^{\prime}+I^{\prime \prime}}$ soit divisible par $x^{R}$, i.e. $I^{\prime}+I^{\prime \prime} \notin \mathbb{D}$ - il existe alors un entier $e \geq 1$ tel que $x^{I^{\prime}+I^{\prime \prime}} f\left(x^{R}\right)=x^{I^{\prime}+I^{\prime \prime}-e R} \tilde{f}\left(x^{R}\right)$, où on note $f, \tilde{f}$ des fonctions infiniment plates, et $I^{\prime}+I "-e R \in \mathbb{D}$. Le raisonnement ne peut donc plus s'effectuer sur le multi-indice $I_{0} \in \mathbb{D}$.

Dans une première étape on déduit des identités de Jacobi

$$
\left\{x^{\Lambda},\left\{x_{i}, x_{j}\right\}\right\}+\left\{x_{i},\left\{x_{j}, x^{\Lambda}\right\}\right\}+\left\{x_{j}\left\{x^{\Lambda}, x_{i}\right\}\right\}=0, \quad 1 \leq i, j \leq n,
$$

que pour tout $I$ fixé, les $\left(n(n-1)\right.$ fonctions infiniment plates $f_{i j s}^{I, \Lambda}$ vérifient un système différentiel linéaire à singularité irrégulière (cf. le lemme 9.1) (qui ne dépend uniquement de $\Lambda, \Pi^{2}$ et de $x^{R} \Pi^{R}$ ). Ensuite on en déduit que les fonctions $f_{i j s}^{I_{0}, \Lambda}$ sont des combinaisons $\mathbb{C}$-linéaires de termes de la forme

$$
f^{l, I, \Lambda}(u)=\log ^{l}(u)(u)^{G(I, \Lambda)} \exp \left(\frac{H(I, \Lambda)}{u}\right),
$$

où $G$ et $H$ sont certaines fonctions, dépendant de $I$ et de $\Lambda$. La preuve de la proposition 8.2 s'effectue alors en raisonnant sur les fonctions $H(I, \Lambda$ ) (et non pas, comme dans le cas classique sur le multi-indice $I$ ).

Section 10 : classification analytique. - Soient $\Pi^{2}$ et $x^{R} \Pi^{R}$ tels que dans (1.2) fixés. On suppose les conditions techniques supplémentaires du théorème 3.1 satisfaites. Le but est d'établir le théorème 10.2, c'est-à-dire donner une classification analytique, modulo multiplication par une unité analytique, de toutes les structures de Poisson de type (1.2), c'est-à-dire de la forme $\Pi=\Pi^{2}+x^{R} \Pi^{R}+$ t.o.s. (toutes conjuguées à la forme normale $\Pi^{2}+x^{R} \Pi^{R}$ par une transformation formelle).

Avec le théorème 3.1 on associe à chaque structure de Poisson analytique $\Pi$ l'isotropie sectorielle $\Phi_{i} \circ \Phi_{j}^{-1}$. L'espace des isotropies sectorielles est en bijection avec certains espaces de suites - cf. la proposition 10.2.

Pour montrer que les isotropies sectorielles sont des modules de conjugaison analytiques, il s'agit alors de résoudre le problème inverse, c'est-à-dire la synthétisation d'une structure de Poisson à partir de la donnée d'une isotropie sectorielle. Le résultat clef est ici le théorème 10.1, dont la démonstration, donnée dans Martinet-Ramis [11], est basée sur le théorème d'intégrabilité de Newlander-Nirenberg des structures presque complexes [13]. 


\section{Notions préliminaires}

Crochet de Schouten-Nijenhuis. - Désignons par $\chi_{1}$ les germes de champs de vecteurs en $0 \in \mathbb{C}^{n}$, et par $\chi_{p}:=\bigwedge^{p} \chi_{1}$, les germes de champs de $p$-vecteurs. On définit le crochet de Schouten-Nijenhuis [., .] $: \chi_{p} \times \chi_{q} \rightarrow \chi_{p+q-1}, \mathbb{C}$-bilinéaire, extension de la dérivée de Lie tel que

$$
\begin{aligned}
& {[P, Q]=-(-1)^{(p-1)(q-1)}[Q, P]} \\
& {[P, Q \wedge R]=[P, Q] \wedge R+(-1)^{(p-1) q} Q \wedge[P, R]} \\
& {\left[x^{J} \Pi, x^{K} \Pi^{\prime}\right]=2\left(X_{\Pi^{\prime}}\left(x^{J}\right) \wedge \Pi+X_{\Pi}\left(x^{K}\right) \wedge \Pi^{\prime}\right) .}
\end{aligned}
$$

Un champ de 2-vecteurs $\Pi$ définit une structure de Poisson, si et seulement si $[\Pi, \Pi]=0$ (identité de Jacobi). Désignons par $X_{\Pi}(f)$ le champ hamiltonien d'une fonction $f$ relatif à une structure de Poisson $\Pi$, pour lequel on a la relation

$$
X_{\Pi}(f)=[f, \Pi] .
$$

Soit $Z$ un champ de vecteurs formel s'annullant à l'ordre deux en $0 \in \mathbb{C}^{n}$. On a la relation bien connue

$$
\exp (Z)^{*}(B)=\sum_{i \geq 0} \frac{a d_{Z}^{i} B}{i !},
$$

où on note $a d_{Z}$ l'application linéaire $[Z,$.$] , et \exp (Z)$ le temps 1 du flot formel du champ formel $Z$. Pour plus d'informations sur le crochet de Schouten on renvoie à [6].

Champ hamiltonien. - Pour tout $1 \leq i \leq n$ posons $Y_{i}:=x_{i} \partial_{i}$. Soit $P:=$ $\sum_{i \neq j} p_{i j}(x) Y_{i} \wedge Y_{j}$ un champ de bivecteurs et posons $P_{i}:=\sum_{j=1}^{n} p_{i j} Y_{j}$. Soit $f$ une fonction. On définit le champ « hamiltonien » de $f$ par rapport à $P$ qu'on note $X_{P}(f)$ (on met des guillemets parce que $P$ n'est pas nécessairement une structure de Poisson) par

$$
\begin{aligned}
X_{P}(f) & =\frac{1}{2} \sum_{i \neq j} p_{i j}\left(x_{i} \partial_{i} f Y_{j}-x_{j} \partial_{j} f Y_{i}\right) \\
& =\sum_{i=1}^{n} x_{i} \partial_{i} f P_{i}(x) .
\end{aligned}
$$

Structure de Poisson quadratique diagonale. - Un champ de bivecteurs sur $\mathbb{C}^{n}$ qui s'écrit $\frac{1}{2} \sum_{i \neq j} \alpha_{i j} Y_{i} \wedge Y_{j}$, où $\alpha_{i j}=-\alpha_{j i}$ est appelé diagonal.

REMARQUE. - Tout champ de bivecteurs quadratique diagonal $\Pi$ définit une structure de Poisson i.e. on a $[\Pi, \Pi]=0$. L'expression diagonale est unique à permutation des coordonnées près (cf. [5]) et si une structure de Poisson à 1-jet nul en un point $p$ possède une partie quadratique diagonale, une structure de 
Poisson $C^{2}$-proche possède un 1-jet nul en un point $p^{\prime}$ proche de $p$, ainsi qu'une partie quadratique diagonale.

Écriture de référence. - Appelons écriture de référence d'un champ de bivecteurs ou d'une structure de Poisson formelle $\mathbb{C}^{n}$ une écriture de la forme $\Pi=\frac{1}{2} \sum_{I, i \neq j} x^{I} \alpha_{i j}^{I} Y_{i} \wedge Y_{j}$, où $Y_{i}:=x_{i} \partial_{i}$ et $\alpha_{i j}^{I}=-\alpha_{j i}^{I}$.

REMARQUe. - Le multi-indices $I$ de $x^{I} \alpha_{i j}^{I} Y_{i} \wedge Y_{j}$ a toutes ces composantes positives où nulles, à l'exception éventuelle de la $i$ ou $j$-ème pouvant prendre la valeur -1 (on rappelle $Y_{i}=x_{i} \partial_{i}$ ).

Soit $I=\left(I_{1}, \ldots, I_{n}\right) \in \mathbb{Z}^{n}$ un multi-indice, et $\Pi:=\frac{1}{2} \sum_{i \neq j} a_{i j} Y_{i} \wedge Y_{j}$ une structure de Poisson diagonal. Posons $A_{i}:=\sum_{j=1}^{n} a_{i j} Y_{i}$. On a

$$
X_{\Pi}\left(x^{I}\right)=x^{I} \sum_{i=1}^{n} I_{i} A_{i} .
$$

Le champ hamiltonien d'un monôme $x^{I}$ relatif à une structure de Poisson quadratique diagonale est donc produit de $x^{I}$ par un champ de vecteurs linéaire diagonal.

Soient $\Pi, \Pi^{\prime}$ des structures de Poisson sur $\mathbb{C}^{n}, Z$ un champ de vecteurs linéaire diagonal sur $\mathbb{C}^{n}$ et $J, K \in \mathbb{Z}^{n}$ des multi-indices. On déduit de (2.1)$(2.4)$

$$
\begin{aligned}
& {\left[x^{J}, \Pi\right]=X_{\Pi}\left(x^{J}\right)} \\
& {\left[\Pi, x^{J} Z\right]=Z \wedge X_{\Pi}\left(x^{J}\right)} \\
& {\left[x^{J} \Pi, x^{K} \Pi^{\prime}\right]=2\left(X_{\Pi^{\prime}}\left(x^{J}\right) \wedge \Pi+X_{\Pi}\left(x^{K}\right) \wedge \Pi^{\prime}\right) .}
\end{aligned}
$$

Lemme 2.1. - (i) Soient $I, J \in \mathbb{Z}^{n}$ des multi-indices, $Z$ un champ de vecteurs linéaire diagonal et $\Pi$ une structure de Poisson quadratique diagonale sur $\mathbb{C}^{n}$. Alors il existe une structure de Poisson quadratique diagonale $\Pi^{\prime}$ telle que $\left[x^{I} Z, x^{J} \Pi\right]=x^{I+J} \Pi^{\prime}$.

(ii) Soient $\Pi^{I}$ et $\Pi^{J}$ deux champs de bivecteurs quadratiques diagonaux sur $\mathbb{C}^{n}$. Alors il existe $c_{i j k} \in \mathbb{C}, 1 \leq i, j, k \leq n$, tels que $\left[x^{I} \Pi^{I}, x^{J} \Pi^{J}\right]=$ $x^{I+J} \sum_{1 \leq i<j<k \leq n} c_{i j k} Y_{i} \wedge Y_{j} \wedge Y_{k}$. 
Forme normale de Dufour-Wade, hypothèse $(\mathbf{H})$. — Soit $\Pi$ une structure de Poisson à 1-jet nul en $0 \in \mathbb{C}^{n}$ de partie quadratique $\Pi^{2}$ diagonale. Posons $\Pi^{2}=$ $\frac{1}{2} \sum_{i \neq j} a_{i j} Y_{i} \wedge Y_{j}$ avec $a_{i j}=-a_{j i}$, et $A_{i}:=\sum_{j=1}^{n} a_{i j} Y_{j}$. On dit que $x^{I}$ est un monôme résonnant si $X_{\Pi^{2}}\left(x^{I}\right)=0$. Ceci revient à ce que pour tout $1 \leq i \leq n$ on a $\mathcal{L}_{A_{i}} x^{I}=0$.

Soit $(\mathrm{H})$ l'hypothèse générique qui suit sur la partie quadratique $\Pi^{2}$ (cf. [5]) : tout monôme $x^{I}$ qui vérifie la propriété suivante est résonnant $: I=\left(I_{1}, \ldots, I_{n}\right)$ comporte deux composantes $I_{i}, I_{j}$ égales à -1 et on a $\left(\sum_{l=1}^{n} \frac{1}{x^{I}} \mathcal{L}_{A_{l}}\left(x^{I}\right)\right) \wedge Y_{i} \wedge$ $Y_{j}=0$. Dans [5] est démontré le résultat qui suit.

Proposition 2.1 (Dufour-Wade). - Si $\Pi$ vérifie $(H)$, alors il existe $\Phi$ transformation formelle telle que

$$
\Phi^{*}(\Pi)=\sum_{i \neq j} \sum_{X_{\Pi^{2}}\left(x^{I}\right)=0} x^{I} \alpha_{i j}^{I} Y_{i} \wedge Y_{j},
$$

On dit que $\Phi^{*}(\Pi)$ est sous forme normale de Dufour-Wade.

Développements asymptotiques. - On reproduit de [16] (avec quelques modifications) des définitions de développements asymptotiques sectoriels. Le lecteur pourra également consulter [8]. Soient $S_{1} \subset \mathbb{C}$ le cercle unité et $U \subset S^{1}$ connexe. On désigne aussi par $U$ le secteur de $\mathbb{C}$, de sommet 0 formé des points $z \neq 0$ tels que $\frac{z}{|z|} \in U$ et $|z|<R$ (le rayon n'est pas précisé en général). On dit que le secteur $U$ est ouvert (resp. fermé) si l'arc qui le représente dans $S^{1}$ est ouvert (resp. fermé).

Soient $U$ un secteur ouvert et $f$ une fonction analytique de $U \backslash\{0\}$ dans $\mathbb{C}$. On dit que $f$ admet $\hat{f}:=\sum_{k \geq 0} f_{k} z^{k} \in \mathbb{C}[[z]]$ comme développement asymptotique au sens de Poincaré si pour tout sous-secteur fermé $\bar{V}$ de $U$, et tout $m>0$ il existe un nombre réel positif $C(\bar{V}, m)$ tel que

$$
\sup _{Z \in \bar{V}}|z|^{-m}\left\|f(z)-\sum_{k=0}^{m-1} f_{k} z^{k}\right\|<C(\bar{V}, m) .
$$

Soient $U$ un secteur ouvert et $\Delta$ un ouvert dans $\mathbb{C}^{j}$. On dit qu'une fonction $f$ analytique de $U \backslash\{0\} \times \Delta$ dans $\mathbb{C}^{l}$ admet la série formelle $\hat{f}(z, x)=\sum_{p \geq 0} f_{p}(x) z^{p}$ où les fonctions $f_{p}$ sont analytiques de $\Delta$ dans $\mathbb{C}^{l}$ comme développement asymptotique (fort) si, pour tout sous-secteur fermé $\bar{V} \subset U$, tout compact $K \subset \Delta$ et tout entier $m>0$,

$$
\sup _{\substack{z \in \bar{V} \\ y \in K}}|z|^{-m}\left\|f(z, x)-\sum_{k=0}^{m-1} f_{k}(x) z^{k}\right\|<C(\bar{V}, K, m) .
$$


Soit $\hat{f} \in \mathbb{C}\left[\left[z, x_{1}, \ldots, x_{n}\right]\right]$ une série formelle qui s'écrit $\hat{f}(z, x)=\sum_{Q \in \mathbb{N}^{n}} \hat{f}_{Q}(z) x^{Q}$. On dit qu'une fonction $f$ analytique sur $U \times \Delta$ (où $U \subset \mathbb{C}$ est un secteur ouvert; $\Delta \in \mathbb{C}^{n}$ un polydisque ouvert de centre $\left.0 \in \mathbb{C}^{n}\right)$ qui s'écrit $f(z, x)=$ $\sum_{Q \in \mathbb{N}^{n}} f_{Q}(z) x^{Q}$ admet $\hat{f}$ comme développement asymptotique au sens de GérardSibuya das $U \times \Delta$, si chacune des fonctions $f_{Q}(z)$ admet $\hat{f}_{Q}(z)$ comme développement asymptotique au sens de Poincaré dans le secteur $U$. Ceci signifie que pour tout sous-secteur fermé $U^{\prime} \subset U$ tout compact $K \subset U$, et pour tout entier $k$, il existe une constante $C\left(k, U^{\prime}, K\right)$ telle que

$$
\left|f(z, x)-f^{k}(z, x)\right| \leq C\left(k, U^{\prime}, K\|(z, x)\|^{k+1}\right.
$$

pour tout $(z, x) \in U^{\prime} \times K$, où $f^{k}$ désigne le $k$-jet de $f$ en $0 \in \mathbb{C}^{n+1}$ et $\|$. désigne une norme quelconque sur $\mathbb{C}^{n+1}$.

Soit $R=\left(R_{1}, \ldots, R_{n}\right) \in \mathbb{N}^{n}$ un multiindice et $\pi$ l'application $\mathbb{C}^{n} \rightarrow \mathbb{C}$ qui à $\left(x_{1}, \ldots, x_{n}\right)$ associe $x^{R_{1}} \cdots x^{R_{n}}$. On dit qu'une fonction analytique sur $\pi^{-1}(U) \cap \Delta$ (où $U \subset \mathbb{C}$ est un secteur ouvert; $\Delta \subset \mathbb{C}^{n}$ est un polydisque ouvert de centre $\left.0 \in \mathbb{C}^{n}\right)$, qui s'écrit

$$
f(z, x)=\sum_{Q \in \mathbb{N}^{n}} f_{Q}\left(x^{R}\right) x^{Q}
$$

admet $\hat{f}=\sum_{Q \in \mathbb{N}^{n}} \hat{f}_{Q}\left(x^{R}\right) x^{Q}$ comme développement asymptotique au sens de Gérard-Sibuya en le monôme $x^{R}$ dans $\pi^{-1}(U) \cap \Delta$, si chacune des fonctions $f_{Q}(z)$ admet $\hat{f}_{Q}(z)$ comme développement asymptotique au sens de Poincaré, dans le secteur $U$.

\section{Théorème de normalisation sectorielle}

Soit $\Pi^{2}:=\frac{1}{2} \sum_{i \neq j} a_{i j} Y_{i} \wedge Y_{j}$ avec $a_{i j}=-a_{j i}$ une structure de Poisson quadratique diagonale sur $\mathbb{C}^{n}$. Posons $A_{i}:=\sum_{j=1}^{n} a_{i j} Y_{j}$ et soit $\beta_{i}^{I} \in \mathbb{C}$ tel que $\mathcal{L}_{A_{i}} x^{I}=\beta_{i} x^{I}$. On dit que $\Pi^{2}$ satisfait la condition diophantienne de petits diviseurs (D) si

$$
-\sum_{k \geq 0} \frac{\ln \omega_{k}}{2^{k}}<+\infty,
$$

avec $\omega_{k}:=\inf \left\{\max _{1 \leq i \leq n}\left|\beta_{i}^{I}\right| ; \max _{1 \leq i \leq n}\left|\beta_{i}^{I}\right| \neq 0,|I| \leq 2^{k}\right\}$. Pour plus d'information sur la condition (D) on renvoie le lecteur à [9], [17], [19]. Soit $R \in \mathbb{N}^{n}$ et désignions par $D S_{j}^{R}\left(r, r^{\prime}, \delta\right), j=0,1$, les domaines « sectoriels » suivantes :

$D S_{j}^{R}\left(r, r^{\prime}, \delta\right)=\left\{y \in \mathbb{C}^{n}|| \arg y^{R}-\left(\arg \delta+\pi\left(j+\frac{1}{2}\right)\right)|<\pi-\varepsilon, 0<| y^{R}|<r,| y_{i} \mid<r^{\prime}\right\}$, 
avec $r, r^{\prime}>0$ et $\delta \in \mathbb{C}^{*}$.

On considère des structure de Poisson $\Pi$ holomorphes au voisinage de $0 \in \mathbb{C}^{n}$ à 1-jet nul dont la partie quadratique $\Pi^{2}$ de vérifie l'hypothèse $(\mathrm{H})$ de DufourWade (qui assure l'existence d'une forme normale de DufourWade). De plus, on demande les propriétés suivantes satisfaites :

(P1) $\Pi^{2}$ est 1-résonnante : les monômes résonnants $x^{I}$ (i.e. tels que $\left[\Pi^{2}, x^{I}\right]=$ 0 ) sont tous puissances d'un monôme $x^{R}$, appellé «générateur de résonances ».

(P2) La partie quadratique $\Pi^{2}$ est produit extérieur de deux champs de vecteurs linéaires diagonaux $S_{1}$ et $S_{2}$.

(P3) La partie quadratique $\Pi^{2}$ satisfait la condition diophantienne (D).

(P4) Le terme résonnant de plus petit degré est de la forme $x^{R} \Pi^{R}$, où $\Pi^{R}=$ $S \wedge C$, avec $S \in \operatorname{Vect}\left(S_{1}, S_{2}\right)$, et $C$ champ de vecteurs linéaire diagonal vérifiant $\mathcal{L}_{C}\left(x^{R}\right) \neq 0$.

(P5) Toutes les coordonnées de $R$ sont strictement positives.

(P6) Si on note $S_{1}=s_{1} Y_{1}+\cdots+s_{n} Y_{n}$, et $S_{2}=s_{1}^{\prime} Y_{1}+\cdots+s^{\prime} n Y_{n}$, alors supposons les coefficients $s_{i}, s_{i}^{\prime}$ tous situés sur une même droite du plan complexe passant par l'origine.

(P7) Supposons qu'il existe $\Lambda_{0}:=\left(\lambda_{1}, \ldots, \lambda_{n}\right) \in \mathbb{C}^{n}$ tel que pour $\left(\mu_{i}\right)_{1 \leq i \leq n}$, $\left(\alpha_{i}\right)_{1 \leq i \leq n}$ vérifiant $\frac{1}{x^{\Lambda_{0}}} X_{\left[\Pi^{2}+x^{R} \Pi^{R}\right]}\left(x^{\Lambda_{0}}\right):=\sum_{i=1}^{n} x_{i}\left(\mu_{i}+\alpha_{i} x^{R}\right) \partial_{i}$ il existe un indice $i_{0}$ avec $\mu_{i_{0}} \neq 0$ et

$$
\min _{i \neq i_{0}} \operatorname{Re}\left(\frac{\alpha_{i}}{\beta}-\frac{\mu_{i}}{\mu_{i_{0}}} \frac{\alpha_{i_{0}}}{\beta}\right)>0 .
$$

(Le champ $\frac{1}{x^{\Lambda}} X_{\left[\Pi^{2}+x^{R} \Pi^{R}\right]}\left(x^{\Lambda}\right)$ ne dépend pas de la détermination de $x^{\Lambda}$ choisie)

Sous hypothèse $(\mathrm{P} 6)$ il existe $\delta \in \mathbb{C},|\delta|=1$ tel que le nombres $\frac{\mu_{i}}{\delta\left(\sum_{j=1}^{n} R_{j} \alpha_{j}\right)}$ soient tous réels.

THÉORÈme 3.1 (de normalisation sectorielle). - Soit П vérifiant (P1)-(P7). Il existe un ensemble $\mathfrak{A} \in \mathbb{C}^{n}$ intersection d'un ouvert avec un ensemble de mesure de Lebesque pleine et ne dépendant que de $\Pi^{2}$ et de $\Pi^{R}$, tel que pour $\Lambda \in \mathfrak{A}$ on ait les propriétés suivantes : il existe une unité analytique $v_{\Lambda}$ (unique), $r, r^{\prime}>0$ et des transformations holomorphes $\Phi_{j}^{\Lambda}, j=0,1$, (uniques) définies sur les domaines sectoriels $D S_{j}^{R}\left(r, r^{\prime}, \delta\right)$, telles que

(i) les transformations $\Phi_{j}^{\Lambda}$ conjuguent $v_{\Lambda} \Pi$ (qui est une structure de Poisson) à la forme normale polynomiale $\Pi^{2}+x^{R} \Pi^{R}$ sur les domaines sectoriels $D S_{j}^{R}\left(r, r^{\prime}, \delta\right), j=0,1$; 
(ii) on $a x^{\Lambda} \circ \Phi_{j}^{\Lambda}=x^{\Lambda}, x^{R} \circ \phi_{j}^{\Lambda}=x^{R}$.

Les transformations $\Phi_{j}^{\Lambda} j=0,1$, admettent $\hat{\Phi}$ comme développement asymptotique au sens de Gérard-Sibuya en le monôme $x^{R}$ dans $D S_{j}\left(r, r^{\prime}, \delta\right)$, où $\hat{\Phi}$ est l'unique série formelle telle que $(\hat{\Phi})^{*}\left(v_{\Lambda} \Pi\right)=\Pi^{2}+x^{R} \Pi^{R}$ et telle qu'on a $x^{\Lambda} \circ \hat{\Phi}=x^{\Lambda}, x^{R} \circ \hat{\phi}=x^{R}$.

\section{Forme normale formelle polynomiale}

Proposition 4.1 (de normalisation formelle). - Soit $\Pi$ une structure de Poisson à 1-jet nul. Supposons les hypothèses (P1) et (P4) (cf. section 3) satisfaites, ainsi que l'hypothèse $(H)$ de Dufour-Wade. Alors il existe $\widehat{\Phi}$ transformation formelle telle que $\widehat{\Phi}^{*}(\Pi)=\Pi^{2}+x^{R} \Pi^{R}$.

Remarque. - Dans le cas où $R$ comporte des composantes égales à -1 , la forme normale possède au plus un terme résonnant, et la normalisibilité formelle à la forme normale polynômiale $\Pi^{2}+x^{R} \Pi^{R}$ est conséquence directe de la proposition 2.1.

Preuve de la proposition 4.1. - Montrons la proposition par récurrence sur le degré de la normalisation $d$. Supposons $\Pi$ normalisé au degré $d$. Plus précisément, supposons

$$
\Pi:=\Pi^{2}+x^{R} \Pi^{R}+\sum_{|I| \leq d+1} x^{I} \Pi^{I}+\left(x^{(j+1) R}\right) \Pi^{(j+1) R}+\mathfrak{R}^{d+2},
$$

où on note $x^{I}$ les monômes non-résonnants, et $\left(x^{(j+1) R}\right) \Pi^{(j+1) R}$ le terme résonnant (éventuellement nul) de degré $d+1+|R|$. Par $\mathfrak{R}^{d+2}$ on désigne la somme des termes d'ordre supérieur à $d+1$ (pour les termes non-résonnants) et d'ordre supérieur à $d+1+|R|$ pour les termes résonnants.

Il s'agit de montrer qu'il existe une transformation formelle normalisant $\Pi$ au degré $d+1$.

D'après la relation $(2.5)$ et le lemme 2.1 ceci revient à trouver un champ de vecteurs $Z=\sum_{|J|=d+1} x^{J} Z^{J}$, avec $Z^{J}$ linéaire diagonal, tel que

(i) pour $J$ non résonnant, la relation $\left[\Pi^{2}, x^{J} Z^{J}\right]=x^{J} \Pi^{J}$ soit satisfaite,

(ii) et pour $J$ résonnant de degré $d$ vérifiant $J=j R$, la relation

$$
\begin{aligned}
{\left[\Pi^{2}+x^{R} \Pi^{R}, x^{j R} Z^{j R}\right] } & =\left[x^{R} \Pi^{R}, x^{j R} Z^{j R}\right] \\
& =x^{(j+1) R} \Pi^{(j+1) R}
\end{aligned}
$$

soit satisfaite. 
Rappelons que les multiindices $J$ peuvent avoir une composante égale à -1 , dans le cas où $Z$ reste analytique en 0 . Pour le cas (i) (non-résonnant) on renvoie le lecteur à la démonstration de la proposition 2.1 dans [5] - on se limite donc à montrer que l'équation (4.1) admet une solution.

Pour $\Pi$ normalisé au degré $d$, l'identité de Jacobi $[\Pi, \Pi]=0$ implique que le terme résonnant de degré $d+1+|R|$, qu'on note $x^{(j+1) R} \Pi^{(j+1) R}$, vérifie

$$
\left[x^{R} \Pi^{R}, x^{(j+1) R} \Pi^{(j+1) R}\right]=0 .
$$

La proposition 4.1 est donc une conséquence de la proposition 4.2 qui suit.

Proposition 4.2. - Pour tout champ de bivecteurs quadratique diagonal $\Pi^{(j+1) R}$ vérifiant (4.2), il existe $Z$ champ de vecteurs linéaire diagonal qui satisfait (4.1).

Notations. - Pour la preuve de la proposition 4.2, on note $\Pi^{2}:=\frac{1}{2} \sum_{i \neq j} a_{i j} Y_{i} \wedge$ $Y_{j}$ avec $a_{i j}$ tel que $a_{i j}=-a_{j i}$. On définit le $i$-ème champ de vecteurs linéaire diagonal « ligne » $A_{i}:=\sum_{j=1}^{n} a_{i j} Y_{j}$. Notons $\mathbb{S}:=\left\langle A_{1}, \ldots, A_{n}\right\rangle=\left\langle S_{1}, S_{2}\right\rangle$ l'algèbre de Lie abélienne engendrée par $S_{i}, i=1,2$ (cf. l'hypothèse (P2) et le théorème 3.1).

Pour la preuve de la proposition 4.2 on a besoin des lemmes suivants :

LEMME 4.1. - Soit $\Pi^{\prime}$ champ bivecteur quadratique diagonal tel que $X_{\Pi^{R}}\left(x^{j R}\right) \wedge \Pi^{\prime}=0$. Alors il existe $Z$ champ de vecteurs linéaire diagonal tel que $\left[x^{j R} Z, \Pi^{R}\right]=x^{j R} \Pi^{\prime}$. De plus, $x^{j R} \Pi^{\prime}=X_{\Pi^{R}}\left(x^{j R}\right) \wedge Z$.

Preuve du lemme 4.1. - On rappelle que $R$ n'a aucune composante strictement négative. Il est facile de voir que $X_{\Pi^{R}}\left(x^{j R}\right) \neq 0$. La première conclusion du lemme 4.1 est un rappel de la proposition 3 dans [5]. La relation $x^{j R} \Pi^{\prime}=X_{\Pi^{R}}\left(x^{j R}\right) \wedge Z$ vient de $(2.9)$.

LEMME 4.2. - Soit $x^{j R}$ un monôme « résonnant». Alors

(i) Il existe $c \in \mathbb{C}$ tel que $x^{j R} \Pi^{R}=X_{\Pi^{R}}\left(x^{j R}\right) \wedge c C$. D'après (2.9) ceci signifie $\left[c x^{j R} C, \Pi^{R}\right]=x^{j R} \Pi^{R}$.

(ii) $\mathcal{L}_{\left[X_{\Pi_{R}}\left(x^{j R}\right)\right]}\left(x^{j R}\right)=0$ et $\mathcal{L}_{c C}\left(x^{j R}\right)=-1$ (On rappelle que $X_{\Pi^{R}}\left(x^{j R}\right)$ désigne le champ hamiltonien de $x^{j R}$ relatif $\left.\grave{a} \Pi^{R}\right)$.

(iii) Tout champ de bivecteurs $B=\left[x^{j R} X, x^{R} \Pi^{R}\right]$ avec $X$ champ de vecteurs linéaire diagonal satisfait $\left[x^{R} \Pi^{R}, B\right]=0$.

(iv) Tout champ de bivecteurs $B=x^{R}\left[x^{j R} X, \Pi^{R}\right]$ avec $X$ champ de vecteurs linéaire diagonal satisfait $B=\left[x^{j R} X^{\prime}, x^{R} \Pi^{R}\right]$, où $X^{\prime}$ champ de vecteurs linéaire diagonal. 
Preuve du lemme 4.2. - Par hypothèse, $\Pi^{R}:=S \wedge C$, où $S \in \mathbb{S}$ et $C$ diagonal tel que $\mathcal{L}_{C}\left(x^{R}\right) \neq 0$ (Hypothèse (P4) section 3). On en déduit

$$
X_{\Pi^{R}}\left(x^{j R}\right)=\mathcal{L}_{S}\left(x^{j R}\right) C-\mathcal{L}_{C}\left(x^{j R}\right) S .
$$

Par $\mathcal{L}_{S}\left(x^{j R}\right)=0$ on obtient $X_{\Pi^{R}}\left(x^{j R}\right)=-\mathcal{L}_{C}\left(x^{j R}\right) S$. On obtient le point (i) du lemme 4.2 en posant $c:=-\frac{1}{j R . C}$, où $R . C:=\sum_{i+1}^{n} R_{i} c_{i}$ avec $R:=$ $\left(R_{1}, \ldots, R_{n}\right), C:=\left(c_{1} Y_{i}, \ldots, c_{n} Y_{n}\right)$.

L'égalité $\mathcal{L}_{\left[X_{\Pi R}\left(x^{j R}\right)\right]}\left(x^{j R}\right)=0$ n'est rien d'autre que le fait bien connu que le champ hamiltonien d'une fonction préserve la fonction.

Les relations $x^{j R} \Pi^{R}=X_{\Pi^{R}}\left(x^{j R}\right) \wedge c C$ et

$$
X_{\left[x^{j R} \Pi^{R}\right]}\left(x^{j R}\right)=\mathcal{L}_{\left[X_{\Pi^{R}}\left(x^{j R}\right)\right]}\left(x^{j R}\right) c C-\mathcal{L}_{c C}\left(x^{j R}\right) X_{\Pi^{R}}\left(x^{j R}\right)
$$

impliquent $\mathcal{L}_{c C}\left(x^{j R}\right)=-1$, c'est-à-dire le point (ii) du lemme 4.2.

Soit $\Pi_{n}:=\Pi^{2}+x^{R} \Pi^{R}$. On vérifie que $\Pi_{n}$ est une structure de Poisson, i.e. $\left[\Pi_{n}, \Pi_{n}\right]=0$. On obtient alors le point (iii) du lemme 4.2 à partir de $\left[\left(I d+x^{j R} X\right)^{*}\left(\Pi_{n}\right),\left(I d+x^{j R}\right)^{*} \Pi_{n}\right]=0$ via les relations (2.9)-(2.10).

Reste à montrer le point (iv) du lemme 4.2. D'après (2.2),

$$
B=\left[x^{j R} X, x^{R} \Pi^{R}\right]=x^{R}\left[x^{j R} X, \Pi^{R}\right]+x^{j R} \mathcal{L}_{X}\left(x^{R}\right) \Pi^{R} .
$$

Montrons qu'en ajoutant à $X$ un multiple approprié de $C$ permettant de compenser $x^{j R} \mathcal{L}_{X}\left(x^{R}\right) \Pi^{R}$ dans (4.3), on obtient $X^{\prime}$ tel que $B=\left[x^{j R} X^{\prime}, x^{R} \Pi^{R}\right]$. D'après $(2.9)$,

$$
\left[x^{j R} C, x^{R} \Pi^{R}\right]=x^{R} X_{\Pi^{R}}\left(x^{j R}\right) \wedge C+x^{j R} \mathcal{L}_{C}\left(x^{R}\right) \Pi^{R} .
$$

Par les relations $X_{\Pi^{R}}\left(x^{j R}\right)=\mathcal{L}_{S}\left(x^{j R}\right) C-\mathcal{L}_{C}\left(x^{j R}\right) S$ et $\mathcal{L}_{S}\left(x^{j R}\right)=0$, le champ de bivecteurs $x^{R} X_{\Pi^{R}}\left(x^{j R}\right) \wedge C$ est nul.

Preuve de la proposition 4.2. - Il s'agit de montrer l'existence de $Z^{\prime}{ }^{j}$ champ de vecteurs linéaire diagonal tel que $\left[x^{j R} Z^{\prime}, \Pi^{R}\right]=x^{j R} \Pi^{(j+1) R}$. Par le point (iv) du lemme 4.2 il suffit de montrer la proposition 4.3 qui suit.

Proposition 4.3. - Supposons $\left[x^{R} \Pi^{R}, x^{(j+1) R} \Pi^{(j+1) R}\right]=0$. Alors il existe $Z^{\prime j}$ champ de vecteurs linéaire diagonal tel que $\left[x^{j R} Z^{\prime j}, \Pi^{R}\right]=x^{j R} \Pi^{(j+1) R}$.

Preuve de la proposition 4.3. - Par le lemme 4.1, il suffit de montrer $X_{\Pi^{R}}\left(x^{j R}\right) \wedge \Pi^{(j+1) R}=0$. Or, les champs hamiltoniens $X_{\Pi^{R}}\left(x^{j R}\right)$ et $X_{\Pi^{R}}\left(x^{(j+1) R}\right)$ sont parallels. En effet, par $\Pi^{R}=S \wedge R$ on obtient

$$
\begin{gathered}
X_{\Pi^{R}}\left(x^{j R}\right)=\mathcal{L}_{S}\left(x^{j R}\right) C-\mathcal{L}_{C}\left(x^{j R}\right) S, \\
X_{\Pi^{R}}\left(x^{(j+1) R}\right)=\mathcal{L}_{S}\left(x^{(j+1) R}\right) C-\mathcal{L}_{C}\left(x^{(j+1) R}\right) S .
\end{gathered}
$$

Avec $\mathcal{L}_{S}\left(x^{j R}\right)=0$ et $\mathcal{L}_{S}\left(x^{(j+1) R}\right)=0$ on obtient

$$
X_{\Pi^{R}}\left(x^{j R}\right)=-\mathcal{L}_{C}\left(x^{j R}\right) S, \quad X_{\Pi^{R}}\left(x^{(j+1) R}\right)=-\mathcal{L}_{C}\left(x^{(j+1) R}\right) S .
$$


Pour prouver la proposition 4.3 il suffit donc de montrer

$$
X_{\Pi^{R}}\left(x^{(j+1) R}\right) \wedge \Pi^{(j+1) R}=0 .
$$

Faisons l'abus de langage suivant : soit $V=v_{1} Y_{i}+\cdots+v_{n} Y_{n}$ un champ de vecteur linéaire diagonal. Associons à $V$ le vecteur $\left(v_{1}, \ldots, v_{n}\right) \in \mathbb{C}^{n}$. On dit que deux champs de vecteurs linéaires diagonaux ainsi que leurs produits par des fonctions sont orthogonaux si leurs vecteurs respectivement associés le sont au sens du produit scalaire standard.

D'après (2.10), l'identité de Jacobi $[\Pi, \Pi]=0$ donne, en identifiant les termes en $x^{(j+2) R}$,

$$
\begin{aligned}
0 & =\left[x^{R} \Pi^{R}, x^{(j+1) R} \Pi^{(j+1) R}\right] \\
& =2\left(X_{\left[x^{R} \Pi^{R}\right]}\left(x^{(j+1) R}\right) \wedge \Pi^{(j+1) R}+X_{\left[x^{(j+1) R} \Pi^{(j+1) R}\right]}\left(x^{R}\right) \wedge \Pi^{R}\right) .
\end{aligned}
$$

D'après le point (i) du lemme 4.2,

$$
\Pi^{R}=\frac{1}{x^{(j+1) R}} X_{\Pi^{R}}\left(x^{(j+1) R}\right) \wedge c C .
$$

$\operatorname{Par}(4.5) X_{\Pi^{R}}\left(x^{(j+1) R}\right) \wedge \Pi^{(j+1) R}$ est donc un produit extérieur de trois champs de vecteurs et on peut écrire

$$
X_{\Pi^{R}}\left(x^{(j+1) R}\right) \wedge \Pi^{(j+1) R}=X_{\Pi^{R}}\left(x^{(j+1) R}\right) \wedge A \wedge B
$$

avec $A, B$ champs diagonaux « orthogonaux » à $X_{\Pi^{R}}\left(x^{(j+1) R}\right)$. On peut même supposer $B=c C$. Alors on a $\Pi^{(j+1) R}=A \wedge c C+\Pi^{\prime(j+1) R}, \operatorname{avec} X_{\Pi^{R}}\left(x^{(j+1) R}\right) \wedge$ $\Pi^{\prime(j+1) R}=0$.

Pour montrer (4.4), on peut donc dorénavant supposer que $\Pi^{(j+1) R}=A \wedge c C$. Terminons en montrant que $A$ est nul. La relation (4.6) devient

$$
X_{x^{R} \Pi^{R}}\left(x^{(j+1) R}\right) \wedge A \wedge c C=-X_{A \wedge c C}\left(x^{R}\right) \wedge X_{\Pi^{R}}\left(x^{(j+1) R}\right) \wedge c C .
$$

Développons le membre gauche de (4.7). Avec les relations

$$
X_{A \wedge c C}\left(x^{R}\right)=\mathcal{L}_{A}\left(x^{R}\right) c C-\mathcal{L}_{c C}\left(x^{R}\right) A, \quad \Pi^{R}=\frac{1}{x^{(j+1) R}} X_{\Pi^{R}}\left(x^{(j+1) R}\right) \wedge c C,
$$

on obtient

$$
X_{\Pi^{R}}\left(x^{(j+1) R}\right)=\frac{1}{x^{(j+1) R}}\left[\mathcal{L}_{\left[X_{\Pi^{R}}\left(x^{(j+1) R}\right)\right]}\left(x^{(j+1) R}\right) c C-\mathcal{L}_{c C}\left(x^{(j+1) R}\right) X_{\Pi^{R}}\left(x^{(j+1) R}\right)\right] .
$$

Or on a $\mathcal{L}_{\left[X_{\Pi} R\left(x^{(j+1) R}\right)\right]}\left(x^{(j+1) R}\right)=0$ en tant que dérivée de Lie de $x^{(j+1) R}$ le long d'un champ hamiltonien de $x^{(j+1) R}$. La relation (4.7) devient

$$
\begin{aligned}
& -\frac{1}{x^{(j+1) R}} \mathcal{L}_{c C}\left(x^{(j+1) R}\right) X_{x^{R} \Pi^{R}}\left(x^{(j+1) R}\right) \wedge A \wedge c C=\mathcal{L}_{c C}\left(x^{R}\right) A \wedge X_{\Pi^{R}}\left(x^{(j+1) R}\right) \wedge c C . \\
& \quad \text { томе } 137-2009-\text { N }^{\mathrm{o}} 3
\end{aligned}
$$


Mais (4.8) a lieu uniquement dans le cas où $A=0$, ou bien dans le cas où $\frac{1}{x^{(j+1) R}} \mathcal{L}_{c C}\left(x^{(j+1) R}\right)=\frac{1}{x^{R}} \mathcal{L}_{c C}\left(x^{R}\right)$. Comme le dernier cas est exclu pour $j \geq 1$, on a nécessairement $A=0$.

On aura ultérieurement besoin du lemme 4.3 qui s'inscrit dans ce qui précède.

LEMme 4.3. - Un champ de vecteurs de la forme $x^{j R} Z, Z$ linéaire diagonal, tel que $\left[x^{j R} Z, x^{R} \Pi^{R}\right]=x^{(j+1) R} \Pi^{(j+1) R}$ est unique à addition d'un multiple $d u$ champ hamiltonien $X_{\Pi^{R}}\left(x^{J}\right)$ près.

Preuve du lemme 4.3. - Soient $Z_{1}, Z_{2}$ tel que

$$
\left[x^{j R} Z_{1}, x^{R} \Pi^{R}\right]=\left[x^{j R} Z_{2}, x^{R} \Pi^{R}\right]=x^{(j+1) R} \Pi^{(j+1) R} .
$$

Posons $Z:=Z_{1}-Z_{2}$. On a $\left[x^{j R} Z, x^{R} \Pi^{R}\right]=0$. D'après $(2.9)$ et $\mathcal{L}_{S}\left(x^{R}\right)=0$,

$$
-x^{j R} \mathcal{L}_{Z}\left(x^{R}\right) \Pi^{R}=x^{R} X_{\Pi^{R}}\left(x^{j R}\right) \wedge Z=-x^{R} \mathcal{L}_{C}\left(x^{j R}\right) S \wedge Z .
$$

Comme $\Pi^{R}=S \wedge C, Z$ est nécessairement de la forme $Z=\lambda C+\mu S$, où $\lambda, \mu \in \mathbb{C}$. Par $\mathcal{L}_{S}\left(x^{R}\right)=0$ suit la relation $\mathcal{L}_{\mu C}\left(x^{R}\right)=\mu \mathcal{L}_{C}\left(x^{j R}\right)$, qui implique $\mu=0$ pour $j>1$. Donc $Z$ est multiple de $S$, et le lemme 4.3 suit de $X_{\Pi^{R}}\left(x^{j R}\right)=$ $-\mathcal{L}_{C}\left(x^{j R}\right) S$.

\section{Exemple d'une structure de Poisson à 1-jet nul, forme normale polynomiale, tel que toute transformation formelle normalisante diverge}

Donnons un exemple de structure de Poisson $\Pi$ à 1-jet nul en $0 \in \mathbb{C}^{n}$ de partie quadratique 1-résonnante et admettant une forme normale polynomiale. On veut également que toute transformation conjuguant $\Pi$ à la forme normale polynomiale diverge.

Soient $S$ un champ de vecteurs linéaire diagonal 1-résonnant de $\mathbb{C}^{n}$ et $x^{R}$ son générateur de résonances, et supposons toutes les coordonnées de $R$ strictement positives. Soit $S_{1}$ un champ de vecteur linéaire diagonal tel que $\mathcal{L}_{S_{1}}\left(x^{R}\right)=0$, et tel que la première coordonnée de $S_{1}$ soit nulle. On a alors $\mathcal{L}_{S_{1}}\left(x_{1}\right)=0$. Soit $C$ un champ de vecteurs linéaire diagonal tel que $\mathcal{L}_{C}\left(x^{R}\right) \neq 0$.

Posons $\Pi^{2}=S \wedge S_{1}, \Pi^{R}=S_{1} \wedge C$ et considérons le champ de bivecteurs

$$
\Pi:=\Pi^{2}+x^{R} \Pi^{R}+x_{1}^{l_{0}} \Pi^{R} .
$$

Lemme 5.1. - $\Pi$ définit une structure de Poisson. 
Démonstration. - Il s'agit d'établir $[\Pi, \Pi]=0$. Par bilinéarité et (2.1),

$$
\begin{aligned}
{[\Pi, \Pi] } & =\left[\Pi^{2}, \Pi^{2}\right]+2\left[\Pi^{2}, x^{R} \Pi^{R}\right]+\left[x^{R} \Pi^{R}, x^{R} \Pi^{R}\right] \\
& +2\left[\Pi^{2}, x_{1}^{l_{0}} \Pi^{R}\right]+2\left[x^{R} \Pi^{R}, x_{1}^{l_{0}} \Pi^{R}\right]+\left[x_{1}^{l_{0}} \Pi^{R}, x_{1}^{l_{0}} \Pi^{R}\right]
\end{aligned}
$$

Montrons que tous les crochets du deuxième membre sont nuls. On a $\left[\Pi^{2}, \Pi^{2}\right]=$ 0 (comme pour tout champ de bivecteurs quadratique diagonal). De plus, comme $x^{R}$ est résonnant, on a d'après (2.7), (2.10), la relation $\left[\Pi^{2}, x^{R} \Pi^{R}\right]=0$.

D'après la relation (2.10), $\left[x^{R} \Pi^{R}, x^{R} \Pi^{R}\right]=2 x^{R} X_{\Pi^{R}}\left(x^{R}\right) \wedge \Pi^{R}$. De $\Pi^{R}=$ $S_{1} \wedge C$ suit

$$
X_{\Pi^{R}}\left(x^{R}\right)=\mathcal{L}_{S_{1}}\left(x^{R}\right) C-\mathcal{L}_{C}\left(x^{R}\right) S_{1}=-\mathcal{L}_{C}\left(x^{R}\right) S_{1} \Pi^{R}=0,
$$

puisque $x^{R}$ est résonnant, c'est-à-dire $\mathcal{L}_{S_{1}}\left(x^{R}\right)=0$.

D'après $(2.10)$,

$$
\begin{aligned}
{\left[x^{R} \Pi^{R},\left(x_{1}\right)^{l_{0}} \Pi^{R}\right] } & =x^{R} X_{\Pi^{R}}\left(\left(x_{1}\right)^{l_{0}}\right) \wedge \Pi^{R}+\left(x_{1}\right)^{l_{0}} X_{\Pi^{R}}\left(x^{R}\right) \wedge \Pi^{R} \\
& =x^{R} X_{\Pi^{R}}\left(\left(x_{1}\right)^{l_{0}}\right) \wedge \Pi^{R}
\end{aligned}
$$

car d'après le point précédent, $X_{\Pi^{R}}\left(x^{R}\right) \wedge \Pi^{R}$ est nul. Par définition

$$
X_{\Pi^{R}}\left(x_{1}\right)^{l_{0}}=\mathcal{L}_{S_{1}}\left(\left(x_{1}\right)^{l_{0}}\right) C-\mathcal{L}_{C}\left(\left(x_{1}\right)^{l_{0}}\right) S_{1} .
$$

Or, comme la première coordonnée de $S_{1}$ est nulle, $\mathcal{L}_{S_{1}}\left(\left(x_{1}\right)^{l_{0}}\right)=0$. De $\mathcal{L}_{C}\left(\left(x_{1}\right)^{l_{0}}\right) S_{1} \wedge \Pi^{R}=0$ suit $\left[x^{R} \Pi^{R},\left(x_{1}\right)^{l_{0}} \Pi^{R}\right]=0$.

En remplaçant $x^{R}$ par $x_{1}^{l_{0}}$ on obtient de même que $\left[\left(x_{1}\right)^{l_{0}} \Pi^{R},\left(x_{1}\right)^{l_{0}} \Pi^{R}\right]=$ 0 .

D'après la proposition 4.1, $\Pi$ admet $\Pi^{2}+x^{R} \Pi^{R}$ comme forme normale formelle.

Proposition 5.1. - Toute transformation formelle $\widehat{\Psi}$ conjuguant $\Pi$ à sa forme normale $\Pi^{2}+x^{R} \Pi^{R}$ diverge.

Normalisation formelle. - Soit $\widehat{\Psi}$ une transformation formelle. On pourra poser $\widehat{\Psi}=\cdots \circ \exp \left(Z_{d}\right) \circ \cdots \circ \exp \left(Z_{l}\right)$, tel que pour tout $d, Z_{d}$ est homogène de degré $d+1$ i.e. $Z_{d}$ s'écrit comme produit d'un monôme de degré $d$ (on rappelle que l'exposant peut éventuellement avoir une composante égale à -1), et d'un champ de vecteurs linéaire diagonal.

Supposons que $\psi^{d-1}:=\exp \left(Z_{d-1}\right) \circ \cdots \circ \exp \left(Z_{l}\right)$ normalise $\Pi$ au degré $d$, c'est-à-dire

$$
\left(\psi^{d-1}\right)^{*}(\Pi):=\Pi^{2}+x^{R} \Pi^{R}+\sum_{|I| \leq d} x^{I} \Pi^{I}+\left(x^{(j+1) R}\right) \Pi^{(j+1) R}+\mathfrak{R}^{d+1}
$$

où on note $x^{I}$ les monômes non-résonnants, et $\left(x^{(j+1) R}\right) \Pi^{(j+1) R}$ les termes résonnants (éventuellement nuls de degré $d+|R|$. La notation $\mathfrak{R}^{d+2}$ désigne les termes d'ordre supérieur à $d(\operatorname{resp} d+|R|)$.

TOME $137-2009-\mathrm{N}^{\mathrm{O}} 3$ 
Posons $Z_{d}:=\sum_{|J|=d} x^{J} Z^{J}$, avec $Z^{J}$ champ de vecteurs linéaire diagonal. On a vu dans la preuve de la proposition 4.1 , pour que $\psi^{d}:=\exp \left(Z_{d}\right) \circ \cdots \circ \exp \left(Z_{l}\right)$ normalise $\Pi$ au degré $d+1$, il faut et il suffit que

$$
\left[\Pi^{2}, x^{I} Z^{I}\right]=x^{I} \Pi^{I},
$$

pour $x^{I}$ non-résonnant, et pour le terme résonnant $x^{j R} Z^{j R}$ de $Z_{d}$, il faut et il suffit que

$$
\left[x^{R} \Pi^{R}, x^{j R} Z^{j R}\right]=x^{(j+1) R} \Pi^{(j+1) R} .
$$

D'après (2.9), dans le cas non-résonnant, les termes $x^{J} Z^{J}$ sont uniques à addition d'un multiple du champ hamiltonien $X_{\Pi^{2}}\left(x^{J}\right)$ près.

On note $\mathcal{F}$ l'ensemble des champs de bivecteurs formels s'écrivant sous la forme

$$
\left(\sum_{l, m} c_{l m}\left(x^{R}\right)^{m}\left(x_{1}\right)^{l}\right) \Pi^{R},
$$

où $c_{l, m} \in \mathbb{C}$.

Proposition 5.2. - Supposons $\Pi$ normalisé au degré $\left|l_{1}+m_{1} R\right|-1$ (au sens précisé au début du paragraphe), et supposons $\Pi \in \mathcal{F}$. Soit $Z_{l_{1} m_{1}}$ un champ de vecteurs homogène en $x_{1}^{l_{1}}\left(x^{R}\right)^{m_{1}}$ tel qu'on a (5.1), c'est-à-dire

$$
\left[\Pi^{2}, Z_{l_{1} m_{1}}\right]=c_{l_{1} m_{1}}\left(x^{R}\right)^{m_{1}}\left(x_{1}\right)^{l_{1}} \Pi^{R} .
$$

Alors

(i) $\exp \left(Z_{l_{1} m_{1}}\right)^{*}(\Pi)$ est indépendant du champ $Z_{l_{1}, m_{1}}$ choisi

(ii) $\exp \left(Z_{l_{1} m_{1}}\right)^{*}(\Pi) \in \mathcal{F}$

(iii) $Z_{l_{0} m_{0}}$ s'écrit comme la somme d'un unique champ de vecteurs parallèle à $C$ et d'un multiple quelconque du champ hamiltonien $X_{\Pi^{2}}\left[\left(x^{R}\right)^{m_{0}}\left(x_{1}\right)^{l_{0}}\right]$. Soit $p \in \mathbb{C}$ tel que la composante parallèle à $C$ de $Z_{l_{1} m_{1}}$ s'écrit $p\left(x_{1}\right)^{l_{1}} x^{m_{1} R} C$. Alors

$$
\left[Z_{l_{1} m_{1}}, x^{R} \Pi^{R}\right]=c^{\prime}\left(x_{1}\right)^{l_{1}} x^{\left(m_{1}+1\right) R} \Pi^{R},
$$

avec $c^{\prime}=p\left[m_{1}(C . R)-l_{1} \gamma_{1}\right]$, et $C:=\gamma_{1} Y_{1}+\cdots+\gamma_{n} Y_{n}, C . R=: \gamma_{1} R_{1}+$ $\cdots+\gamma_{n} R_{n}, R:=\left(R_{1}, \ldots, R_{n}\right)$ et $p:=\frac{\gamma_{l_{1}, m_{1}}}{\sigma_{1} L}$ avec $\sigma_{1}$ tel que $S=\sigma_{1} Y_{1}+$ $\cdots+\sigma_{n} Y_{n}$.

Soient $\widehat{\Psi}_{1}:=\cdots \circ \exp \left(Z_{d-1}^{1}\right) \circ \cdots \circ \exp \left(Z_{l}^{1}\right)$ et $\widehat{\Psi}_{2}:=\cdots \circ \exp \left(Z_{d-1}^{2}\right) \circ \cdots \circ$ $\exp \left(Z_{l}^{2}\right)$ deux transformations formelles normalisant $\Pi$ quelconques. Rappelons qu'on note $Z_{d}^{1}$ (resp. $Z_{d}^{2}$ ) des champs de vecteurs s'écrivant sous la forme $\sum_{|I|=d} x^{I} Z^{I}$, où $Z^{I}$ linéaire diagonal. Posons

$$
\Psi_{d}^{1}:=\exp \left(Z_{d}^{1} \circ \cdots \circ \exp \left(Z_{0}^{1}\right), \quad \Psi_{d}^{2}:=\exp \left(Z_{d}^{2}\right) \circ \cdots \circ \exp \left(Z_{0}^{2}\right) .\right.
$$

On a le corollaire suivant de la proposition 5.2. 
Corollaire 5.1. - On a pour tout entier d l'égalité

$$
\left(\Psi_{d}^{1}\right)^{*}(\Pi)=\left(\Psi_{d}^{2}\right)^{*}(\Pi) .
$$

Preuve $d u$ corollaire 5.1. - La relation (5.3) est vraie pour $d=0$ par hypothèse sur $\Pi$ et on a également $\Pi \in \mathcal{F}$ pour $d=0$. On considère la propriété $H(d):\left(\Psi_{d}^{1}\right)^{*}(\Pi)=\left(\Psi_{d}^{2}\right)^{*}(\Pi) \in \mathcal{F}$. Montrons que $H(d)$ implique $H(d+1)$.

Notons $Z_{d}^{1 *}$ (resp. $Z_{d}^{2 *}$ ) la somme des termes non-résonnants de $Z_{d}^{1}$ (resp. de $Z_{d}^{2}$ ). Par le point (i) de la proposition 5.2, on obtient

$$
\left(\exp \left(Z_{d+1}^{1 *}\right) \circ \Psi_{d}^{1}\right)^{*}(\Pi)=\left(\exp \left(Z_{d+1}^{2 *}\right) \circ \Psi_{d}^{2}\right)^{*}(\Pi),
$$

et par le point (ii) de la proposition 5.2, $\left(\Psi_{d+1}^{1}\right)^{*}(\Pi)=\left(\Psi_{d+1}^{1}\right)^{*}(\Pi) \in \mathcal{F}$. La preuve des relations (5.5) et (5.6) achève la preuve du corollaire 5.1.

$$
\begin{aligned}
& \left(\exp \left(Z_{d+1}^{1 *}\right) \circ \Psi_{d}^{1}\right)^{*}(\Pi)=\left(\exp \left(Z_{d+1}^{1}\right) \circ \Psi_{d}^{2}\right)^{*}(\Pi), \\
& \left(\exp \left(Z_{d+1}^{2 *}\right) \circ \Psi_{d}^{1}\right)^{*}(\Pi)=\left(\exp \left(Z_{d+1}^{2}\right) \circ \Psi_{d}^{2}\right)^{*}(\Pi) .
\end{aligned}
$$

On rappelle $(2.5)$ : si $Z$ est un champ de vecteurs formel et $B$ un champ de bivecteurs formel,

$$
\exp (Z)^{*}(B)=\sum_{i=0}^{\infty} \frac{a d_{Z}^{i}(\Pi)}{i !}
$$

où $a d_{Z}$ désigne l'application $[Z,],.[.,$.$] étant le crochet de Schouten-Nijenhuis.$ Comme par hypothèse de récurrence $H(d),\left(\Psi_{d}^{1}\right)^{*}(\Pi)=\left(\Psi_{d}^{1}\right)^{*}(\Pi) \in \mathcal{F}$, le lemme 4.3 implique que s'il existe $j$ avec $j R=d=1$ alors les termes résonnants (éventuels) de $Z_{d+1}^{1}$ et de $Z_{d+1}^{2}$ sont parallèles au champ hamiltonien $X_{\Pi^{R}}\left[\left(x^{R}\right)^{j}\right]$. Si on montre que pour tout entiers $j, k$ et $l$, on a

$$
\left[X_{\Pi^{R}}\left[\left(x^{R}\right)^{j}\right], x_{1}^{l}\left(x^{R}\right)^{k} \Pi^{R}\right]=0,
$$

les relations (5.5) et (5.6) suivent de (5.7), et la preuve du corollaire est achevée. Comme $\Pi^{R}=S_{1} \wedge C$ et que $\mathcal{L}_{S}\left(x^{R}\right)=0$, on a $X_{\Pi^{R}}\left[\left(x^{R}\right)^{j}\right]=-\mathcal{L}_{C}\left[\left(x^{R}\right)^{j}\right] S$. Par (2.9) on en déduit

$$
\begin{aligned}
{\left[X_{\Pi^{R}}\left[\left(x^{R}\right)^{j}\right], x_{1}^{l}\left(x^{R}\right)^{k} \Pi^{R}\right] } & =\left[-\mathcal{L}_{C}\left[\left(x^{R}\right)^{j}\right] S, x_{1}^{l}\left(x^{R}\right)^{k} \Pi^{R}\right] \\
& =\left[-j R . C\left(x^{R}\right)^{j} S, x_{1}^{l}\left(x^{R}\right)^{k} \Pi^{R}\right] \\
& =-j R . C\left(x_{1}^{l}\left(x^{R}\right)^{k}\right) X_{\Pi^{R}}\left[\left(x^{R}\right)^{j}\right] \wedge S \\
& =0 .
\end{aligned}
$$

Preuve de la proposition 5.2. - Par (2.9), $Z_{l_{1} m_{1}}$ est définie à addition d'un multiple du champ hamiltonien de $X_{\Pi^{2}}\left(x_{1}^{l_{1}} x^{m_{1} R}\right)$ près. Pour montrer le point 
(i) de la proposition 5.2 il suffit d'après $(5.7)$ de montrer $\left[X_{\Pi^{2}}\left(\left(x_{1}\right)^{l_{1}} x^{m_{1} R}\right), \Pi\right]=$ 0 . Le monôme $\left(x_{1}\right)^{l_{1}} x^{m_{1} R}$ est non-résonnant, et par $\mathcal{L}_{S_{1}}\left(x^{R}\right)=0$

$$
\begin{aligned}
X_{\Pi^{2}}\left(\left(x_{1}\right)^{l_{1}} x^{m_{1} R}\right) & =\mathcal{L}_{S}\left(\left(x_{1}\right)^{l_{1}} x^{m_{1} R}\right) S_{1} \\
& =l_{1} \sigma_{1}\left(x_{1}\right)^{l_{1}} x^{m_{1} R} S_{1},
\end{aligned}
$$

où on note $S=\sigma_{1} Y_{1}+\cdots+\sigma_{n} Y_{n}$. Rappelons $\mathcal{L}_{S_{1}}\left(x^{R}\right)=0$. D'après (2.9), (5.8), il suit la relation

$$
\left[\left(x_{1}\right)^{l_{1}} x^{m_{1} R} S_{1}, x^{R} \Pi^{R}\right]=x^{R} X_{\Pi^{R}}\left(\left(x_{1}\right)^{l_{1}} x^{m_{1} R}\right) \wedge S_{1}=0,
$$

comme $\Pi^{R}=S_{1} \wedge C$ et $\mathcal{L}_{S_{1}}\left(\left(x_{1}\right)^{l_{1}} x^{m_{1} R}\right)=0$. Le même raisonnement montre qu'on a également pour tout entiers $l, m$ la relation $\left[\left(x_{1}\right)^{l_{1}} x^{m_{1} R} S_{1}\right.$, $\left.\left(x_{1}\right)^{l} x^{m R} \Pi^{R}\right]=0$. D'où le point (i) de la proposition 5.2.

Montrons qu'on peut choisir $Z_{l_{1} m_{1}}$ de la forme $Z_{l_{1} m_{1}}=p\left(x_{1}\right)^{l_{1}} x^{m_{1} R} C$, avec $p$ défini dans l'énoncé. Ensuite montrons

- $\left[Z_{l_{1} m_{1}}, x^{R} \Pi^{R}\right]=c^{\prime}\left(x_{1}\right)^{l_{1}} x^{\left(m_{1}+1\right) R} \Pi^{R}$, où $c^{\prime}=p\left[m_{1}(C . R)-l_{1} \gamma_{1}\right] c$ 'est-àdire le point (iii) de la proposition 5.2.

- pour tout entier $l, m$, on a $\left[Z_{l_{1} m_{1}},\left(x_{1}\right)^{l} x^{m R} \Pi^{R}\right]=\tilde{c}\left(x_{1}\right)^{l_{1}+l} x^{\left(m_{1}+m\right) R} \Pi^{R}$, où $\tilde{c} \in \mathbb{C}$, c'est-à-dire le point (ii) de la proposition 5.2.

Pour montrer que $Z_{l_{1} m_{1}}$ de la forme $p\left(x_{1}\right)^{l_{1}} x^{m_{1} R} C$ convient, montrons $\left[p\left(x_{1}\right)^{l_{1}} x^{m_{1} R} C, \Pi^{2}\right]=c_{l_{1} m_{1}}\left(x_{1}\right)^{l_{1}} x^{m_{1} R} \Pi^{R}$ pour un $p \in \mathbb{C}$. D'après (2.9), $\left[p\left(x_{1}\right)^{l_{1}} x^{m_{1} R} C, \Pi^{2}\right]=X_{\Pi^{2}}\left(p\left(x_{1}\right)^{l_{1}} x^{m_{1} R}\right) \wedge C$ et d'après (5.2), on doit alors avoir

$$
\begin{aligned}
{\left[p\left(x_{1}\right)^{l_{1}} x^{m_{1} R} C, \Pi^{2}\right] } & =p l_{1} \sigma_{1}\left(x_{1}\right)^{l_{1}} x^{m_{1} R} S_{1} \wedge C \\
& =c_{l_{1} m_{1}}\left(x_{1}\right)^{l_{1}} x^{m_{1} R} \Pi^{R} .
\end{aligned}
$$

Comme $\Pi^{R}=S_{1} \wedge C$, ceci conduit à $p=\frac{c_{l_{1} m_{1}}}{\sigma_{1} l_{1}}$. On peut donc bien choisir $Z_{l_{1} m_{1}}$ de la forme $Z_{l_{1} m_{1}}=\frac{c_{l_{1} m_{1}}}{\sigma_{1} l_{1}}\left(x_{1}\right)^{l_{1}} x^{m_{1} R} C$.

Montrons qu'on a $\left[Z_{l_{1}, m_{1}}, x^{R} \Pi^{R}\right]=c^{\prime}\left(x_{1}\right)^{l_{1}} x^{\left(m_{1}+1\right) R} \Pi^{R}$, où $c^{\prime}=p\left[m_{1}(C . R)-\right.$ $\left.l_{1} \gamma_{1}\right]$. D'après (2.9),

$$
\begin{aligned}
{\left[Z_{l_{1}, m_{1}}, x^{R} \Pi^{R}\right] } & =\left[p\left(x_{1}\right)^{l_{1}} x^{m_{1} R} C, x^{R} \Pi^{R}\right] \\
& =p C \wedge x^{R} X_{\Pi^{R}}\left[\left(x_{1}\right)^{l_{1}} x^{m_{1} R}\right]+p\left(x_{1}\right)^{l_{1}} x^{m_{1} R} \mathcal{L}_{C}\left(x^{R}\right) \Pi^{R}
\end{aligned}
$$

Avec $X_{\Pi^{R}}\left(\left(x_{1}\right)^{l_{1}} x^{m_{1} R}\right)=\mathcal{L}_{S_{1}}\left[\left(x_{1}\right)^{l_{1}} x^{m_{1} R}\right] C-\mathcal{L}_{C}\left[\left(x_{1}\right)^{l_{1}} x^{m_{1} R}\right] S_{1}=$ $-\mathcal{L}_{C}\left[\left(x_{1}\right)^{l_{1}} x^{m_{1} R}\right] S_{1}$, il suit

$$
\begin{aligned}
{\left[Z_{l_{1} m_{1}}, x^{R} \Pi^{R}\right] } & =p x^{R} \mathcal{L}_{C}\left[\left(x_{1}\right)^{l_{1}} x^{m_{1} R}\right] C \wedge S_{1}+p\left(x_{1}\right)^{l_{1}} x^{m_{1} R} \mathcal{L}_{C}\left(x^{R}\right) \Pi^{R} \\
& =-p x^{R} \mathcal{L}_{C}\left[\left(x_{1}\right)^{l_{1}} x^{m_{1} R}\right] \Pi^{R}+p\left(x_{1}\right)^{l_{1}} x^{m_{1} R} \mathcal{L}_{C}\left(x^{R}\right) \Pi^{R} \\
& =p\left[m_{1}(C . R)-\left(l_{1} \gamma_{1}\right)\right]\left(x_{1}\right)^{l_{1}} x^{\left(m_{1}+1\right) R} \Pi^{R}
\end{aligned}
$$

où on note $C:=\gamma_{1} Y_{1}+\cdots+\gamma_{n} Y_{n}, C \cdot R=: \gamma_{1} R_{1}+\cdots+\gamma_{n} R_{n}$ avec $R:=$ $\left(R_{1}, \ldots, R_{n}\right)$. D'où le point (iii) de la proposition 5.2. 
Montrons que pour tout entier $l, m, \quad\left[Z_{l_{1} m_{1}},\left(x_{1}\right)^{l} x^{m R} \Pi^{R}\right]=$ $\tilde{c}\left(x_{1}\right)^{l_{1}+l} x^{(K+m) R} \Pi^{R}$, où $\tilde{c} \in \mathbb{C}$. D'après (2.9),

$$
\begin{aligned}
{\left[Z_{l_{1} m_{1}},\left(x_{1}\right)^{l} x^{m R} \Pi^{R}\right]=} & {\left[p\left(x_{1}\right)^{l_{1}} x^{m_{1} R} C,\left(x_{1}\right)^{l} x^{m R} \Pi^{R}\right] } \\
= & p C \wedge\left(x_{1}\right)^{l} x^{m R} X_{\Pi^{R}}\left(\left(x_{1}\right)^{l_{1}} x^{m_{1} R}\right) \\
& +p\left(x_{1}\right)^{l_{1}} x^{m_{1} R} \mathcal{L}_{C}\left(\left(x_{1}\right)^{l} x^{m R}\right) \Pi^{R}
\end{aligned}
$$

Or,

$$
\begin{aligned}
X_{\Pi^{R}}\left(\left(x_{1}\right)^{l_{1}} x^{m_{1} R}\right) & =\mathcal{L}_{S_{1}}\left(\left(x_{1}\right)^{l_{1}} x^{m_{1} R}\right) C-\mathcal{L}_{C}\left(\left(x_{1}\right)^{l_{1}} x^{m_{1} R}\right) S_{1} \\
& =-\mathcal{L}_{C}\left(\left(x_{1}\right)^{l_{1}} x^{m_{1} R}\right) S_{1}
\end{aligned}
$$

avec

$$
\begin{aligned}
{\left[Z_{l_{1} m_{1}},\left(x_{1}\right)^{l} x^{m R} \Pi^{R}\right] } & =-p C \wedge\left(x_{1}\right)^{l} x^{m R} \mathcal{L}_{C}\left(\left(x_{1}\right)^{l_{1}} x^{m_{1} R}\right) S_{1} \\
& +p\left(x_{1}\right)^{l_{1}} x^{m_{1} R} \mathcal{L}_{C}\left(\left(x_{1}\right)^{l} x^{m R}\right) \Pi^{R} \\
& =2 p\left(l_{1} \gamma_{1}+m R . C\right)\left(x_{1}\right)^{\left(l_{1}+l\right)} x^{\left(m_{1}+m\right) R} \Pi^{R} .
\end{aligned}
$$

D'où le point (ii) de la proposition 5.2.

Preuve de la proposition 5.1. - Soit $\hat{Z}$ un champ de vecteurs formel quelconque tel que $\exp (\hat{Z})^{*}(\Pi)=\Pi^{2}+x^{R} \Pi^{R}$, où $\Pi:=\Pi^{2}+x^{R} \Pi^{R}+\left(x_{1}\right)^{l_{0}} \Pi^{R}$. Considérons les termes $Z^{\left(m, l_{0}\right)}$ en $\left(x_{1}\right)^{l_{0}} x^{m R}, m \in \mathbb{N}$ de $\hat{Z}$. D'après $(5.7), Z^{\left(0, l_{0}\right)}$ doit satisfaire $\left[Z^{\left(0, l_{0}\right)}, \Pi^{2}\right]=\left(x_{1}\right)^{l_{0}} \Pi^{R}$. Après $Z^{\left(1, l_{0}\right)}$ doit vérifier $\left[Z^{\left(1, l_{0}\right)}, \Pi^{2}\right]=$ $\left[Z^{\left(0, l_{0}\right)}, x^{R} \Pi^{R}\right]$, et, en itérant, on obtient que $Z^{\left(m, l_{0}\right)}$ doit vérifier l'équation

$$
\left[Z^{\left(m, l_{0}\right)}, \Pi^{2}\right]=\left[Z^{(m-1), l_{0}}, x^{R} \Pi^{R}\right] .
$$

D'après la proposition 5.2 et le corollaire 5.1 , on sait que

- pour tout $m,\left[Z^{(m-1), l_{0}}, x^{R} \Pi^{R}\right]$ est de la forme $c^{\left(m, l_{0}\right)}\left(x_{1}\right)^{l_{0}} x^{m R} \Pi^{R}$,

- la composante parallèle à $C$ de $Z^{\left(m, l_{0}\right)}$ est la même pour toute transformation formelle normalisant $\Pi$, et elle vaut $\frac{c^{\left(m, l_{0}\right)}}{\sigma_{1} l_{0}}\left(x_{1}\right)^{l_{0}} x^{m R} C$,

- on a alors $\left[Z^{\left(m, l_{0}\right)}, x^{R} \Pi^{R}\right]=\frac{c^{\left(m, l_{0}\right)}}{\sigma_{1} l_{0}}\left[m(C . R)-\left(l_{0} \gamma_{1}\right)\right]\left(x_{1}\right)^{l_{0}} x^{(m+1) R} \Pi^{R}$.

On en déduit la relation de récurrence

$$
c^{\left(m+1, l_{0}\right)}=c^{\left(m, l_{0}\right)}\left(\frac{m(C \cdot R)-\left(l_{0} \gamma_{1}\right)}{\sigma_{1} l_{0}}\right) .
$$

Il existe un entier $m_{0}$, tel que pour $m$ assez grand

$$
\frac{m(C . R)}{\sigma_{1} l_{0}}>\left|\frac{m(C . R)}{\sigma_{1} l_{0}}-\gamma_{1}\right|>\frac{\left(m-m_{0}\right)(C . R)}{\sigma_{1} l_{0}} .
$$

On en déduit pour $m$ assez grand

$$
m !\left(\frac{C . R}{\sigma_{1} l_{0}}\right)>c^{\left(m, l_{0}\right)}>\left(m-m_{0}\right) !\left(\frac{C . R}{\sigma_{1} l_{0}}\right)^{m} .
$$


Or, comme la composante parallèle à $C$ de $Z^{\left(m, l_{0}\right)}$ est bien déterminée et vaut $\frac{c^{\left(m, l_{0}\right)}}{s_{1} l_{0}}\left(x_{1}\right)^{l_{0}} x^{m R} C,(5.10)$ montre bien la divergence en $\left(x_{1}\right)^{l_{0}} x^{m R}$ de tout champ formel $\hat{Z}$ tel que $\exp \hat{Z}$ normalise $\Pi$. De plus, si on choisit chaque $Z^{\left(m, l_{0}\right)}$ parallèle à $C$, la série des termes en $\left(x_{1}\right)^{l_{0}} x^{m R}$ de $\hat{Z}$ est 1 -Gevrey. On peut de même montrer, pour tout entier $k$ fixé, que la somme des termes en $\left(x_{1}\right)^{k l_{0}} x^{m R}$ de $\hat{Z}$ est divergente et s'ils sont choisis parallèles à $C$ ces séries sont 1-Gevrey.

\section{Prénormalisation à la Dulac}

Proposition 6.1 (prénormalisation à la Dulac). - Soit une structure de Poisson $\Pi$ telle que dans la proposition 4.1. Supposons toutes les composantes de $R$ strictement positives (hypothèse $\left(P_{5}\right)$, théorème 3.1) et que la partie quadratique $\Pi^{2}$ de $\Pi$ satisfait condition (D) (cf. section 3). Alors il existe une transformation $\Phi:\left(\mathbb{C}^{n}, 0\right) \rightarrow\left(\mathbb{C}^{n}, 0\right)$ holomorphe telle que

$$
\Phi^{*}(\Pi)=\Pi^{2}+x^{R} \Pi^{R}+x^{R} \mathfrak{R}, \quad \mathfrak{R}=\sum_{I \in \mathbb{N}^{n}} x^{I} \Pi^{I},
$$

où on désigne par $\Pi^{I}$ des champs de bivecteurs quadratiques diagonaux.

Remarque. - Les multiindices $I$ apparaissant effectivement dans la somme n'ont pas de composante strictement négative.

Notations. On désigne par $\mathfrak{J}$ (resp. $\tilde{\mathfrak{J}}$ ) l'ensemble des champs de bivecteurs $B$ formels (resp. champs de vecteurs $V$ ) sur $\mathbb{C}^{n}$ s'écrivant $B=x^{R} \sum_{J} x^{J} \Pi^{R+J}$ (resp. $V=x^{R} \sum_{J} x^{J} V^{R+J}$ ), où on note $\Pi^{R+J}$ des champs de bivecteurs quadratiques diagonaux, (resp. $V^{R+J}$ des champ de vecteurs linéaires diagonaux) et où aucun terme $x^{J} \Pi^{R+J}$ (resp. $\sum_{J} x^{J} V^{R+J}$ ) apparaissant effectivement dans la somme est analytique en $0 \in \mathbb{C}^{n}$. On remarque que les multiindices $J$ peuvent néanmoins avoir des composantes égales à -1 (deux dans le cas des champs de bivecteurs, une dans le cas des champs de vecteurs).

On désigne par $\mathfrak{J}_{1}$ l'ensemble des champs de bivecteurs $B$ formels (resp. champs de vecteurs $V$ ) sur $\mathbb{C}^{n}$ s'écrivant $B=x^{R} \sum_{J \in \mathbb{N}^{n}} x^{J} \Pi^{R+J}$, (resp. $V=$ $x^{R} \sum_{J \in \mathbb{N}^{n}} x^{J} V^{R+J}$ ) où on note $\Pi^{R+J}$ des champs de bivecteurs quadratique diagonaux (resp $V^{R+J}$ des champs de vecteurs linéaires diagonaux). On remarque que les multiindices $J$ n'ont pas de composante égale à -1 .

On désigne par $\mathcal{J}$ l'idéal des fonctions formelles $\mathbb{C}^{n} \rightarrow \mathbb{C}$ divisibles par $x^{R}$, c'est-à-dire $f \in \mathcal{J} \Rightarrow f=x^{R} \sum_{I \in \mathbb{N}^{n}} a^{I} x^{I}, a^{I} \in \mathbb{C}$.

Définition 6.1. - On dit qu'une structure de Poisson $\Pi$, telle qu'en la proposition 6.1, est 0-prénormalisée si

$$
\Pi=\Pi^{2}+x^{R} \Pi^{R}+x^{R} \mathcal{R} \text {, où } x^{R} \mathcal{R} \in \mathfrak{J} .
$$


Plan de la preuve de la proposition 6.1. On montre d'abord l'existence d'un changement de coordonnées $\Phi_{1} 0$-prénormalisant. Dans un second temps on montre comment passer de $\Pi$ sous forme 0-prénormalisé à la forme prénormalisé au sens de la proposition 6.1.

Lemme 6.1. - Soit $\Pi$ tel que dans la proposition 6.1. Alors il existe une transformation formelle $\hat{\Phi}_{1}:\left(\mathbb{C}^{n}, 0\right) \rightarrow\left(\mathbb{C}^{n}, 0\right)$ conjuguant $\Pi$ à une structure de Poisson 0-prénormalisé.

Démonstration. - Supposons $\Pi$ 0-prénormalisé au degré $d$, i.e.

$$
\Pi=\Pi^{2}+x^{R} \Pi^{R}+x^{R} \sum_{|R|<|I| \leq d-|R|} x^{I} \Pi^{R+I}+R^{d},
$$

où les termes de $R^{d}$ sont de degrés strictement supérieurs à $d+2$.

Soit alors $x^{I_{0}} \Pi^{I_{0}}$ un des terme de plus petit degré de $\mathfrak{R}$ n'appartenant pas à $\mathfrak{J}$, i.e. $x^{I_{0}-R} \Pi^{I_{0}}$ n'est pas analytique en $0 \in \mathbb{C}^{n}$. Alors $x^{I_{0}} \notin \mathcal{J}$ et on déduit des lemmes 2.1 et 6.2 que l'identité de Jacobi $[\Pi, \Pi]=0$ implique la relation

$$
\left[\Pi^{2}, x^{I_{0}} \Pi^{I_{0}}\right]=0 .
$$

Comme l'hypothèse $(\mathrm{H})$ est satisfaite, on obtient comme dans la démonstration de la proposition 2.1 (cf. [5], section 3) l'existence d'un champ de vecteurs analytique de la forme $x^{I_{0}} Z$, avec $Z$ linéaire diagonal tel que

$$
\left[\Pi^{2}, x^{I} Z\right]=x^{I} \Pi^{I} \text {. }
$$

Par (2.5) on en déduit que $\Pi$ est 0 -prénormalisable au degré $l+1$ par le moyen d'une transformation $\exp (\hat{T})$, où $T$ est un champ de vecteurs homogène de degré $\left|I_{0}\right|+1$, et en itérant, intégralement 0-prénormalisable.

Lemme 6.2. - Soient $x^{R} x^{I} \Pi^{I}, x^{R} x^{J} \Pi^{J} \in \mathfrak{J}$. Alors $\left[x^{R} x^{I} \Pi^{I}, x^{R} x^{J} \Pi^{J}\right]$ s'écrit comme une somme de termes de la forme $x^{K} Y_{i} \wedge Y_{j} \wedge Y_{k}$, où $x^{K} \in \mathcal{J}$.

Preuve du lemme 6.2. - D'après (2.2) et (2.4), on a la relation

$$
\begin{aligned}
{\left[x^{R} x^{I} \Pi^{I}, x^{R} x^{J} \Pi^{J}\right] } & =x^{2 R}\left[x^{I} \Pi^{I}, x^{J} \Pi^{J}\right]+x^{R} x^{I} X_{\Pi^{I}}\left(x^{R}\right) \wedge x^{J} \Pi^{J} \\
& +x^{R} x^{J} X_{\Pi^{J}}\left(x^{R}\right) \wedge x^{I} \Pi^{I}
\end{aligned}
$$

Le crochet de Schouten $\left[x^{I} \Pi^{I}, x^{J} \Pi^{J}\right]$ est analytique en $0 \in \mathbb{C}^{n}$ et s'écrit comme somme de termes de la forme $a_{i j k} x^{K} Y_{i} \wedge Y_{j} \wedge Y_{k}$ tels que la $i, j$, ou $k$-ème composante de $K$ peut prendre la valeur -1 . Par suite $x^{2 R}\left[x^{I} \Pi^{I}, x^{J} \Pi^{J}\right]$ s'écrit comme somme de termes de la forme $a_{i j k} x^{R} x^{K^{\prime}} Y_{i} \wedge Y_{j} \wedge Y_{k}$ tels que $K^{\prime}=K+R$ n'a pas de composantes strictement négative puisque les composantes de $R$ sont par hypothèse strictement positives.

Considérons les termes $x^{R} x^{I} X_{\Pi^{I}}\left(x^{R}\right) \wedge x^{J} \Pi^{J}$ et $x^{R} x^{J} X_{\Pi^{J}}\left(x^{R}\right) \wedge x^{I} \Pi^{I}$ de (6.4). Comme $\Pi^{I}$ et $\Pi^{J}$ sont des champs de bivecteurs quadratiques diagonaux, 
les champs hamiltoniens $X_{\Pi^{I}}\left(x^{R}\right)$ et $X_{\Pi^{J}}\left(x^{R}\right)$ s'écrivent comme des produits de $x^{R}$ par un champ de vecteurs linéaire diagonal.

Il suit que dans les cas où $I$ et $J$ n'ont pas de composante égale à -1 , ou bien s'il n'existe pas d'indice $1 \leq i \leq n$ tel que les $i$-èmes coordonnées de $I$ et $J$ prennent simultanément la valeur $-1, x^{R} x^{I} X_{\Pi^{I}}\left(x^{R}\right) \wedge x^{J} \Pi^{J}$ et $x^{R} x^{J} X_{\Pi^{J}}\left(x^{R}\right) \wedge$ $x^{I} \Pi^{I}$ s'écrivent comme somme de termes de la forme $a_{i j k} x^{R} x^{K^{\prime}} Y_{i} \wedge Y_{j} \wedge Y_{k}$ tels que $K^{\prime}=K+R$ n'a pas de composante égale à -1 , car par hypothèse les composantes de $R$ sont strictement positives. Donc $x^{R} x^{K^{\prime}} \in \mathcal{J}$.

Pour montrer le lemme 6.2 il reste donc à traiter le cas où il existe $1 \leq i_{0} \leq n$ tel que les $i_{0}$-èmes composantes de $I$ et de $J$ prennent simultanément la valeur -1 . Comme $x^{I} \Pi^{I}$ et $x^{J} \Pi^{J}$ sont par hypothèse analytiques en $0 \in \mathbb{C}^{n}$ il suit

$$
\Pi^{I}=B_{i_{0}}^{I} \wedge Y_{i_{0}}, \quad \Pi^{J}=B_{i_{0}}^{J} \wedge Y_{i_{0}} .
$$

On a alors, d'après (2.6)

$$
\begin{aligned}
x^{R} x^{I} X_{\Pi^{I}}\left(x^{R}\right) \wedge x^{J} \Pi^{J} & =x^{R} x^{I}\left(\mathcal{L}_{B_{i_{0}}^{I}}\left(x^{R}\right) Y_{i_{0}}\right) \wedge x^{J} B_{i_{0}}^{J} \wedge Y_{i_{0}} \\
& -x^{R} x^{I}\left(\mathcal{L}_{Y_{i_{0}}}\left(x^{R}\right) B_{i_{0}}^{I}\right) \wedge x^{J} B_{i_{0}}^{J} \wedge Y_{i_{0}} \\
& =-x^{R} x^{I}\left(\mathcal{L}_{Y_{i_{0}}}\left(x^{R}\right) B_{i_{0}}^{I}\right) \wedge x^{J} B_{i_{0}}^{J} \wedge Y_{i_{0}}
\end{aligned}
$$

De même, $x^{R} x^{J} X_{\Pi^{J}}\left(x^{R}\right) \wedge x^{I} \Pi^{I}=-x^{R} x^{J}\left(\mathcal{L}_{Y_{i_{0}}}\left(x^{R}\right) B_{i_{0}}^{J}\right) \wedge x^{J} B_{i_{0}}^{I} \wedge Y_{i_{0}}$. Il suit que la somme de $x^{R} x^{I} X_{\Pi^{I}}\left(x^{R}\right) \wedge x^{J} \Pi^{J}$ et de $x^{R} x^{J} X_{\Pi^{J}}\left(x^{R}\right) \wedge x^{I} \Pi^{I}$ est nulle s'il existe $1 \leq i_{0} \leq n$ tel que les $i_{0}$-èmes composantes de $I$ et de $J$ prennent la valeur -1 . D'où le lemme 6.2.

Equation cohomologique modulo $\mathfrak{J}$, petits diviseurs associés. On donne des résultats préliminaires utilisées das la preuve de la convergence des transformations 0-prénormalisantes.

Notations. Rappelons $\Pi^{2}=\frac{1}{2} \sum_{i \neq j} a_{i j} Y_{i} \wedge Y_{j}$, avec $a_{i j}=-a_{j i}$ et posons $A_{i}:=\sum_{j=1}^{n} a_{i j} Y_{j}$. On a $\mathbb{S}:=\operatorname{Vect}\left\langle S_{1}, S_{2}\right\rangle=\operatorname{Vect}\left\langle A_{1}, \ldots, A_{n}\right\rangle$ (cf. hypothèse $(P 2)$, théorème 3.1$)$.

Un multiindice $I \in(\mathbb{N} \cup\{-1\})^{n}$ définit une application linéaire $\beta^{I}: \mathbb{S} \rightarrow \mathbb{C}$ telle que $\mathcal{L}_{A_{i}}\left(x^{I}\right)=\beta^{I}\left(A_{i}\right) x^{I}$. Notons $\beta_{i}^{I}$ le nombre complexe $\beta^{I}\left(A_{i}\right)$. Soit $I \in(\mathbb{N} \cup\{-1\})^{n}$ un multiindice tel que $x^{I} \notin \mathcal{J}$. Posons

$$
E_{I}:=\left\{1 \leq i \leq n \mid y_{i} y^{I} \notin \mathcal{J}\right\}
$$

Fixons un multiindice $I$ (avec au plus une seule composante égale à -1 ). Soit $\Pi^{I}:=\sum_{i \neq j} a_{i j}^{I} Y_{i} \wedge Y_{j}$ avec $a_{i j}^{I}=-a_{j i}^{I}$ un champ de bivecteurs quadratique diagonal. Supposons $a_{i j}^{I}$ non nul uniquement dans le cas où $i$ et $j$ appartiennent tous les deux à $E_{I}$. On dit qu'un champ de vecteurs $x^{I} Z$ avec $Z$ linéaire diagonal 
vérifie propriété $(\mathrm{P})$ s'il existe un champ de bivecteurs quadratique diagonal $\Pi^{\prime}(Z) \in \mathfrak{J}$ tel que

$$
\left[\Pi^{2}, x^{I} Z\right]=x^{I} \Pi^{I}+x^{I} \Pi^{\prime}(Z) .
$$

Remarque. - Le champ de bivecteurs $\Pi^{\prime}(Z)$ appartient à $\mathfrak{J}$ signifie qu'il s'écrit $\Pi^{\prime}(Z)=\sum_{\substack{k \notin E_{I} \\ i \neq k}} c_{i, k} Y_{i} \wedge Y_{k}$. La propriété $(P)$ se rencontre lors qu'on effectue une 0-prénormalisation du degré $d$ au degré $d+1:$ au lieu de résoudre une équation cohomologique classique on résout une équation modulo $\mathfrak{J}$.

Si $Z$ vérifie $(C)$ on a d'après (2.9), $x^{I} \Pi^{I}+x^{I} \Pi^{\prime}=Z \wedge X_{\Pi^{2}}\left(x^{I}\right)$. Or, on vérifie $X_{\Pi^{2}}\left(x^{I}\right)=x^{I} \sum_{1 \leq i \leq n} \beta_{i}^{I} Y_{i}$. D'où

$$
x^{I} \Pi^{I}+x^{I} \Pi^{\prime}=Z \wedge x^{I} \sum_{1 \leq i \leq n} \beta_{i}^{I} Y_{i} .
$$

On déduit de (6.7) que si $x^{I} Z$ vérifie (P), alors on a les deux propriétés suivantes.

- Pour tout $c \in \mathbb{C}$, le champ de vecteurs $x^{I} Z+c x^{I} \sum_{1 \leq i \leq n} \beta_{i}^{I} Y_{i}$ vérifie encore $(\mathrm{P})$ (dans la mesure où $x^{I} \sum_{1<i<n} \beta_{i}^{I} Y_{i}$ est analytique - ce qui n'est pas le cas en général si $I$ comporte une composante égale à -1$)$;

- Soit $i \notin E_{I}$ et posons $x^{I} Z:=x^{I}\left(z_{1} Y_{1}, \ldots, z_{i} Y_{i}, \ldots, z_{n} Y_{n}\right)$. Alors $x^{I} Z^{\prime}:=$ $x^{I}\left(z_{1} Y_{1}, \ldots, 0, \ldots, z_{n} Y_{n}\right)$ vérifie encore $(P)$.

On est donc amené à distinguer le cas où $I$ ne comporte pas de composante strictement négative, et celui où $I$ comporte une composante égale à -1 .

On considère d'abord le cas où $I$ ne comporte pas de composante égale à -1 . Dans ce cas le champ hamiltonien $X_{\Pi^{2}}\left(x^{I}\right)$ est analytique et quitte à ajouter un multiple approprié de $X_{\Pi^{2}}\left(x^{I}\right)$ on peut choisir $x^{I} Z$ avec la $i_{0}$-ème coordonnée nulle pour $i_{0} \in E_{I}$ tel que $\beta_{i_{0}}^{I} \neq 0$. On peut supposer en plus que les $k$-èmes coordonnées de $Z$, pour $k \notin E_{I}$ soient nulles.

Comme les $k$-èmes coordonnées de $Z$ sont nulles pour $k \notin E_{I}$ ainsi que pour $k=i_{0}$, la propriété $(\mathrm{P})$ et l'équation (6.7) imposent l'égalité suivante pour les termes de $\Pi^{I}$ ayant $Y_{i_{0}}$ en facteur du produit extérieur :

$$
\begin{aligned}
\beta_{i_{0}}^{I} Z \wedge Y_{i_{0}} & =\frac{1}{2} \sum_{j \in E_{I}}\left(a_{i_{0} j}^{I}-a_{j i_{0}}^{I}\right) Y_{j} \\
& =\sum_{j \in E_{I}}^{n} a_{i_{0} j}^{I} Y_{j} .
\end{aligned}
$$

On en déduit 
LEMME 6.3. - Soit $I \in \mathbb{N}^{n}$ (sans composante négative). Supposons qu'il existe $x^{I} Z^{*}$ vérifiant propriété $(P)$. Alors on peut choisir $x^{I} Z:=x^{I} z_{1} Y_{1}+\cdots+z_{n} Y_{n}$ vérifiant $(P)$ tel que pour $k \notin E_{I}$ les $k$-èmes coordonnées de $Z$ soient nulles, et tel que

$$
\max _{1 \leq j \leq n}\left|z_{j}\right| \leq \frac{n}{\left|\beta^{I}\right|} \max _{j_{0} \neq i}\left(\left|a_{i j_{0}}^{I}\right|\right)
$$

où on note $\left|\beta^{I}\right|=\max _{i \in E_{I}}\left|\beta_{i}^{I}\right|$.

On considère maintenant le cas où $I$ admet une composante égale à -1 . Supposons que ce soit la $j_{0}$-ème. Les seuls champs de vecteurs en $x^{I}$ qui sont analytiques en $0 \in \mathbb{C}^{n}$ sont alors les multiples de $x^{I} Y_{j_{0}}$ et on a $j_{0} \notin E_{I}$. Supposons qu'il existe $x^{I} Z:=x^{I} z_{j_{0}} Y_{j_{0}}$ vérifiant $(\mathrm{P})$. D'après $(6.7)$ on a $x^{I}\left(\Pi^{I}+\Pi^{\prime}\right)=$ $z_{j_{0}} Y_{j_{0}} \wedge x^{I} \sum_{i \neq j_{0}} \beta_{i}^{I} Y_{i}$. On en déduit $\left|z_{j_{0}}\right| \leq\left(\max _{\substack{i \neq j_{0} \\ i \in E_{I}}}\left(\left|\beta_{i}^{I}\right|\right)\right)^{-1} \max _{i \neq j_{0}}\left(\left|a_{i, j_{0}}^{I}\right|\right)$. Si $E_{I}=\{1, \ldots, n\}$ il suit du lemme 4.3 de [9] la relation $\left(\max _{\substack{i \neq j_{0} \\ i \in E_{I}}}\left(\left|\beta_{i}^{I}\right|\right)\right)^{-1} \leq$ $\left(\left|\beta^{I}\right|\right)^{-1} n|I|$. Or, s'il existe $1 \leq i_{0} \leq n$ avec $i_{0} \notin E_{I}$, alors il existe $c>0$ ne dépendant pas de $|I|$ tel que $\left(\max _{\substack{i \neq j_{0} \\ i \in E_{I}}}\left(\left|\beta_{i}^{I}\right|\right)\right)^{-1} \leq c$. En effet on a $\mathcal{L}_{A_{i}}\left(x_{i_{0}} x^{I}\right)=0$ pour tout $1 \leq i \leq n$. Il suit $\left(1 / x^{I}\right) \mathcal{L}_{A_{i}}\left(x^{I}\right)=-a_{i i_{0}}$ et on vérifie facilement qu'on aboutit à une contradiction si pour $i \in E_{I}$ on a $a_{i i_{0}}=0$. On en déduit

LEMME 6.4. - Soit $I \in(\mathbb{N} \cup\{-1\})^{n}$ un multiindice avec la $j_{0}$-ème composante soit égale à -1 , les autre étant positives ou nulles. Supposons que $x^{I} Z:=$ $x^{I} \sum_{1 \leq i \leq n} z_{i} Y_{i}$ vérifie la propriété $(P)$. Alors on a $x^{I} Z=x^{I} z_{j_{0}} Y_{j_{0}}$ et il existe $c>0$ tel que

$$
\left|z_{j_{0}}\right| \leq \frac{n|I| c}{\left|\beta^{I}\right|} \max _{j_{0} \neq i}\left(\left|a_{i j_{0}}^{I}\right|\right)
$$

où on note $\left|\beta^{I}\right|=\max _{i \in E_{I}}\left|\beta_{i}^{I}\right|$.

LEMME 6.5. - Sous les hypothèses de la proposition 6.1,

$$
-\sum_{k \geq 0} \frac{\ln \omega_{k}^{\prime}}{2^{k}}<+\infty
$$

avec $\omega_{k}^{\prime}:=\inf \left\{\max _{i \in E_{I}}\left|\beta_{i}^{I}\right| ;|I| \leq 2^{k}, y^{I} \notin \mathcal{J}\right\}$.

Preuve du lemme 6.5. - On peut formuler la condition (D) comme suit : $-\sum_{k \geq 0} \frac{\ln \omega_{k}}{2^{k}}<+\infty$, avec $\omega_{k}:=\inf \left\{\max _{1 \leq i \leq n}\left|\beta_{i}^{I}\right| ; 1 \leq i \leq n,|I| \leq 2^{k}, x^{I} \notin \mathcal{J}\right\}$. On a alors (6.11) pour les raisons suivantes : 
- pour $I$ tels que $E_{I}=\{1, \ldots, n\}$ on a $\max _{1 \leq i \leq n}\left|\beta_{i}^{I}\right|=\max _{i \in E_{I}}\left|\beta_{i}^{I}\right|$;

- pour $I$ tels que $y^{I} \notin \mathcal{J}$, mais tels qu'il existe $1 \leq i_{0} \leq n$ avec $y^{I} \in \mathcal{J}$, on a vu plus haut qu'il existe $c>0$ tel que $\left|\beta^{I}\right|=\max _{i \in E_{I}}\left|\beta_{i}^{I}\right|>c$.

Convergence de transformations 0-prénormalisantes. Ce qui suit est la transposition de [15] aux structures de Poisson à 1-jet nul et à partie quadratique non nulle.

Soit $\Pi$ la structure de Poisson de la proposition 6.1. Notons $\{., .\}_{\Pi}$ le crochet associé, que nous supposons donné par le système

$$
\left\{x_{i}, x_{j}\right\}_{\Pi}=a_{i j} x_{i} x_{j}+f_{i j}(x) x_{i} x_{j}, \quad 1 \leq i<j \leq n,
$$

où $f_{i j}(x) x_{i} x_{j}$ est analytique en $0 \in \mathbb{C}^{n}$. Précisons que dans notre notation, les $f_{i j}$ peuvent éventuellement avoir des termes méromorphes, mais pas $x_{i} x_{j} f_{i j}$. On montre l'existence d'une 0-prénormalisation sous la forme du lemme

Lemme 6.6. - Il existe un système de coordonnées locales analytique $x=$ $y+\xi(y)$ dans lequel le système (6.12) s'écrit

$$
\left\{y_{i}, y_{j}\right\}_{\Pi}=a_{i j} y_{i} y_{j}+x^{R} g_{i j}(y) y_{i} y_{j}, \quad 1 \leq i<j \leq n,
$$

où $g_{i j} y_{i} y_{j}$ est analytiques en $0 \in \mathbb{C}^{n}$.

Preuve du lemme 6.6. - Effectuons dans le système (6.12) un changement de variable formel tangent à l'identité au voisinage de $0, x=y+\xi(y), \xi(y)=$ $\left(y_{i} \xi_{j}(y)\right)_{i=1, \ldots, n}$, c'est-à-dire

$$
x_{j}=y_{j}+y_{j} \xi_{j}(y), \quad \text { où } \xi_{j}=\sum_{|Q|>|R|} \xi_{j, Q} y^{Q},
$$

où la $j$-ème coordonnée de $Q$ peut éventuellement être égale à -1 . On obtient un système de la forme

$$
\left\{y_{i}, y_{j}\right\}_{\Pi}=a_{i j} y_{i} y_{j}+y_{i} y_{j} \psi_{i j}(y), \quad 1 \leq i<j \leq n .
$$

Posons $\psi_{i j}=\sum_{|Q| \geq|R|} \psi_{i j, Q} y^{Q}$. Remarquons que $\psi_{i j, Q} y^{Q} y_{i} y_{j}$ est analytique en $0 \in \mathbb{C}^{n}$.

Relation entre $\xi, f$ et $\psi$. - D'après (6.15) et (6.14), on a pour tout $i<j$

$$
\begin{aligned}
\left\{x_{i}, x_{j}\right\}_{\Pi} & =\left\{y_{i}, y_{j}\right\}_{\Pi}+\left\{y_{i} \xi_{i}, y_{j}\right\}_{\Pi}+\left\{y_{i}, y_{j} \xi_{j}\right\}_{\Pi}+\left\{y_{i} \xi_{i}, y_{j} \xi_{j}\right\}_{\Pi} \\
& =\left\{y_{i}, y_{j}\right\}_{\Pi_{2}}+\left\{y_{i} \xi_{i}, y_{j}\right\}_{\Pi_{2}}+\left\{y_{i}, y_{j} \xi_{j}\right\}_{\Pi_{2}}+\left\{y_{i} \xi_{i}, y_{j} \xi_{j}\right\}_{\Pi_{2}} \\
& +\left\{y_{i}, y_{j}\right\}_{\psi}+\left\{y_{i} \xi_{i}, y_{j}\right\}_{\psi}+\left\{y_{i}, y_{j} \xi_{j}\right\}_{\psi}+\left\{y_{i} \xi_{i}, y_{j} \xi_{j}\right\}_{\psi} .
\end{aligned}
$$

Ici le crochet $\{., .\}_{\Psi}$ est défini par

$$
\{f, g\}_{\Psi}=\sum_{i<j} y_{i} y_{j} \Psi_{i j}(y)\left[\partial_{y_{i}}(f) \partial_{y_{j}}(g)-\partial_{y_{i}}(g) \partial_{y_{j}}(f)\right] .
$$


(Comme on a pas l'identité de Jacobi, cette notation ne désigne pas un crochet de Poisson). Or, d'après (6.12) et (6.14) on a

$$
\begin{aligned}
\left\{x_{i}, x_{j}\right\}_{\Pi} & =a_{i j}\left(y_{i}+y_{i} \xi_{i}\right)\left(y_{j}+y_{j} \xi_{j}\right) \\
& +f_{i j}\left[y_{1}+y_{1} \xi_{1}(y), \ldots, y_{n}+y_{n} \xi_{n}(y)\right]\left(y_{i}+y_{i} \xi_{i}\right)\left(y_{j}+y_{j} \xi_{j}\right) .
\end{aligned}
$$

Les relations entres $\xi, f$ et $\psi$ s'obtiennent en égalisant les systèmes (6.16) et (6.17) :

$$
\begin{aligned}
a_{i j} & \left(y_{i}+y_{i} \xi_{i}\right)\left(y_{j}+y_{j} \xi_{j}\right) \\
& +f_{i j}\left[y_{1}+y_{1} \xi_{1}(y), \ldots, y_{n}+y_{n} \xi_{n}(y)\right]\left(y_{i}+y_{i} \xi_{i}\right)\left(y_{j}+y_{j} \xi_{j}\right) \\
= & \left\{y_{i}, y_{j}\right\}_{\Pi_{2}}+\left\{y_{i} \xi_{i}, y_{j}\right\}_{\Pi_{2}}+\left\{y_{i}, y_{j} \xi_{j}\right\}_{\Pi_{2}}+\left\{y_{i} \xi_{i}, y_{j} \xi_{j}\right\}_{\Pi_{2}} \\
& +\left\{y_{i}, y_{j}\right\}_{\psi}+\left\{y_{i} \xi_{i}, y_{j}\right\}_{\psi}+\left\{y_{i}, y_{j} \xi_{j}\right\}_{\psi}+\left\{y_{i} \xi_{i}, y_{j} \xi_{j}\right\}_{\psi} .
\end{aligned}
$$

0-Prénormalisation formelle. - Dans le but d'obtenir ultérieurement une série majorante on donne des conditions nécessaires et suffisantes pour que le changement de variable $x=y+\xi(y)$ effectue une 0 -prénormalisation de la structure de Poisson $\Pi$. La question de l'existence de changements de variables 0-prénormalisantes a déjà fait l'objet du lemme 6.1. Par définition, le changement de variable $x=y+\xi(y)$ effectue une 0 -prénormalisation de $\Pi$ si et seulement si pour tout $i<j$ on a $y_{i} y_{j} \Psi_{i j} \in \mathcal{J}$. Il suit que la propriété que $\Pi$ soit 0 -prénormalisé à l'ordre $m$ se traduit pour tout $i<j$ et $|Q|<m$ par la relation

$$
y_{i} y_{j} \Psi_{i j, Q} y^{Q} \in \mathcal{J}
$$

Pour tout multiindice $Q$ on définit $I_{Q}:=\left\{(i, j) \mid 1 \leq i<j \leq n, y_{i} y_{j} y^{Q} \notin \mathcal{J}\right\}$. Soient $\sum_{Q, i<j} a_{i j, Q} y_{i} y_{j} y^{Q}$ une série formelle, $Q_{0}$ un multiindice et $1 \leq i_{0}, j_{0} \leq n$. On note par $\left[\sum_{Q, i<j} a_{i j, Q} y_{i} y_{j} y^{Q}\right]_{i j Q_{0}}$ le terme $a_{i j, Q_{0}} y_{i_{0}} y_{j_{0}} y^{Q_{0}}$.

Lemme 6.7. - Supposons que le changement de variable $x=y+\xi^{m}$, définie par $x_{i}=y_{i}+\sum_{|Q|<m} y_{i} \xi_{i, Q} y^{Q}$, effectue une 0-prénormalisation de $\Pi \grave{a}$ l'ordre $m$. Alors le changement de variable $x=y+\xi^{m}+(\xi)_{m+1},(\xi)_{m+1}=$ $\left(y_{i} \sum_{|Q|=m} \xi_{i, Q} y^{Q}\right)_{i=1, \ldots, n}$, de la forme $x_{i}=y_{i}+\sum_{|Q|<m+1} y_{i} \xi_{i, Q} y^{Q}$, effectue une 0 -prénormalisation de $\Pi$ à l'ordre $m+1$ si et seulement si, pour tout $\left|Q_{0}\right|=m$ 
et tout $(i, j) \in I_{Q_{0}}$, on a

$$
\begin{aligned}
& {\left[a_{i j}\left(y_{i}+y_{i} \xi_{i}\right)\left(y_{j}+y_{j} \xi_{j}\right)\right.} \\
& \left.\quad+f_{i j}\left[y_{1}+y_{1} \xi_{1}(y), \ldots, y_{n}+y_{n} \xi_{n}(y)\right]\left(y_{i}+y_{i} \xi_{i}\right)\left(y_{j}+y_{j} \xi_{j}\right)\right]_{i j, Q_{0}} \\
& =\left[\left\{y_{i}, y_{j}\right\}_{\Pi_{2}}+\left\{y_{i} \xi_{i}, y_{j}\right\}_{\Pi_{2}}+\left\{y_{i}, y_{j} \xi_{j}\right\}_{\Pi_{2}}\right. \\
& \left.\quad+\left\{y_{i} \xi_{i}, y_{j} \xi_{j}\right\}_{\Pi_{2}}\right]_{i j, Q_{0}} .
\end{aligned}
$$

Preuve du lemme 6.\%. - Soit $\left|Q_{0}\right|=m$ fixé et $(\xi)_{m+1}$ quelconque. Par hypothèse, le changement de variable $x=y+\xi^{m}$ effectue une 0 -prénormalisation de $\Pi$ à l'ordre $m$. Par (6.19), les termes de degré $m+2$ des crochets $\left\{y_{i} \xi_{i}, y_{j}\right\}_{\psi}$, $\left\{y_{i}, y_{j} \xi_{j}\right\}_{\psi}$ et $\left\{y_{i} \xi_{i}, y_{j} \xi_{j}\right\}_{\psi}$ appartiennent à $\mathcal{J}$.

Toujours d'après (6.19), le changement de variable $x=y+\xi^{m}+(\xi)_{m+1}$ effectue alors une 0-prénormalisation à l'ordre $m+1$ de $\Pi$ si et seulement si pour tout $i<j$ le terme de degré $m+2$ du crochet $\left\{y_{i}, y_{j}\right\}_{\psi}$ appartient à $\mathcal{J}$. Pour ceci, d'après (6.18), il est nécessaire et suffisant que pour tout multiindice $\left|Q_{0}\right|=m$ et tout $(i, j) \in I_{Q_{0}}$ on a la relation (6.20).

Poursuivons la preuve du lemme 6.6. Rappelons (cf. (6.5)) qu'on a définie pour tout multiindice $Q$ l'ensemble $E_{Q} \subset\{1, \ldots, n\}$, tel que $i \in E_{Q}$ si et seulement si $y_{i} y^{Q} \notin \mathcal{J}$. Remarquons que le lemme 6.7 n'impose aucune condition sur les $\xi_{i, Q}$ tels que $i \notin E_{Q}$, i.e. $y_{i} y^{Q} \in \mathcal{J}$. En effectuant une récurrence, on déduit alors du lemme 6.7, le lemme suivant :

LEMME 6.8. - Le changement de variable formel $x_{i}=y_{i}+y_{i} \sum_{Q} \xi_{i, Q} y^{Q}$ effectue une 0-prénormalisation de $\Pi$ si et seulement si pour chaque multiindice $Q$ et chaque $i_{0} \in E_{Q}$ fixés, les nombres complexes $\xi_{i_{0}, Q}$ vérifient pour tout $\left(i_{0}, j\right) \in I_{Q}$ la relation (6.20). De plus, ce changement de variable peut être choisi tel que pour tout multiindice $Q$ et tout $i \notin E_{Q}$ on a $\xi_{i, Q}=0$.

Développons (6.20). Commençons par $\left\{y_{i} \xi_{i}, y_{j}\right\}_{\Pi^{2}}+\left\{y_{i}, y_{j} \xi_{j}\right\}_{\Pi^{2}}$. On a

$$
\begin{aligned}
\left\{y_{i} \xi_{i}, y_{j}\right\}_{\Pi^{2}} & =\left\{\sum_{|Q|>|R|} \xi_{i, Q} y_{i} y^{Q}, y_{j}\right\}_{\Pi^{2}} \\
& =\sum_{|Q|>|R|}\left\{\xi_{i, Q} y_{i} y^{Q}, y_{j}\right\}_{\Pi^{2}} .
\end{aligned}
$$

Rappelons que si $h$ et $g$ sont des fonctions analytiques, on a

$$
\{g, h\}_{\Pi^{2}}=\frac{1}{2}\left(\mathcal{L}_{X_{\Pi^{2}}(g)}(h)-\mathcal{L}_{X_{\Pi^{2}}(h)}(g)\right) .
$$


Rappelons également $\Pi^{2}=\frac{1}{2} \sum_{i \neq j} a_{i j} Y_{i} \wedge Y_{j}$, avec $a_{i j}=-a_{j i}$, et $A_{i}:=$ $\sum_{j=1}^{n} a_{i j} Y_{j}$. Or, si on pose $Q:=\left(Q_{1}, \ldots, Q_{l}\right)$, on a $X_{\Pi^{2}}\left(y_{j}\right)=y_{j} A_{j}$ et $X_{\Pi^{2}}\left(y_{i} y^{Q}\right)=y_{i} y^{Q}\left(A_{i}+\sum_{l=1}^{n} Q_{l} A_{l}\right)$. Donc, d'après (6.22)

$$
\begin{aligned}
\left\{\xi_{i, Q} y_{i} y^{Q}, y_{j}\right\}_{\Pi^{2}}= & \frac{1}{2} y_{i} y^{Q} \xi_{i, Q}\left(\mathcal{L}_{A_{i}}\left(y_{j}\right)+\sum_{l=1}^{n} Q_{l} \mathcal{L}_{A_{l}}\left(y_{j}\right)\right)-\frac{1}{2} \xi_{i, Q} y_{j} \mathcal{L}_{A_{j}}\left(y_{i} y^{Q}\right) \\
= & \frac{1}{2} y_{i} y^{Q} \xi_{i, Q}\left(a_{i j} y_{j}+\sum_{l=1}^{n} Q_{l} a_{l j} y_{j}\right) \\
& -\frac{1}{2} y_{j} y_{i} y^{Q} \xi_{i, Q}\left(a_{j i}+\sum_{l=1}^{n} Q_{i} a_{j i}\right) \\
= & -\xi_{i, Q} y_{i} y_{j} y^{Q} A_{j} \cdot Q+\xi_{i, Q} y_{i} y_{j} y^{Q} a_{i j} .
\end{aligned}
$$

Avec (6.21), on obtient

$$
\begin{aligned}
& \left\{y_{i} \xi_{i}, y_{j}\right\}_{\Pi^{2}}=\sum_{|Q|>|R|}\left(-y_{i} y_{j} \xi_{i, Q} y^{Q} A_{j} \cdot Q+\xi_{i, Q} y_{i} y_{j} y^{Q} a_{i j}\right) \\
& \left\{y_{i}, y_{j} \xi_{j}\right\}_{\Pi^{2}}=\sum_{|Q|>|R|}\left(y_{i} y_{j} \xi_{j, Q} y^{Q} A_{i} \cdot Q-\xi_{j, Q} y_{i} y_{j} y^{Q} a_{j i}\right) .
\end{aligned}
$$

D'où finalement

$$
\begin{aligned}
\left\{y_{i} \xi_{i}, y_{j}\right\}_{\Pi^{2}}+\left\{y_{i}, y_{j} \xi_{j}\right\}_{\Pi^{2}} & =\sum_{|Q|>|R|}\left(y_{i} y_{j} \xi_{j, Q} y^{Q} A_{i} \cdot Q-y_{i} y_{j} \xi_{i, Q} y^{Q} A_{j} \cdot Q\right) \\
& +\sum_{|Q|>|R|} \xi_{i, Q} y_{i} y_{j} y^{Q} a_{i j}-\sum_{|Q|>|R|} \xi_{j, Q} y_{i} y_{j} y^{Q} a_{j i} .
\end{aligned}
$$

Fixons maintenant $Q$. On a, comme $a_{i j}=-a_{j i}$,

$\left[a_{i j}\left(y_{i}+y_{i} \xi_{i}\right)\left(y_{j}+y_{j} \xi_{j}\right)\right]_{i j, Q}=a_{i j} \xi_{i, Q} y_{i} y_{j} y^{Q}-a_{j i} \xi_{j, Q} y_{i} y_{j} y^{Q}+\left[a_{i j} y_{i} \xi_{i} y_{j} \xi_{j}\right]_{i j, Q}$.

En remplaçant par (6.23) et par (6.24), la relation (6.20) devient

$$
\begin{aligned}
& {\left[a_{i j} y_{i} \xi_{i} y_{j} \xi_{j}-\left\{y_{i} \xi_{i}, y_{j} \xi_{j}\right\}_{\Pi_{2}}+\left[f _ { i j } \left[y_{1}+y_{1} \xi_{1}(y), \ldots\right.\right.\right.} \\
& \left.\left.y_{n}+y_{n} \xi_{n}(y)\right]\left(y_{i}+y_{i} \xi_{i}\right)\left(y_{j}+y_{j} \xi_{j}\right)\right]_{i j, Q} y_{i} y_{j} y^{Q}\left(\xi_{j, Q} A_{i} \cdot Q-\xi_{i, Q} A_{j} \cdot Q\right),
\end{aligned}
$$

$\operatorname{pour}(i, j) \in I_{Q}$.

Domination du premier membre de (6.25). - Soit $x=y+\xi$ un changement de coordonnées formel 0-prénormalisant $\Pi$ tel que dans le lemme 6.8, c'est-à-dire tel qu'en posant $\xi_{Q}:=\left(\xi_{i, Q} y_{i} y^{Q}\right)_{i=1, \ldots, n}$ pour tout $Q$, on ait $\xi_{i, Q}=0$ si $i \in E_{Q}$. 
Soit $Q$ un multiindice fixé. Définissons pour $(i, j) \in I_{Q}$ les nombres $D_{i j, Q} \in \mathbb{C}$ par

$$
\begin{aligned}
x^{Q} y_{i} y_{j} D_{i j, Q} & :=\left[a_{i j} y_{i} \xi_{i} y_{j} \xi_{j}-\left\{y_{i} \xi_{i}, y_{j} \xi_{j}\right\}_{\Pi_{2}}+\right. \\
& {\left[f_{i j}\left[y_{1}+y_{1} \xi_{1}(y), \ldots, y_{n}+y_{n} \xi_{n}(y)\right]\left(y_{i}+y_{i} \xi_{i}\right)\left(y_{j}+y_{j} \xi_{j}\right)\right]_{i j, Q} . }
\end{aligned}
$$

Il suit de [5], page 85, formule 1 , que si pour $(i, j) \in I_{Q}$ les relations (6.25) sont satisfaites, alors il existe un champ de bivecteurs quadratique $\Pi^{\prime}\left(\xi_{Q}\right)$ s'écrivant $\sum_{\substack{k \notin E_{Q} \\ i \neq k}} c_{i k} Y_{i} \wedge Y_{k}$ avec

$$
\left[\Pi^{2}, \xi_{Q}\right]=x^{Q} \sum_{\substack{i<j \\(i, j) \in I_{Q}}} D_{i j, Q} Y_{i} \wedge Y_{j}+x^{Q} \Pi^{\prime}\left(\xi_{Q}\right) .
$$

Autrement dit, $\xi$ vérifie propriété $(\mathrm{P})(\mathrm{cf} .(6.6))$ pour $I=Q$ et la notation $\Pi^{Q}=\sum_{i<j} D_{i j, Q} Y_{i} \wedge Y_{j}$. Pour pouvoir appliquer les lemmes 6.3-6.5, posons $D_{j i, Q}=-D_{i j, Q}$. On a ainsi $\Pi^{Q}=\frac{1}{2} \sum_{i \neq j} D_{i j, Q} Y_{i} \wedge Y_{j}$. Notons $\delta_{Q}^{\prime}=: \frac{|Q|}{n}\left(\left|\beta^{Q}\right|\right)$, où $\left|\beta^{Q}\right|$ est tel que dans le lemme 6.3. D'après le lemme 6.3 et le lemme 6.4, il est possible de choisir $\xi_{Q}$ tel que pour $i_{0} \in E_{Q}$ on a les majorations (6.9) ou (6.10) qui s'écrivent ici

$$
\left|\xi_{i_{0}, Q}\right| \leq \frac{1}{\delta_{Q}^{\prime}} \max _{\substack{i_{0} \neq j \\\left(i_{0}, j\right) \in I_{Q}}}\left(\left|D_{j i_{0}, Q}\right|\right)
$$

Introduisons quelques notations :

- si $\Phi=\sum_{I} \Phi_{I} y^{I}$ est une série formelle, nous notons $\bar{\Phi}=\sum_{I}\left|\Phi_{I}\right| y^{I}$

- si $\eta=\sum_{I} \eta_{I} y^{I}$, la notation $\eta \prec \Phi$ signifie que pour tout multiindice $I$ on a $\left|\eta_{I}\right|<\left|\Phi_{I}\right|$.

On a

$$
\left[\left\{y_{i} \xi_{i}, y_{j} \xi_{j}\right\}_{\Pi^{2}}\right]_{i j, Q}=\sum_{Q^{\prime}+Q^{\prime \prime}=Q}\left\{y_{i} y^{Q^{\prime}} \xi_{i, Q^{\prime}}, y_{j} y^{Q^{\prime \prime}} \xi_{j, Q^{\prime \prime}}\right\}_{\Pi^{2}} .
$$

On vérifie la relation de domination suivante

$$
\begin{aligned}
& \left\{y_{i} y^{Q^{\prime}} \xi_{i, Q^{\prime}}, y_{j} y^{Q^{\prime \prime}} \xi_{j, Q "}\right\}_{\Pi^{2}} \prec n\left(\max _{i \neq j}\left|a_{i j}\right|\right)\left(\max \left(\left|Q^{\prime}\right|+1,\left|Q^{\prime \prime}\right|+1\right)\right) \\
& \times\left|\xi_{i, Q^{\prime}} \xi_{j, Q},\right| y_{i} y_{j} y^{Q} \\
& \prec n\left(\max _{i \neq j}\left|a_{i j}\right|\right)(|Q|+1)\left|\xi_{i, Q^{\prime}} \xi_{j, Q}\right| y_{i} y_{j} y^{Q},
\end{aligned}
$$


où on rappelle que les $a_{i j}$ sont définies par la relation $\Pi^{2}=\frac{1}{2} \sum_{i \neq j} a_{i j} Y_{i} \wedge Y_{j}$, $a_{i j}=-a_{j i}$. Les relations (6.27) et (6.28) donnent

$$
\sum_{Q^{\prime}+Q^{\prime \prime}=Q}\left\{y_{i} y^{Q^{\prime}} \xi_{i, Q^{\prime}}, y_{j} y^{Q "} \xi_{j, Q}\right\}_{\Pi^{2}} \prec C|Q|\left[y_{i} \bar{\xi}_{i} y_{j} \bar{\xi}_{j}\right]_{i j Q},
$$

où $C=n \max _{i \neq j}\left|a_{i j}\right|+\tau$, avec $\tau \in \mathbb{R}^{+}$adéquat. Le premier membre de (6.25) vérifie alors

$$
\begin{aligned}
& {\left[a_{i j} y_{i} \xi_{i} y_{j} \xi_{j}-\left\{y_{i} \xi_{i}, y_{j} \xi_{j}\right\}_{\Pi_{2}}+\left[f _ { i j } \left[y_{1}+y_{1} \xi_{1}(y), \ldots,\right.\right.\right.} \\
& \left.\left.\quad y_{n}+y_{n} \xi_{n}(y)\right]\left(y_{i}+y_{i} \xi_{i}\right)\left(y_{j}+y_{j} \xi_{j}\right)\right]_{i j, Q} \\
& \prec C|Q|^{n+1}\left[\left|a_{i j}\right| y_{i} \bar{\xi}_{i} y_{j} \bar{\xi}_{j}+y_{i} \bar{\xi}_{i} y_{j} \bar{\xi}_{j}+\left[\overline { f } _ { i j } \left[y_{1}+y_{1} \bar{\xi}_{1}(y), \ldots,\right.\right.\right. \\
& \left.\left.\quad y_{n}+y_{n} \bar{\xi}_{n}(y)\right]\left(y_{i}+y_{i} \bar{\xi}_{i}\right)\left(y_{j}+y_{j} \bar{\xi}_{j}\right)\right]_{i j, Q} .
\end{aligned}
$$

On définit alors, pour $i<j,(i, j) \in I_{Q}$, les nombres $\bar{D}_{i j, Q} \in \mathbb{C}$ par les relations

$$
\begin{aligned}
y_{i} y_{j} y^{Q} \bar{D}_{i j, Q}=\left[\left|a_{i j}\right| y_{i} \bar{\xi}_{i} y_{j} \bar{\xi}_{j}+y_{i} \bar{\xi}_{i} y_{j} \bar{\xi}_{j}+\left[\overline { f } _ { i j } \left[y_{1}+y_{1} \bar{\xi}_{1}(y), \ldots,\right.\right.\right. \\
\left.\left.y_{n}+y_{n} \bar{\xi}_{n}(y)\right]\left(y_{i}+y_{i} \bar{\xi}_{i}\right)\left(y_{j}+y_{j} \bar{\xi}_{j}\right)\right]_{i j, Q}
\end{aligned}
$$

et posons $\bar{D}_{j i, Q}=-\bar{D}_{i j, Q}$. Comme par définition on a pour tout $1 \leq i \neq j \leq n$ la majoration $\left|D_{i j, Q}\right| \leq \bar{D}_{i j, Q}$, il suit de (6.26) que pour $i_{0} \in E_{Q}$ fixé,

$$
\left|\xi_{i, Q}\right| \leq \frac{C|Q|}{\delta_{Q}^{\prime}} \max _{\substack{i_{0} \neq j \\\left(i_{0}, j\right) \in I_{Q}}}\left(\bar{D}_{j i_{0}}\right)
$$

En posant $\delta_{Q}^{-1}=C|Q| \delta_{Q}^{\prime}-1$ la relation (6.30) devient

$$
\delta_{Q}\left|\xi_{i_{0}, Q}\right| \leq \max _{\substack{i_{0} \neq j \\\left(i_{0}, j\right) \in I_{Q}}}\left(\bar{D}_{j i_{0}}\right)
$$

Série majorante. - Comme les fonctions $f_{i j}$ sont analytiques et d'ordre $>|R|$, il existe deux constantes $a^{\prime}>0$ et $b^{\prime}>0$ telles que pour tout $i<j$ on a

$$
\bar{f}_{i j}\left[x_{1}, \ldots, x_{n}\right] x_{i} x_{j} \prec \frac{a^{\prime}\left(\sum_{j=1}^{n} x_{j}\right)^{2}}{1-b^{\prime}\left(\sum_{j=1}^{n} x_{j}\right)} .
$$

Puisqu'on a affaire à des séries à termes positifs, on en déduit

$$
\begin{aligned}
& {\left[\bar{f}_{i j}\left[y_{1}+y_{1} \bar{\xi}_{1}(y), \ldots, y_{n}+y_{n} \bar{\xi}_{n}(y)\right]\left(y_{i}+y_{i} \bar{\xi}_{i}\right)\left(y_{j}+y_{j} \bar{\xi}_{j}\right)\right]_{i j, Q} } \\
\prec & {\left[\frac{a^{\prime}\left(\sum_{j=1}^{n} y_{j}+\bar{\xi}_{j}\right)^{2}}{1-b^{\prime}\left(\sum_{j=1}^{n} y_{j}+\bar{\xi}_{j}\right)^{2}}\right]_{i j, Q} . }
\end{aligned}
$$

BUlletin DE LA SOCiÉtÉ MATHÉmATiQUe DE FRANCE 
qui nous fournit deux constantes $a, b>0$ telles que pour tout $i<j$ on ait

$$
\begin{aligned}
& {\left[\left|a_{i j}\right| y_{i} \bar{\xi}_{i} y_{j} \bar{\xi}_{j}+y_{i} \bar{\xi}_{i} y_{j} \bar{\xi}_{j}+\bar{f}_{i j}\left[y_{1}+y_{1} \bar{\xi}_{1}(y), \ldots,\right.\right.} \\
& \left.\left.\quad y_{n}+y_{n} \bar{\xi}_{n}(y)\right]\left(y_{i}+y_{i} \bar{\xi}_{i}\right)\left(y_{j}+y_{j} \bar{\xi}_{j}\right)\right]_{i j, Q} \\
& \prec\left[\frac{a\left(\sum_{j=1}^{n} y_{j}+\bar{\xi}_{j}\right)^{2}}{1-b\left(\sum_{j=1}^{n} y_{j}+\bar{\xi}_{j}\right)^{2}}\right]_{i j, Q},
\end{aligned}
$$

où $\bar{\xi}:=\sum_{i=1}^{n} \bar{\xi}_{i}$. Pour une série formelle quelconque $A=\sum_{Q, i=1}^{n} a_{Q, i} y^{i} y^{Q}$, notons $\{A\}_{Q}=\max _{1 \leq i \leq n}\left|a_{Q, i}\right|$. Comme on choisit pour tout multiindice $Q$ les termes $\xi_{Q}$ tels que relation (6.26) soit satisfaite, on déduit de (6.31) la majoration

$$
\delta_{Q}\left|\xi_{i, Q}\right| \leq\left\{\frac{a\left(\sum_{j=1}^{n} y_{j}+\bar{\xi}\right)^{2}}{1-b\left(\sum_{j=1}^{n} y_{j}+\bar{\xi}\right)^{2}}\right\}_{Q}
$$

pour $|Q|>|R|$ et $y_{i} y^{Q} \notin \mathcal{J}$. On définit alors la série formelle $\sigma(y)=$ $\sum_{|Q|>|R|} \sigma_{i, Q} y_{i} y^{Q}$ comme suit :

- si $i \in E_{Q}$, c'est-à-dire si $y_{i} y^{Q} \notin \mathcal{J}$, posons

$$
\sigma_{i, Q}=\left(\frac{a\left(\sum_{j=1}^{n} y_{j}+\sigma\right)^{2}}{1-b\left(\sum_{j=1}^{n} y_{j}+\sigma\right)^{2}}\right)_{i, Q} ;
$$

- si $i \notin E_{Q}$, c'est-à-dire si $y_{i} y^{Q} \in \mathcal{J}$, posons $\sigma_{i, Q}=0$.

Par la démarche utilisée pour établir le lemme 2.1 dans [15] p.1406-p.1407 nous allons déduire que la série $\sigma$ est convergente au voisinage de $0 \in \mathbb{C}^{n}$ :

Pour $|Q|=1$ on pose $\tilde{\eta}_{Q}=1$ et on définit pour $|Q|>1$ les nombres $\tilde{\eta}_{Q}$ par récurrence avec la relation

$$
\delta_{Q} \tilde{\eta}_{Q}=\max _{Q_{k}+\cdots+Q_{p}+S=Q} \tilde{\eta}_{Q_{1}} \cdots \tilde{\eta}_{Q_{p}}
$$

le maximum étant pris sur les ensembles de multiindices tels que $\left|Q_{i}\right|>|R|$ et $|S| \geq 0$. En utilisant (6.30),(6.31), (6.32) et (6.33) on déduit alors comme dans la preuve du lemme 2.2 dans [15] p.1409-p.1411 le résultat suivant.

Lemme 6.9. - Soit $|Q|>|R|$ tel que $x^{Q} \notin \mathcal{J}$. Soit $1 \leq i \leq n$. Alors on a $\left|\xi_{i, Q}\right| \leq \sigma_{\left(y_{i} Q\right)} \tilde{\eta}_{Q}$.

Le lemme 6.6 est donc une conséquence du lemme 6.10 ci-dessous.

LEMME 6.10. - Il existe $c>0$, tel que $\tilde{\eta}_{Q} \leq c^{|Q|}$. 
Preuve du lemme 6.10. - Soit $Q$ un multiindice (avec éventuellement une composante égale à -1$)$. Rappelons que $\left|\beta_{Q}\right|=\max _{1 \leq i \leq n}\left|\beta_{i}\right|$, où $\beta_{i}$ tel que $\mathcal{L}_{S_{i}}\left(x^{Q}\right)=\beta_{i} x^{Q}$. Définissons alors la suite $\left\{\eta_{Q}\right\}_{Q \in \mathcal{I}}$, où $\mathcal{I}=\left\{Q \mid x^{Q} \notin \mathcal{J}\right\}$ par

$$
\left|\beta_{Q}\right| \eta_{Q}=\max _{Q_{k}+\cdots+Q_{p}+S=Q} \eta_{Q_{1}} \cdots \eta_{Q_{p}},
$$

Rappelons que par définition,

$$
\delta_{Q}=C|Q| \delta_{Q}^{\prime}=C|Q|^{2}\left|\beta_{Q}\right| .
$$

Par définition, $\eta_{Q}$ est un produit de $1 /\left|\beta_{Q^{\prime}}\right|$ avec $\left|Q^{\prime}\right|>|Q|$. Soit $k \in \mathbb{N}^{*}$. Définissons $\Phi^{(k)}(Q)$ comme le nombre de $1 /\left|\beta_{Q^{\prime}}\right|$, avec $0 \neq d_{Q^{\prime}}<\omega_{k}$, qui sont présents dans ce produit, où $\omega_{k}$ tel que dans la preuve du lemme 6.5. Par des raisonnements identiques à celles qui permettent d'établir la proposition 2.2 (Estimation du nombre de petits diviseurs) dans [15] (cf. [15] section 2.2) on montre que pour tout $|Q|>R$ avec $x^{Q} \notin \mathcal{J}$, on a $\Phi^{(k)}(Q) \leq 2 n \frac{|Q|}{2^{k}}$. Soit $r$ entier et $2^{r}+1 \leq|Q|<2^{r+1}+1$. Par (6.34)

$$
\tilde{\eta}_{Q} \leq \prod_{k=0}^{r}\left(\frac{1}{C\left(2^{k+1}\right)^{2} \omega_{k+1}}\right)^{\Phi^{(k)}(Q)} .
$$

De ce qui précède on déduit

$$
\begin{aligned}
\ln \left(\tilde{\eta}_{Q}\right) & \leq-|Q| \sum_{k=0}^{r} \frac{1}{2^{k}} 2 n(2(k+1)) \ln 2-|Q| \sum_{k=0}^{r} \frac{2 n}{2^{k}} \ln \left(\omega_{k}\right) \\
& \leq|Q|\left(-\sum_{k \geq 0} \frac{1}{2^{k}} 2 n(2(k+1)) \ln 2-\sum_{k \geq 0} 2 n \frac{\ln \left(\omega_{k}\right)}{2^{k}}\right) .
\end{aligned}
$$

Par la condition diophantienne (6.11), la somme $\sum_{k \geq 0} 2 n \frac{\ln \left(\omega_{k}\right)}{2^{k}}$ converge, et on obtient finalement $\tilde{\eta}_{Q} \leq c^{|Q|}, c>0$.

Passage de la 0-prénormalisation à la prénormalisation. - Dans ce qui précède, on a établi que $\Pi$ pouvait être 0 -prénormalisé par une transformation analytique. On peut donc supposer que

$$
\Pi=\Pi^{2}+x^{R} \Pi^{R}+x^{R} \mathcal{R},
$$

où on note $\mathcal{R}$ un champ de bivecteurs analytique. Comme $R$ a toutes ses composantes strictement positives, on peut supposer

$$
\Pi=\Pi^{2}+x^{R} \Pi^{R}+\mathcal{R}^{\prime}, \quad \mathcal{R}^{\prime}=\sum_{J \in \mathbb{N}^{n}} x^{J} \Pi^{J},
$$

où on désigne par $\Pi^{J}$ des champs de bivecteurs quadratiques diagonaux. Les multiindices $J$ sont sans composante égale à -1 . Afin d'établir la proposition 
6.1, on veut montrer l'existence d'une prénormalisation, i.e. d'une transformation analytique $\Phi:\left(\mathbb{C}^{n}, 0\right) \rightarrow\left(\mathbb{C}^{n}, 0\right)$ telle que

$$
\Phi^{*}(\Pi)=\Pi^{2}+x^{R} \Pi^{R}+x^{R} \mathcal{R}, \quad \mathcal{R}=\sum_{J \in \mathbb{N}^{n}} x^{J} \Pi^{J} \in \mathfrak{J}_{1} .
$$

DÉFINITION 6.2. - On dit qu'une transformation formelle $\hat{\Phi}$ est diagonale si $\hat{\Phi}=\exp Z$, avec $Z=\sum_{I \in \mathbb{N}^{n}} c_{i}^{I} x^{I} Y_{i}, c \in \mathbb{C}$ (aucun multiindice apparaissant effectivement dans la somme n'a de composante égale à -1 ).

Lemme 6.11. - Supposons $\Pi$ tel que dans (6.35). Alors il existe des transformations formelles diagonaux $\hat{\Phi}$ prénormalisant $\Pi$.

La démonstration qui a permis de montrer le lemme 6.1 s'adapte pour montrer le lemme 6.11 : on suppose $\Pi$ 0-prénormalisé à l'ordre $\infty$ et prénormalisé à l'ordre $d$ et on montre l'existence d'une transformation diagonale $\Psi$ telle que $\Psi^{*}(\Pi)$ soit 0 -prénormalisé à l'ordre $\infty$ et prénormalisé à l'ordre $d+1$. Comme il n'y a plus de multiindice avec une composante égale à -1 il n'y a plus la difficulté de la preuve du lemme 6.2.

Le point de départ pour montrer l'existence d'une transformation holomorphe prénormalisante diagonale est le système suivant :

$$
\left\{x_{i}, x_{j}\right\}_{\Pi}=a_{i j} x_{i} x_{j}+f_{i j}(x) x_{i} x_{j}, 1 \leq i<j \leq n,
$$

où $f_{i j}$ est analytique (et non pas $f_{i j} x_{i} x_{j}$ comme pour la 0 -prénormalisation). La proposition 6.1 suit alors du lemme suivant.

LEMME 6.12. - Il existe un système de coordonnées locales analytique $x_{i}=$ $y_{i}+y_{i} \xi_{i}, 1 \leq i \leq n$ dans lequel le système (6.36) s'écrit sous la forme

$$
\left\{y_{i}, y_{j}\right\}_{\Pi}=a_{i j} y_{i} y_{j}+x^{R} g_{i j}(y) y_{i} y_{j}, 1 \leq i<\leq n
$$

où les $g_{i j}$ sont des fonction analytiques.

Le lemme 6.12 se démontre comme le lemme 6.6, sans toutefois les difficultés liées à la présence de multiindices avec une composante égale à -1 . Les adaptations dans la démonstration sont les suivantes :

- $x_{i}=y_{i}+y_{i} \xi_{i}$, avec $\xi_{i}=\sum_{Q \in \mathbb{N}^{n}} y^{Q}$;

- remplacer 0-prénormalisé par prénormalisé;

- remplacer $y_{i} y^{Q} \in \mathcal{J}$ (resp. $y_{i} y_{j} y^{Q} \in \mathcal{J}$ ) par $y^{Q} \in \mathcal{J}$, (et de même quand $i, j$ ou $Q$ est remplacé par une autre lettre);

- au lieu de demander $\left\{y_{i}, y_{j}\right\} \in \mathcal{J}$, demander $\left\{y_{i}, y_{j}\right\}=y_{i} y_{j} f$, où $f \in \mathcal{J}$, (resp. la même chose pour $\left\{\xi_{i} y_{i}, y_{j}\right\},\left\{y_{i}, \xi_{j} y_{j}\right\}$ et $\left\{\xi_{i} y_{i}, \xi_{j} y_{j}\right\}$ ).

TOME $137-2009-\mathrm{N}^{\mathrm{O}} 3$ 


\section{Champ de vecteurs associé}

Soit $\Pi=\sum_{I} x^{I} \Pi^{I}$ une structure de Poisson formelle. Soit $\Lambda:=\left(\lambda_{1}, \ldots, \lambda_{n}\right) \in$ $\mathbb{C}^{* n}$. La fonction $x^{\Lambda}$ n'a pas de détermination unique. Néanmoins le champ de vecteur $\frac{1}{x^{\Lambda}} X_{\Pi}\left(x^{\Lambda}\right)$ ne dépend pas du choix d'une détermination de $x^{\Lambda}$ : en effet, si on note $\Pi^{I}:=\frac{1}{2} \sum_{i=1}^{n} A_{i}^{I} \wedge Y_{i}$ où $A_{i}^{I}:=\sum_{j=1}^{n} a_{i j}^{I} Y_{j}$ avec $a_{i j}=-a_{j i}$, on a

$$
\frac{1}{x^{\Lambda}} X_{\Pi^{I}}\left(x^{\Lambda}\right)=\left(\sum_{i=1}^{n} \lambda_{i} A_{i}^{I}\right) .
$$

REMARQUE. - Soit $B$ un champ de bivecteurs quadratique diagonal. Alors $\frac{1}{x^{\Lambda}} X_{B}\left(x^{\Lambda}\right)$ est un champ de vecteurs linéaire diagonal. Si aucun multiindice apparaissant effectivement dans la somme $\Pi=\sum_{I} x^{I} \Pi^{I}$ possède une composante égale à -1 , le champ de vecteurs $\frac{1}{x^{\Lambda}} X_{\Pi}\left(x^{\Lambda}\right)$ est analytique en $0 \in \mathbb{C}^{n}$. Soient $\Pi$ et $\Pi^{\prime}$ deux champs de bivecteurs formels. On a

$$
\frac{1}{x^{\Lambda}} X_{\Pi+\Pi^{\prime}}\left(x^{\Lambda}\right)=\frac{1}{x^{\Lambda}} X_{\Pi}\left(x^{\Lambda}\right)+\frac{1}{x^{\Lambda}} X_{\Pi^{\prime}}\left(x^{\Lambda}\right) .
$$

On reprend la notion suivante, introduite par Stolovitch dans [19], sur une idée de Marc Chaperon.

DÉfinition 7.1. - Soit $\mathbb{S}=\operatorname{Vect}\left\langle S_{1}, S_{2}\right\rangle$ tel qu'en (P4), théorème 3.1. Alors $T \in \mathbb{S}$ est un élément de Cartan de $\mathbb{S}$ si l'ensemble des intégrales premières formelles de $T$ coïncide avec l'ensemble des intégrales premières formelles communes à tous les éléments de $\mathbb{S}$.

Proposition 7.1. - Soient $\Pi^{2}$ une structure de Poisson quadratique diagonale telle qu'en (P1), (P2), théorème 3.1, et $\mathbb{S}=\operatorname{Vect}\left\langle S_{1}, S_{2}\right\rangle$ tel qu'en (P4), théorème 3.1. Alors il existe une réunion dénombrable d'hyperplans de $\mathbb{C}^{n} A$ telle que pour $\Lambda \in \mathbb{C}^{n}-A$ le champ de vecteurs linéaire diagonal $\frac{1}{x^{\Lambda}} X_{\Pi^{2}}\left(x^{\Lambda}\right)$ est un élément de Cartan de $\mathbb{S}$.

En utilisant (7.1), la preuve se fait de manière identique à celle du lemme 3.1 dans [19], sur une idée de Marc Chaperon (voir aussi [3]).

Proposition 7.2. - Soit $\Pi$ une structure de Poisson telle qu'en la proposition 4.1, et soit $\mathbb{S}=\operatorname{Vect}\left\langle S_{1}, S_{2}\right\rangle$ (cf. (P4), théorème 3.1). Supposons $\Pi=$ $\sum_{I} x^{I} \Pi^{I}$ telle qu'aucun multiindice apparaissant effectivement dans la somme n'a une composante égale à -1 . Soit $\Lambda:=\left(\lambda_{1}, \ldots, \lambda_{n}\right) \in \mathbb{C}^{n}$ tel que le champ de vecteurs linéaire diagonal $\frac{1}{x^{\Lambda}} X_{\Pi^{2}}\left(x^{\Lambda}\right)$ soit un élément de Cartan de $\mathbb{S}$ tel que $\Lambda . S \neq 0$.

(i) Alors $\frac{1}{x^{\Lambda}} X_{\Pi}\left(x^{\Lambda}\right)$ est conjugué à la forme normale polynomiale $\frac{1}{x^{\Lambda}} X_{\left[\Pi^{2}+x^{R} \Pi^{R}\right]}\left(x^{\Lambda}\right)$ par une unique transformation formelle $\hat{\Phi}$. 
(ii) $\hat{\Phi}$ conjugue également $\Pi$ à la forme normale polynomiale $\Pi^{2}+x^{R} \Pi^{R}$. Preuve de la proposition 7.2 (i). - Posons

$$
\frac{1}{x^{\Lambda}} X_{\Pi}\left(x^{\Lambda}\right):=\frac{1}{x^{\Lambda}} X_{\Pi^{2}}\left(x^{\Lambda}\right)+\frac{1}{x^{\Lambda}} X_{x^{R} \Pi^{R}}\left(x^{\Lambda}\right)+\sum_{|I|>|R|} x^{I} \frac{1}{x^{\Lambda}} X_{\Pi^{I}}\left(x^{\Lambda}\right),
$$

où les champs de vecteurs $\frac{1}{x^{\Lambda}} X_{\Pi^{I}}\left(x^{\Lambda}\right)$ sont linéaires diagonaux.

Les intégrales premières formelles du champ de vecteurs linéaire diagonal sont exactement les intégrales premières formelles communes à $S_{1}$ et $S_{2}$. Ils coïncident donc avec les fonctions de Casimir formelles associés à la structure de Poisson $\Pi^{2}$. On en déduit que $\frac{1}{x^{\Lambda}} X_{\Pi^{2}}\left(x^{\Lambda}\right)$ est 1-résonnant, c'est-à-dire les termes résonnants (au sens de Poincaré-Dulac) de (7.3) sont de la forme $\left(x^{R}\right)^{d} D$, où $d$ est un entier strictement positif, et $D$ un champ de vecteurs linéaire diagonal.

En outre, rappelons que $\Pi^{R}:=S \wedge C$ (hypothèse $\left(P_{4}\right)$, théorème 3.1). Constatons alors que le champ de vecteurs $\frac{1}{x^{\Lambda}} X_{x^{R} \Pi^{R}}\left(x^{\Lambda}\right)=x^{R} \frac{1}{x^{\Lambda}}\left(\mathcal{L}_{S}\left(x^{\Lambda}\right) C-\right.$ $\mathcal{L}_{C}\left(x^{\Lambda}\right) S$ n'admet pas $x^{R}$ comme intégrale première formelle puisque d'une part par hypothèse sur $\Lambda$, on a $\Lambda . S=\frac{1}{x^{\Lambda}} X_{S}\left(x^{\Lambda}\right) \neq 0$, et d'autre part le champ $C$ n'admet par hypothèse pas $x^{R}$ comme intégrale première formelle (rappelons que $S \in \mathbb{S}$, donc $\left.\mathcal{L}_{S}\left(x^{R}\right)=0\right)$.

Supposons $\frac{1}{x^{\Lambda}} X_{\Pi}\left(x^{\Lambda}\right)$ formellement normalisé au degré $d$, i.e.

$$
\begin{aligned}
\frac{1}{x^{\Lambda}} X_{\Pi}\left(x^{\Lambda}\right)=\frac{1}{x^{\Lambda}} X_{\Pi^{2}}\left(x^{\Lambda}\right)+\frac{1}{x^{\Lambda}} X_{x^{R} \Pi^{R}}\left(x^{\Lambda}\right) & +\sum_{\mathcal{L}_{\left[\frac{1}{x^{\Lambda}} X_{\left.\Pi^{2}\left(x^{\Lambda}\right)\right]}\right]^{\left(x^{I}\right) \neq 0}}\left(x^{I}\right) \frac{1}{x^{\Lambda}} X_{\Pi^{I}}\left(x^{\Lambda}\right)} \\
& +\sum_{j|R| \geq d+|R|} x^{j R} \frac{1}{x^{\Lambda}} X_{\left[x^{j R} \Pi^{j R}\right]}\left(x^{\Lambda}\right) .
\end{aligned}
$$

D'après le procédé classique bien connu de normalisation de champs de vecteurs par récurrence sur le degré on aura établi 7.2 (i) si

- pour les multiindices $|I|=d$ tels que $\mathcal{L}_{\frac{1}{x^{\Lambda}} X_{\Pi^{2}}\left(x^{\Lambda}\right)}\left(x^{I}\right) \neq 0$ il existe un unique champ de vecteurs linéaire diagonal $Z^{I}$ qui satisfait l'équation cohomologique

$$
\left[\frac{1}{x^{\Lambda}} X_{\Pi^{2}}\left(x^{\Lambda}\right), x^{I} Z^{I}\right]=x^{I} \frac{1}{x^{\Lambda}} X_{\Pi^{I}}\left(x^{\Lambda}\right)
$$

(cas non-résonnant). L'existence et l'unicité des solutions de la dernière équation cohomologique est un fait bien connu.

- pour les multiindices $|I|=d$ tels que $\mathcal{L}_{\frac{1}{x^{\Lambda}} X_{\Pi^{2}}\left(x^{\Lambda}\right)}\left(x^{I}\right)=0$ il existe un unique champ de vecteurs linéaire diagonal qui vérifie l'équation

$$
\left[\frac{1}{x^{\Lambda}} X_{x^{R} \Pi^{R}}\left(x^{\Lambda}\right), x^{I} Z^{I}\right]=x^{I+R} \frac{1}{x^{\Lambda}} X_{\Pi^{I+R}}\left(x^{\Lambda}\right)
$$


(cas résonnant). L'existence et l'unicité des solutions $Z^{I}$ de (7.6) reste à montrer.

Puisque $\frac{1}{x^{\Lambda}} X_{\Pi^{2}}\left(x^{\Lambda}\right)$ est 1-résonnant, $x^{I}$ est de la forme $x^{j R}$, où $j \in \mathbb{N}^{*}$. Rappelons $\frac{1}{x^{\Lambda}} X_{x^{R} \Pi^{R}}\left(x^{\Lambda}\right)=x^{R} \frac{1}{x^{\Lambda}}\left(\mathcal{L}_{S}\left(x^{\Lambda}\right) C-\mathcal{L}_{C}\left(x^{\Lambda}\right) S\right)$. Posons $S . \Lambda:=$ $\frac{1}{x^{\Lambda}} \mathcal{L}_{S}\left(x^{\Lambda}\right)$ et $T:=\frac{1}{x^{\Lambda}} X_{\Pi^{R}}\left(x^{\Lambda}\right)$.

Comme $\frac{1}{x^{\Lambda}} X_{\Pi^{2}}\left(x^{\Lambda}\right)$ est 1-résonnant, il existe $j \in \mathbb{N}^{*}$ tel que $x^{I}=x^{j R}$. Par $\mathcal{L}_{S}\left(x^{R}\right)=0$ on a

$$
\mathcal{L}_{\frac{1}{x^{\Lambda}} X_{\Pi^{R}}\left(x^{\Lambda}\right)}\left(x^{R}\right)=S . \Lambda \mathcal{L}_{C}\left(x^{j R}\right) .
$$

D'où, pour tout champ de vecteurs linéaire diagonal $L$,

$$
\left[\frac{1}{x^{\Lambda}} X_{x^{R} \Pi^{R}}\left(x^{\Lambda}\right), x^{I} L\right]=\left[x^{R} T, x^{j R} L\right]=S . \Lambda x^{R} \mathcal{L}_{C}\left(x^{j R}\right) L-x^{I} \mathcal{L}_{L}\left(x^{R}\right) T .
$$

Soit $Z^{\prime}$ un champ de vecteurs linéaire diagonal tel que $S . \Lambda x^{R} \mathcal{L}_{C}\left(x^{j R}\right) Z^{\prime}=$ $\frac{1}{x^{\Lambda}} X_{x^{j R} \Pi^{j R}}\left(x^{\Lambda}\right)$. Montrons qu'il existe $c \in \mathbb{C}$ tel que $x^{j R} Z^{j R}=x^{j R} Z^{\prime}+c x^{j R} T$ convient comme unique solution de (7.6). - Existence : d'après (7.8), il suffit que

$$
\left[x^{R} T, c x^{j R} T\right]=x^{I} \mathcal{L}_{Z^{\prime}}\left(x^{R}\right) T,
$$

donc, toujours d'après (7.7) et (7.8),

$$
S . \Lambda x^{R} \mathcal{L}_{C}\left(x^{j R}\right) c T+x^{j R} \mathcal{L}_{[c S . \Lambda C]}\left(x^{R}\right) T=x^{I} \mathcal{L}_{Z^{\prime}}\left(x^{R}\right) T .
$$

Il est facile de voir l'existence d'un unique $c$ vérifiant la dernière égalité.

- Unicité : soit $x^{j R} Z$ une solution quelconque de (7.6). Le champ de vecteurs $x^{j R}\left(Z-Z^{\prime}\right)$ doit nécessairement vérifier l'équation (7.9) qui adment une solution unique.

Preuve de la proposition $\% .2$ (ii). - Montrons que $\hat{\Phi}$ conjugue $\Pi$ à la forme normale polynomiale $\Pi^{2}+x^{R} \Pi^{R}$.

Supposons $\Pi$ normalisé au degré $d$, i.e.

$$
\Pi=\Pi^{2}+x^{R} \Pi^{R}+\left(\sum_{\substack{|I|=d \\ X_{\Pi^{2}}\left(x^{I}\right) \neq 0}} x^{I} \Pi^{I}\right)+x^{j R} \Pi^{j R}+\mathfrak{R}
$$

où on a mis entre parenthèses la somme des termes non-résonnants, et où $|j R|=d+|R|$. Par $\mathfrak{R}$ on désigne la somme des terme non-résonnants d'ordre strictement supérieurs à $d$ et des termes résonnants d'ordre strictement supérieur à $d+|R|$ au niveau des termes résonnants. 
Le champ de vecteurs $\frac{1}{x^{\Lambda}} X_{\Pi}\left(x^{\Lambda}\right)$ s'écrit $\frac{1}{x^{\Lambda}} X_{\Pi}\left(x^{\Lambda}\right)=\frac{1}{x^{\Lambda}} X_{\left[\Pi^{2}+x^{R} \Pi^{R}\right]}\left(x^{\Lambda}\right)+\left(\sum_{|I|=d} x^{I} \frac{1}{x^{\Lambda}} X_{\Pi^{I}}\left(x^{\Lambda}\right)\right)+x^{j R} \frac{1}{x^{\Lambda}} X_{\Pi^{j R}}\left(x^{\Lambda}\right)+\mathfrak{R}^{\prime}$.

On déduit de la proposition 7.2 (i) que le champ de vecteurs $\frac{1}{x^{\Lambda}} X_{\Pi}\left(x^{\Lambda}\right)$ se normalise au degré $d+1$ (au sens (7.4)) par le bias d'une unique transformation de la forme $\exp \left(-\sum_{|J|=d} x^{J} Z^{J}\right)$, où $Z^{J}$ est un champ de vecteurs linéaire diagonal.

Comme $\frac{1}{x^{\Lambda}} X_{\Pi}\left(x^{\Lambda}\right)$ est parallèle au champ hamiltonien de $x^{\Lambda}$ par rapport à $\Pi$, on a $\mathcal{L}_{\frac{1}{x^{\Lambda}} X_{\Pi}\left(x^{\Lambda}\right)}\left(x^{\Lambda}\right)=0$. On en déduit que pour tout $|J|=d$, on a $\mathcal{L}_{Z^{J}}\left(x^{\Lambda}\right)=0$. D'où la relation

$$
\exp \left(x^{I} Z^{J}\right)^{*}\left(\frac{1}{x^{\Lambda}} X_{\Pi}\left(x^{\Lambda}\right)\right)=\frac{1}{x^{\Lambda}} X_{\left[\exp \left(x^{J} Z^{J}\right)^{*}(\Pi)\right]}\left(x^{\Lambda}\right) .
$$

On déduit de (7.11) que pour prouver la proposition 7.2 (ii) il suffit de montrer que $\exp \left(\sum_{|J|=d} x^{J} Z^{d}\right)$ normalise également $\Pi$ au degré $d+1$ (au sens (7.10)). La relation (7.11) permet en effet d'itérer du degré $|d|$ au degré $|d+1|$.

L'identité de Jacobi $[\Pi, \Pi]=0$ impose aux termes non-résonnants de degré $d$ de $\Pi$ de vérifier

$$
\left[\Pi^{2}, x^{I} \Pi^{I}\right]=0,
$$

et aux terme résonnant de degré $d+|R|$ de $\Pi$ de vérifier

$$
\left[x^{R} \Pi^{R}, x^{j R} \Pi^{j R}\right]=0 .
$$

On alors déduit des relations (7.5), (7.6), (7.12) et (7.13) que la proposition 7.2 (ii) est conséquence du lemme 7.1 plus bas.

Lemme 7.1. - (i) Soient $x^{I}$ tel que $X_{\Pi^{2}}\left(x^{I}\right) \neq 0$ i.e. tel que $\mathcal{L}_{\frac{1}{x^{\Lambda}} X_{\Pi^{2}}\left(x^{\Lambda}\right)}\left(x^{I}\right) \neq 0$, et $B$ un champ de bivecteurs quadratique diagonal non nul tel que $\left[\Pi^{2}, x^{I} B\right]=0$. Alors $\frac{1}{x^{\Lambda}} X_{x^{I} B}\left(x^{\Lambda}\right) \neq 0$ et tout champ de vecteurs linéaire diagonal $Z$ qui vérifie l'équation

$$
\left[x^{I} Z, \frac{1}{x^{\Lambda}} X_{\Pi^{2}}\left(x^{\Lambda}\right)\right]=\frac{1}{x^{\Lambda}} X_{x^{I} B}\left(x^{\Lambda}\right)
$$

vérifie également l'équation $\left[x^{I} Z, \Pi^{2}\right]=x^{I} B$.

(ii) Soit $j \geq 2$ un entier, et $A$ un champ de bivecteurs quadratique diagonal tel que $x^{(j+1) R} A$ vérifie l'équation $\left[x^{R} \Pi^{R}, x^{(j+1) R} A\right]=0$. Alors $\frac{1}{x^{\Lambda}} X_{x^{(j+1) R} A}\left(x^{\Lambda}\right) \neq 0$ et tout champ de vecteurs linéaire diagonal $Z^{\prime}$ vérifiant l'équation $\left[x^{j R} Z^{\prime}, \frac{1}{x^{\Lambda}} X_{x^{R} \Pi^{R}}\left(x^{\Lambda}\right)\right]=\frac{1}{x^{\Lambda}} X_{x^{(j+1) R} A}\left(x^{\Lambda}\right)$ vérifie également l'équation $\left[x^{j R} Z^{\prime}, x^{R} \Pi^{R}\right]=x^{(j+1) R} A$. 
Preuve du lemme $\% .1$ (i). - Comme $\left[\Pi^{2}, x^{I} B\right]=0$ et l'hypothèse $(\mathrm{H})$ est satisfaite, on montre comme dans la démonstration de la proposition 2.1 (cf. [5] section 3) qu'il existe un champ de vecteurs linéaire diagonal $T$ tel que

$$
x^{I} B=\left[\Pi^{2}, x^{I} T\right]=X_{\Pi^{2}}\left(x^{I}\right) \wedge T .
$$

Or, avec (2.6) on déduit de (7.15)

$$
\frac{1}{x^{\Lambda}} X_{x^{I} B}\left(x^{\Lambda}\right)=\frac{1}{x^{\Lambda}} \mathcal{L}_{\left[X_{\Pi^{2}}\left(x^{I}\right)\right]}\left(x^{\Lambda}\right) T-\frac{1}{x^{\Lambda}} \mathcal{L}_{T}\left(x^{\Lambda}\right) X_{\Pi^{2}}\left(x^{I}\right) .
$$

Montrons d'abord que si $\frac{1}{x^{\Lambda}} X_{\Pi^{2}}\left(x^{\Lambda}\right)$ est un élément de Cartan de $\mathbb{S}$ alors

$$
\frac{1}{x^{\Lambda}} \mathcal{L}_{\left[X_{\Pi^{2}}\left(x^{I}\right)\right]}\left(x^{\Lambda}\right) \neq 0 \text {. }
$$

Comme $B$ est non-nul, il suit de (7.15) que le champ linéaire diagonal $\frac{1}{x^{I}} X_{\Pi^{2}}\left(x^{I}\right)$ n'est pas colinéaire à $T,(7.16)$ et (7.17) impliquent alors la non-nullité de $\frac{1}{x^{\Lambda}} X_{x^{I} B}\left(x^{\Lambda}\right)$. Posons $I:=\left(I_{1}, \ldots, I_{n}\right)$. On note $\frac{1}{x^{I}} X_{\Pi^{2}}\left(x^{I}\right)=$ $\sum_{i=1}^{n} I_{i} S_{i}$. D'où

$$
\frac{1}{x^{\Lambda}} \mathcal{L}_{X_{\Pi^{2}}\left(x^{I}\right)}\left(x^{\Lambda}\right)=\sum_{j=1}^{n} \lambda_{i}\left(\sum_{i=1}^{n} I_{i} a_{i j}\right),
$$

(on rappelle que $S_{i}:=\sum_{j=1}^{n} a_{i j} Y_{j}$ ). Or,

$$
\sum_{j=1}^{n} \lambda_{i}\left(\sum_{i=1}^{n} I_{i} a_{i j}\right)=\sum_{1 \leq i, j \leq n}\left(I_{i} \lambda_{j}\right) a_{i j}=\sum_{i=1}^{n} I_{i}\left(\sum_{j=1}^{n} \lambda_{j} a_{i j}\right)=\mathcal{L}_{\left[\frac{1}{x^{\Lambda}} X_{\Pi^{2}}\left(x^{\Lambda}\right)\right]}\left(x^{I}\right) .
$$

De (7.18), (7.19) suit

$$
\frac{1}{x^{\Lambda}} \mathcal{L}_{\left[X_{\Pi^{2}}\left(x^{I}\right)\right]}\left(x^{\Lambda}\right)=\mathcal{L}_{\left[\frac{1}{x^{\Lambda}} X_{\Pi^{2}}\left(x^{\Lambda}\right)\right]}\left(x^{I}\right) .
$$

On en déduit $\mathcal{L}_{\left[\frac{1}{x^{\Lambda}} X_{\Pi^{2}}\left(x^{\Lambda}\right)\right]}\left(x^{I}\right) \neq 0$, puisque $x^{I}$ est non-résonnant et $\frac{1}{x^{\Lambda}} X_{\Pi^{2}}\left(x^{\Lambda}\right)$ est un élément de Cartan de $\mathbb{S}$.

On reprend la preuve du lemme 7.1 (i). Soit $Z$ un champ de vecteurs linéaire diagonal vérifiant (7.14). On a la relation $\left[\frac{1}{x^{\Lambda}} X_{\Pi^{I}}\left(x^{\Lambda}\right), x^{I} Z\right]=$ $\mathcal{L}_{\left[\frac{1}{x^{\Lambda}} X_{\Pi^{I}}\left(x^{\Lambda}\right)\right]}\left(x^{I}\right) Z$. On en déduit avec (7.16), (7.20), qu'il existe $s \in \mathbb{C}$ tel que $Z=T+s \frac{1}{x^{I}} X_{\Pi^{2}}\left(x^{I}\right)$. Rappelons $\left[\frac{1}{x^{I}} X_{\Pi^{2}}\left(x^{I}\right), \Pi^{2}\right]=0$. Par (7.15) on déduit finalement $\left[\Pi^{2}, Z\right]=x^{I} B$.

Preuve du lemme $\% .1$ (ii). - Commençons par montrer que le champ de vecteurs $\frac{1}{x^{\Lambda}} X_{\left[x^{(j+1) R} A\right]}\left(x^{\Lambda}\right)$ est non nul. Par hypothèse, on a $\left[x^{R} \Pi^{R}, x^{(j+1) R} A\right]=$ 
0 . D'après la proposition 4.3, ceci signifie qu'il existe un champ de vecteurs $Z$ linéaire diagonal tel que

$$
\left[\Pi^{R}, x^{j R} Z\right]=x^{j R} A .
$$

D'après le lemme 2.1, on a alors $x^{j R} A=X_{\Pi^{R}}\left(x^{j R}\right) \wedge Z$. Par hypothèse, on a $\Pi^{R}=S \wedge C$, ainsi que $\mathcal{L}_{S}\left(x^{j R}\right)=0$. D'où, par (2.6),

$$
x^{j R} A=-\mathcal{L}_{C}\left(x^{j R}\right) S \wedge Z .
$$

On en déduit

$$
X_{\left[x^{j R} A\right]}\left(x^{\Lambda}\right)=-\left(\mathcal{L}_{C}\left(x^{j R}\right)\right)\left(\mathcal{L}_{S}\left(x^{\Lambda}\right) Z-\mathcal{L}_{Z}\left(x^{\Lambda}\right) C\right) .
$$

Par hypothèse on a $\mathcal{L}_{C}\left(x^{R}\right) \neq 0$ et $\mathcal{L}_{S}\left(x^{\Lambda}\right) \neq 0$. Comme $A$ est non nul la relation (7.22) implique que $C$ et $Z$ ne sont pas colinéaires. Le champ de vecteurs $\frac{1}{x^{\Lambda}} X_{\left[x^{j R} A\right]}\left(x^{\Lambda}\right)$ n'est donc pas nul. Comme $\frac{1}{x^{\Lambda}} X_{\left[x^{(j+1) R} A\right]}\left(x^{\Lambda}\right)=$ $x^{R} \frac{1}{x^{\Lambda}} X_{\left[x^{j R} A\right]}\left(x^{\Lambda}\right)$ le champ de vecteurs $\frac{1}{x^{\Lambda}} X_{\left[x^{(j+1) R} A\right]}\left(x^{\Lambda}\right)$ est également non nul, ce qu'il fallait montrer.

On reprend la preuve du lemme 7.1 (ii). D'après la proposition 7.2 (i), il existe un champ linéaire diagonal $Z^{\prime}$ vérifiant l'équation

$$
\left[x^{j R} Z^{\prime}, x^{R} \frac{1}{x^{\Lambda}} X_{\Pi^{R}}\left(x^{\Lambda}\right)\right]=\frac{1}{x^{\Lambda}} X_{\left[x^{(j+1) R} A\right]}\left(x^{\Lambda}\right) .
$$

On a la relation

$$
\begin{aligned}
{\left[x^{j R}\left(Z^{\prime}\right),\right.} & \left.x^{R} \frac{1}{x^{\Lambda}} X_{\Pi^{R}}\left(x^{\Lambda}\right)\right] \\
& =x^{R}\left[x^{j R} Z^{\prime}, \frac{1}{x^{\Lambda}} X_{\Pi^{R}}\left(x^{\Lambda}\right)\right]+\mathcal{L}_{x^{j R} Z^{\prime}}\left(x^{R}\right)\left(\frac{1}{x^{\Lambda}} X_{\Pi^{R}}\left(x^{\Lambda}\right)\right) \\
& =x^{R}\left[x^{j R} Z^{\prime}, \frac{1}{x^{\Lambda}} X_{\Pi^{R}}\left(x^{\Lambda}\right)\right]+c x^{j R} x^{R}\left(\frac{1}{x^{\Lambda}} X_{\Pi^{R}}\left(x^{\Lambda}\right)\right) \\
& =\frac{1}{x^{\Lambda}} X_{\left[x^{(j+1) R} A\right]}\left(x^{\Lambda}\right),
\end{aligned}
$$

où on a posé $c \in \mathbb{C}$ tel que $c x^{j R} x^{R}=\mathcal{L}_{\left[x^{j} R Z^{\prime}\right]}\left(x^{R}\right)$. De plus, d'après (2.8)-(2.10), on a

$$
\begin{aligned}
{\left[x^{j R} Z^{\prime}, x^{R} \Pi^{R}\right] } & =x^{R}\left[x^{j R} Z^{\prime}, \Pi^{R}\right]+\mathcal{L}_{\left[x^{j R} Z^{\prime}\right]}\left(x^{R}\right) \Pi^{R} \\
& =x^{R}\left[x^{j R} Z^{\prime}, \Pi^{R}\right]+c x^{j R} x^{R} \Pi^{R}
\end{aligned}
$$

Soit $B$ tel que $x^{(j+1) R} A-c x^{j R} x^{R} \Pi^{R}=x^{(j+1) R} B$. D'après (7.26), le lemme 7.1 (ii) suit alors de

$$
\left[x^{j R} Z^{\prime}, \Pi^{R}\right]=x^{j R} B .
$$

TOME $137-2009-\mathrm{N}^{\mathrm{O}} 3$ 
Or, en divisant par $x^{R}$ on déduit de (7.25)

$$
\frac{1}{x^{\Lambda}} X_{\left[x^{j R} B\right]}\left(x^{\Lambda}\right)=\frac{1}{x^{\Lambda}} X_{\left[x^{j R} A\right]}\left(x^{\Lambda}\right)-c x^{j R} \frac{1}{x^{\Lambda}} X_{\Pi^{R}}\left(x^{\Lambda}\right) .
$$

La relation (7.25) signifie

$$
\left[x^{j R} Z^{\prime}, \frac{1}{x^{\Lambda}} X_{\Pi^{R}}\left(x^{\Lambda}\right)\right]=x^{j R} \frac{1}{x^{\Lambda}} X_{\left[x^{j R} B\right]}\left(x^{j R}\right) .
$$

Comme $\Pi^{R}$ est une structure de Poisson quadratique diagonale, le raisonnement qui a permis d'établir le lemme 7.1 (i) s'applique aussi si on remplace $\Pi^{2}$ par $\Pi^{R}$, et $x^{I}$ par $x^{j R}$. (Le fait que $\Lambda$ soit un élément de Cartan de $\mathbb{S}$ n'a servi dans la preuve du lemme 7.1 (i) uniquement pour assurer que les termes de (7.20) soient non nuls. Dans le cas présent, il est déjà établi dans le raisonnement qui suit (7.23) que $\mathcal{L}_{\left[\frac{1}{x^{\Lambda}} X_{\Pi^{R}}\left(x^{\Lambda}\right)\right]}\left(x^{j R}\right)$ est non nul.)

La relation (7.27) découle donc du lemme 7.1 (i) puisque

$$
\left[\Pi^{R}, x^{j R} B\right]=\left[\Pi^{R}, x^{j R} A\right]-\left[\Pi^{R}, c x^{j R} x^{R} \Pi^{R}\right]=0 .
$$

En effet comme $\Pi^{R}$ est produit extérieur de deux champs de vecteurs on a $\left[\Pi^{R}, c x^{j R} x^{R} \Pi^{R}\right]=0$. La nullité du crochet $\left[\Pi^{R}, x^{j R} A\right]$ suit alors de $(7.21)$ et $\mathrm{du}$ lemme 4.2 (iii).

\section{Normalisation sectorielle}

Rappel. - On donne sous une forme légèrement simplifiée le résultat de Stolovitch ([16], section 3.3) sur la normalisation sectorielle de champs de vecteurs.

DÉFINITION 8.1. - Un champ de vecteurs holomorphe en $0 \in \mathbb{C}^{n}$ est dit bien préparé s'il est de la forme $X=T+x^{U} V+x^{U} \mathfrak{R}$, où

- $T$ champ de vecteurs linéaire diagonal 1-résonnant, $x^{U}$ générateur de résonances, $U:=\left(U_{1}, \ldots, U_{n}\right)$ avec $U_{i} \neq 0$,

- $V$ champ de vecteurs linéaire diagonal avec $\mathcal{L}_{V}\left(x^{U}\right) \neq 0$,

- $\mathcal{L}_{\mathfrak{R}}\left(x^{U}\right)=0$ et $\mathfrak{R}:=\sum_{i=1}^{n} f_{i} \partial_{i}, f_{i}=x_{i} \tilde{f}_{i}, \tilde{f}_{i}$ holomorphe en $0 \in \mathbb{C}^{n}$.

Soit $\alpha \in \mathbb{C}^{n}$ tel que $\beta:=(U, \alpha) \neq 0$, et désignons par $\mathcal{E}_{\mu, \alpha}$ l'ensemble des champs de vecteurs bien préparés de la forme

$$
\sum_{i=1}^{n}\left(x_{i}\left(\mu_{i}+\alpha_{i} x^{U}\right)+x^{U} f_{i}(x)\right) \partial_{i},
$$

On note $X_{\mu, \alpha}=\sum_{i=1}^{n} x_{i}\left(\mu_{i}+\alpha_{i} x^{U}\right) \partial_{i}$ leurs forme normale (cf. la proposition 7.2). On suppose

- $\left(H_{1}^{\prime}\right)$ les valeurs propres $\mu_{i}$ toutes situées sur une droite $(d) \subset \mathbb{C}$ passant par l'origine. 
- $\left(H_{2}^{\prime}\right)$ il existe un indice $i_{0}$ tel que $\lambda_{i_{0}} \neq 0$ et tel que

$$
\min _{i \neq i_{0}} \operatorname{Re}\left(\frac{\alpha_{i}}{\beta}-\frac{\mu_{i}}{\mu_{i_{0}}} \frac{\alpha_{i_{0}}}{\beta}\right)>0 .
$$

Soit $\delta$ un nombre complexe de module 1 tel que les nombres $\mu_{i} /(\beta \delta)$ soient tous réels.

ThÉORÈme 8.1 (Stolovitch). - Soient $0<\varepsilon<\frac{\pi}{2}$ fixé et $X \in \mathcal{E}_{\mu, \alpha}$. Sous $\left(H_{1}^{\prime}\right),\left(H_{2}^{\prime}\right)$,

(i) il existe des transformations $\Phi_{j}, j=0,1$, tangentes à l'identité, holomorphes sur $D S_{j}^{U}\left(r, r^{\prime}, \delta\right)$ avec $r, r^{\prime}$ suffisamment petits telles que

$$
\Phi_{j}^{*}(X)=X_{\mu, \alpha} .
$$

(ii) Les transformations $\Phi_{j}$ admettent $\hat{\Phi}$ comme développement asymptotique au sens de Gérard-Sibuya en $x^{U}$ dans $D S_{j}^{U}\left(r, r^{\prime}, \delta\right)$ où $\hat{\Phi}$ est l'unique transformation formelle conjuguant $X \grave{a} X_{\mu, \alpha}$ et préservant $x^{R}$, c.a.d. telle que $x^{R} \circ \hat{\Phi}=x^{U}$.

(iii) Les transformations $\Phi_{j} j=0,1$ préservent $x^{U}$.

(iv) Pour tout $\Lambda \equiv\left(\Lambda_{1}, \ldots, \Lambda_{n}\right) \in \mathbb{C}^{n}$ tel que $\mathcal{L}_{\mathfrak{R}}\left(x^{\Lambda}\right)=0$ on a $x^{\Lambda} \circ \Phi_{j}=x^{\Lambda}$ (quel que soit la détermination de $x^{\Lambda}$ choisie).

REMARQUe. - Le quatrième point du précédent résultat ne figure pas dans l'énoncé original (théorème 3.3.1 dans [16]) mais suit immédiatement de sa démonstration : le système (3.5) dans la preuve du théorème 3.3 .3 de [16] satisfait condition $(r)$ du théorème 2.2.1 de [16], avec $R=\left(\Lambda_{1}, \ldots, \Lambda_{n}\right)$, assurant $x^{\Lambda} \circ \Phi_{j}=x^{\Lambda}$ (le fait que $\Lambda_{i} \notin \mathbb{N}$ n'a aucune incidence).

Application. - On revient à la structure de Poisson $\Pi$ du théorème 3.1. Par la proposition 6.1 on peut supposer $\Pi$ prénormalisé, c'est-à-dire de la forme

$$
\Pi=\Pi^{2}+x^{R} \Pi^{R}+x^{R} \sum_{I \in \mathbb{N}^{n}} x^{I} \Pi^{I},
$$

où les multiindices $I$ apparaissant effectivement dans la somme n'ont pas de composante strictement négative. On rappelle qu'on désigne par $\Pi^{I}$ des champs de bivecteurs quadratiques diagonaux.

Lemme 8.1. - Sous les hypothèses du théorème 3.1 il existe $\mathfrak{B} \subset \mathbb{C}^{n}$ intersection d'un ouvert et d'un sous-ensemble dense de mesure pleine de $\mathbb{C}^{n}$ tel que pour $\Lambda \in \mathfrak{B}$ le champ de vecteurs associé à $\Pi v_{\Lambda} \frac{1}{x^{\Lambda}} X_{\Pi}\left(x^{\Lambda}\right)$, où $v_{\Lambda}$ désigne une unité analytique appropriée, satisfait les hypothèses du théorème 8.1 et de la proposition 7.2. L'ensemble $\mathfrak{B}$ ne dépend que de la partie quadratique $\Pi^{2}$ et de $x^{R} \Pi^{R}$. 
Preuve du lemme 8.1. - Il suffit de montrer que pour $\Lambda$ appartenant à un ensemble dense $\mathfrak{B}^{\prime}$ de mesure pleine de $\mathbb{C}^{n}$ il existe une unité analytique $v_{\Lambda}$ telle que $v_{\Lambda} \frac{1}{x^{\Lambda}} X_{\Pi}\left(x^{\Lambda}\right)$ soit bien préparé et satisfait les hypothèses de la proposition 7.2. Les conditions $\left(H_{1}^{\prime}\right)$ et $\left(H_{2}^{\prime}\right)$ sont alors satisfaites pour $\Lambda \in \mathfrak{B}=\mathfrak{B}^{\prime} \cap \mathfrak{U}$, où $\mathfrak{U}$ désigne l'ouvert où $\left(H_{1}^{\prime}\right)$ et $\left(H_{2}^{\prime}\right)$ sont satisfaites.

Rappelons qu'on note $\Pi^{2}:=\frac{1}{2} \sum_{i \neq j} a_{i j} Y_{i} \wedge Y_{j}$, où $a_{i j}=-a_{j i}$, et $\Pi^{R}:=S \wedge C$, avec $S \in \mathbb{S}$ et $C$ tel que $\mathcal{L}_{C}\left(x^{R}\right) \neq 0$. Posons $\Pi^{R}:=\frac{1}{2} \sum_{i \neq j} b_{i j} Y_{i} \wedge Y_{j}$, où $b_{i j}=-b_{j i}$.

D'après la proposition 7.1, la condition que $\frac{1}{x^{\Lambda}} X_{\Pi^{2}}\left(x^{\Lambda}\right) \in \mathbb{S}$ soit un élément de Cartan de $\mathbb{S}$ (cf. définition 7.1) tel que $\Lambda . S \neq 0$ est satisfaite pour $\Lambda$ appartenant à un ensemble dense de mesure pleine $\mathfrak{B}^{\prime}$ de $\mathbb{C}^{n}$. Considérons $\Lambda:=\left(\Lambda_{1}, \ldots, \Lambda_{n}\right) \in \mathfrak{B}^{\prime}$ et montrons qu'il existe une unité analytique $u_{\Lambda}$ tel que $v_{\Lambda} \frac{1}{x^{\Lambda}} X_{\Pi}\left(x^{\Lambda}\right)$ soit bien préparé, i.e. appartienne à $\mathcal{E}_{\mu, \alpha}$ où

$\mu=\left(\left(\sum_{i=1}^{n} \Lambda_{i} a_{i 1} Y_{1}\right), \ldots,\left(\sum_{i=1}^{n} \Lambda_{i} a_{i n} Y_{n}\right)\right)$ et $\alpha=\left(\left(\sum_{i=1}^{n} \Lambda_{i} b_{i 1} Y_{1}\right), \ldots,\left(\sum_{i=1}^{n} \Lambda_{i} b_{i n} Y_{n}\right)\right)$.

Le champ de vecteurs $\frac{1}{x^{\Lambda}} X_{x^{R} \Pi^{R}}\left(x^{\Lambda}\right)\left(=x^{R} \frac{1}{x^{\Lambda}}\left(\mathcal{L}_{S}\left(x^{\Lambda}\right) C-\mathcal{L}_{C}\left(x^{\Lambda}\right) S\right)\right.$ n'admet pas $x^{R}$ comme intégrale première formelle puisque par hypothèse sur $S, C$ et $\Lambda$ on a $\Lambda . S:=\frac{1}{x^{\Lambda}} X_{S}\left(x^{\Lambda}\right) \neq 0, \mathcal{L}_{C}\left(x^{R}\right) \neq 0$ et $\mathcal{L}_{S}\left(x^{R}\right)=0$. Comme $\frac{1}{x^{\Lambda}} X_{\Pi^{2}}\left(x^{\Lambda}\right) \in$ $\mathbb{S}$ on en déduit $\beta \neq 0$ tel que

$$
\mathcal{L}_{\left[\frac{1}{x^{\Lambda}} X_{\Pi^{2}}\left(x^{\Lambda}\right)+\frac{1}{x^{\Lambda}} X_{\Pi^{R}}\left(x^{\Lambda}\right)\right]}\left(x^{R}\right)=\mathcal{L}_{\left[\frac{1}{x^{\Lambda}} X_{\Pi^{R}}\left(x^{\Lambda}\right)\right]}\left(x^{R}\right)=\beta\left(x^{R}\right)^{2} .
$$

Il suit de (8.2) et de (8.3) qu'il existe des fonctions $g_{i}$ holomorphes sur $\mathbb{C}^{n}$ telles que

$$
\left.\frac{1}{x^{\Lambda}} X_{\Pi}\left(x^{\Lambda}\right)=\sum_{i=1}^{n}\left(x_{i}\left(\mu_{i}+x^{R} \alpha_{i}\right)\right)+x^{R} x_{i} g_{i}(x)\right) \partial_{i}
$$

et

$$
\mathcal{L}_{\frac{1}{x^{\Lambda}} X_{\Pi}\left(x^{\Lambda}\right)}\left(x^{R}\right)=\beta\left(x^{R}\right)^{2}+\left(x^{R}\right)^{2} \sum_{i=1}^{n} g_{i}(x) .
$$

Posons $v_{\Lambda}(x):=\left(1+\frac{1}{\beta} \sum_{i=1}^{n} g_{i}(x)\right)^{-1}$ et $\tilde{X}:=v_{\Lambda} \frac{1}{x^{\Lambda}} X_{\Pi}\left(x^{\Lambda}\right)$. Remarquons que $\tilde{X}$ s'écrit sous la forme

$$
\tilde{X}=\sum_{i=1}^{n}\left(x_{i}\left(\mu_{i}+x^{R} \alpha_{i}\right)+x^{R} x_{i} \tilde{g}_{i}(x)\right) \partial_{i} .
$$

Or, il suit de la définition de $\tilde{X}$ que la dérivée de Lie de $x^{R}$ le long de $\tilde{X}$ vaut $\mathcal{L}_{\tilde{X}}\left(x^{R}\right)=\beta\left(x^{R}\right)^{2}$. Avec

$$
\mathcal{L}_{\left[\sum_{i=1}^{n} x_{i}\left(\mu_{i}+x^{R} \alpha_{i}\right) \partial_{i}\right]}\left(x^{R}\right):=\mathcal{L}_{\left[\frac{1}{x^{\Lambda}} X_{\Pi^{2}}\left(x^{\Lambda}\right)+\frac{1}{x^{\Lambda}} X_{\Pi^{R}}\left(x^{\Lambda}\right)\right]}\left(x^{R}\right)=\beta\left(x^{R}\right)^{2}
$$

BULLETIN DE LA SOCIÉTÉ MATHÉMATIQUE DE FRANCE 
il suit finalement

$$
\mathcal{L}_{\left[x^{R} \sum_{i=1}^{n} x_{i} \tilde{g}_{i} \partial_{i}\right]}\left(x^{R}\right)=0
$$

c'est-à-dire $\tilde{X} \in \mathcal{E}_{\mu, \alpha}$.

Lemme 8.2. - Soit $\mathcal{P}$ une structure de Poisson qui satisfait les hypothèses $\left(P_{1}\right)-\left(P_{4}\right)$ du théorème 3.1, et $f: \mathbb{C}^{n} \rightarrow \mathbb{C}$ une fonction analytique. Alors $f \mathcal{P}$ est également une structure de Poisson, i.e. on a la relation $[f \mathcal{P}, f \mathcal{P}]=0$.

Preuve du lemme 8.2. - Par la proposition 4.1 il existe une transformation formelle $\hat{\Psi}$ telle que $(\hat{\Psi})^{*}(\mathcal{P})=\Pi^{2}+x^{R} \Pi^{R}$. Or $\Pi^{2}+x^{R} \Pi^{R}$ s'écrit sous la forme $S \wedge\left(S^{\prime}+x^{R} C\right)$, avec $S, S^{\prime} \in \mathbb{S}$. On en déduit que $\mathcal{P}$ et donc $f \mathcal{P}$ sont produits extérieurs de deux champs de vecteurs formels. Il suit que le champ de bivecteurs $f \mathcal{P}$ satisfait la relation $[f \mathcal{P}, f \mathcal{P}]=0$.

DÉfinition 8.2. - On dit que $I \in \mathbb{N}^{n}$ est un bon multiindice si $x^{R}$ ne divise pas $x^{I}$. On note $\mathbb{D}$ l'ensemble des bons multi-indices.

Proposition 8.1. - Soient $\mathfrak{B}$ tel que dans le lemme 8.1, $\Lambda \in \mathfrak{B}$ et $v_{\Lambda}$ l'unité analytique fourni par le lemme 8.1. Soit $\Phi_{j}^{\Lambda}$ la transformation fourni par le théorème 8.1 normalisant holomorphiquement le champ de vecteurs $v_{\Lambda} \frac{1}{x^{\Lambda}} X_{\Pi}\left(x^{\Lambda}\right)$ sur le domaine sectoriel $D S_{j}^{R}\left(r, r^{\prime}, \delta\right), j=0,1$. Alors

(i) $\Phi_{j}^{\Lambda}$ préserve $x^{\Lambda}$, c'est-à-dire on a $x^{\Lambda} \circ \Phi_{j}=x^{\Lambda}$;

(ii) $\left(\Phi_{j}^{\Lambda}\right)^{*}\left(v_{\Lambda} \Pi\right)$ s'écrit $\Pi^{2}+x^{R} \Pi^{R}+\mathcal{R}^{\Lambda}$ où $\mathcal{R}^{\Lambda}$ désigne un champ de bivecteurs infiniment plat défini sur $D S_{j}^{R}\left(r, r^{\prime}, \delta\right), j=0,1$;

(iii) $\Phi_{j}^{*}\left(v_{\Lambda} \Pi\right)$ est une structure de Poisson;

(iv) $\mathcal{R}^{\Lambda} s^{\prime}$ écrit

$$
\sum_{I \in \mathbb{D}} x^{I} \frac{1}{2} \sum_{i \neq j} f_{i j}^{\Lambda, I}\left(x^{R}\right) Y_{i} \wedge Y_{j}
$$

ò̀ $f_{i j}^{\Lambda, I}=-f_{j i}^{\Lambda, I}$. Les $f_{i j}^{\Lambda, I}$ sont des fonctions infiniment plates définies sur $U_{i}(r, \delta), j=0,1$, ò̀

$$
U_{i}(r, \delta):=\left\{z \in \mathbb{C}|| z|<r,| \arg z-\left(\arg \delta+\pi\left(j+\frac{1}{2}\right)\right) \mid<\pi-\varepsilon\right\} .
$$

Preuve de la proposition 8.1. - $\Phi_{j}^{\Lambda}$ conjugue $v_{\Lambda} \frac{1}{x^{\Lambda}} X_{\Pi}\left(x^{\Lambda}\right)$ à la forme normale $\frac{1}{x^{\Lambda}} X_{\Pi^{2}}\left(x^{\Lambda}\right)+\frac{1}{x^{\Lambda}} X_{\Pi^{R}}\left(x^{\Lambda}\right)$ sur $D S_{j}^{R}\left(r, r^{\prime}, \delta\right), j=0,1$. Avec $\mathcal{L}_{\frac{1}{x^{\Lambda}} X_{\Pi}\left(x^{\Lambda}\right)}\left(x^{R}\right)=0$ le premier point de la proposition 8.1 suit du théorème 8.1 (iv) et le troisième point suit le lemme 8.2. Le deuxième point de la proposition 8.1 suit de la conjugaison $\left(\Phi_{j}^{\Lambda}\right)^{*}\left(v_{\Lambda} \frac{1}{x^{\Lambda}} X_{\Pi}\left(x^{\Lambda}\right)\right)=\frac{1}{x^{\Lambda}} X_{\Pi^{2}+x^{R} \Pi^{R}}\left(x^{\Lambda}\right)$ et de la proposition 7.2 appliquée à la structure de Poisson $v_{\Lambda} \Pi$ (cf. les lemmes 8.1, 8.2). 
Reste à montrer le quatrième point. D'après le théorème 8.1, les transformation sectorielles $\Phi_{j}^{\Lambda}, j=0,1$ admettent $\hat{\Phi}_{j}^{\Lambda}$ comme développement asymptotique au sens de Gérard-Sibuya en le monôme $y^{R}$ sur $D S_{j}^{R}\left(r, r^{\prime}, \delta\right)$ (cf. section $2)$ : posons $\Phi_{j}^{\Lambda}\left(y_{1}, \ldots, y_{n}\right)=\left(\varphi_{1}(y), \ldots, \varphi_{n}(y)\right)$. Chaque composante $\varphi_{i}$ admet une écriture de la forme

$$
\varphi_{i}(z, y)=\sum_{Q \in \mathbb{N}^{n}} \varphi_{i, Q}\left(y^{R}\right) y^{Q},
$$

tel que $\hat{\varphi}_{i}=\sum_{Q \in \mathbb{N}^{n}} \hat{\varphi}_{i, Q}\left(y^{R}\right) y^{Q}$, et tel que chacune des fonctions $\varphi_{i, j, Q}(z)$ admet $\hat{\varphi}_{i, Q}(z)$ comme développement asymptotique au sens de Poincaré dans le secteur $U_{j}, j=0,1$. D'où

$$
\mathcal{R}^{\Lambda}=\sum_{I \in \mathbb{N}^{n}} x^{I} \frac{1}{2} \sum_{i \neq j} g_{i j}^{\Lambda, I}\left(x^{R}\right) Y_{i} \wedge Y_{j},
$$

où $g_{i j}^{\Lambda, I}=-g_{j i}^{\Lambda, I}$. Les fonctions $g_{i j}^{\Lambda, I}$ sont plates définies sur $U_{j}, j=0,1$. On obtient $\mathcal{R}^{\Lambda}$ sous la forme de la proposition 8.1 (iv) en posant, pour $I \in \mathbb{D}$,

$$
f_{j i}^{\Lambda, I}\left(x^{R}\right):=\sum_{\substack{J=I+l R \\ l \in \mathbb{N}}}\left(x^{R}\right)^{l} g_{j i}^{\Lambda, J}\left(x^{R}\right) .
$$

Nullité du reste infiniment plat. - Soit $\mathcal{R}^{\Lambda}$ tel que dans la proposition 8.1. Posons

$$
\mathcal{R}^{\Lambda}=\sum_{I \in \mathbb{D}} x^{I} \Pi^{I, \Lambda}
$$

Proposition 8.2. - Soient $\mathfrak{B}$ tel que dans le lemme 8.1 et $\mathcal{R}^{\Lambda}$ tel que dans la proposition 8.1. Il existe $\mathcal{C} \subset \mathcal{B}$ de mesure de Lebesque nulle tel que pour $\Lambda \in \mathcal{B}-\mathcal{C}$

$$
\mathcal{R} \neq 0 \Rightarrow\left[\Pi^{2}, x^{I_{0}} \Pi^{I_{0}, \Lambda}\right]=0,
$$

où $\Pi^{I_{0}, \Lambda}$ désigne un terme de plus petit degré non-nul de (8.4). L'ensemble $\mathfrak{C}$ ne dépend que de la partie quadratique $\Pi^{2}$ et de $x^{R} \Pi^{R}$.

Corollaire 8.1. - Soient $\mathcal{C}$ et $x^{I_{0}} \Pi^{I_{0}, \Lambda}\left(x^{R}\right)$ telles que dans la proposition 8.2. Pour $\Lambda \in \mathcal{B}-\mathcal{C}$

$$
x^{I_{0}} \Pi^{I_{0}, \Lambda}\left(x^{R}\right) \neq 0 \Rightarrow \frac{1}{x^{\Lambda}} X_{\left[x^{I} \Pi^{i_{0}, \Lambda}\left(x^{R}\right)\right]}\left(x^{\Lambda}\right) \neq 0 .
$$

Preuve du théorème 3.1. - Montrons que pour $\Lambda \in \mathcal{B}-\mathcal{C}$ tel que dans la proposition $8.2, v_{\Lambda}$ tel que dans le lemme 8.1 et $\Phi_{j}^{\Lambda}, j=0,1$ tel que dans la proposition 8.1 , on a la relation de conjugaison

$$
\left(\Phi_{j}^{\Lambda}\right)^{*}\left(v_{\Lambda} \Pi\right)=\Pi^{2}+x^{R} \Pi^{R}
$$


sur $D S_{j}^{R}\left(r, r^{\prime}, \delta\right), j=0,1$. Ceci revient à montrer que $\mathcal{R}^{\Lambda}$ tel que dans la proposition 8.1 est nul c'est-à-dire à montrer que $x^{I_{0}} \Pi^{I_{0}, \Lambda}\left(x^{R}\right)$ tel que dans la proposition 8.2 est nul.

D'après le corollaire 8.1, $x^{I_{0}} \Pi^{I_{0}, \Lambda}\left(x^{R}\right) \neq 0$ implique $\frac{1}{x^{\Lambda}} X_{\left[x^{I_{0}} \Pi^{I_{0}, \Lambda}\left(x^{R}\right)\right]}{ }^{\left(x^{\Lambda}\right) \neq}$ 0 . Si $x^{I_{0}} \Pi^{I_{0}, \Lambda}\left(x^{R}\right)$ est non nul on a une contradiction avec le fait que $\Phi_{j}^{\Lambda}$ conjugue le champ de vecteurs $\frac{1}{x^{\Lambda}} X_{v_{\Lambda} \Pi}\left(x^{\Lambda}\right)$ à la forme normale polynomiale $\frac{1}{x^{\Lambda}} X_{\Pi^{2}}\left(x^{\Lambda}\right)+\frac{1}{x^{\Lambda}} X_{\Pi^{R}}\left(x^{\Lambda}\right)$ sur $D S_{j}^{R}\left(r, r^{\prime}, \delta\right), j=0,1$. Les autres affirmations du théorème 3.1 suivent du théorème 8.1 , du lemme 8.1 et des propositions 8.2 et 8.1 .

Preuve du corollaire 8.1. - Soit $p=\left(p_{1}, \ldots, p_{n}\right)$ un point dans le domaine sectoriel $D S_{j}^{R}\left(r, r^{\prime}, \delta\right) j=0,1$ dont toutes les coordonnées soient non nulles, c'est-à-dire $p_{1} \neq 0, \ldots, p_{n} \neq 0$. Par la proposition $8.2, \operatorname{sur} D S_{j}^{R}\left(r, r^{\prime}, \delta\right), j=$ 0,1 , on a

$$
\begin{aligned}
& {\left[\Pi^{2}, x^{I_{0}} \Pi^{I_{0}, \Lambda}\left(x^{R}\right)\right] } \\
= & {\left[\Pi^{2}, x^{I_{0}} \frac{1}{2} \sum_{i \neq j} f_{i j}^{I_{0}, \Lambda}\left(x^{R}\right) Y_{i} \wedge Y_{j}\right] } \\
= & 0 .
\end{aligned}
$$

Soient $a_{i j}^{p, I_{0}} \in \mathbb{C}, 1 \leq i, j \leq n$ tels que $a_{i j}^{p, I_{0}}:=f_{i j}^{I_{0}, \Lambda}\left(p^{R}\right)$. En utilisant les relations (2.10), (2.6) on déduit de $S \in \mathbb{S} \Rightarrow \mathcal{L}_{S}\left(x^{R}\right)=0$ que pour $x=p$ on a

$$
\left[\Pi^{2}, x^{I_{0}} \frac{1}{2} \sum_{i \neq j} f_{i j}^{I_{0}, \Lambda}\left(x^{R}\right) Y_{i} \wedge Y_{j}\right]=\left[\Pi^{2}, x^{I_{0}} \frac{1}{2} \sum_{i \neq j} a_{i j}^{p, I_{0}} Y_{i} \wedge Y_{j}\right]=0 .
$$

Or, d'après le lemme 2.1, le terme

$$
\left[\Pi^{2}, x^{I_{0}} \frac{1}{2} \sum_{i \neq j} a_{i j}^{p, I_{0}} Y_{i} \wedge Y_{j}\right]
$$

s'écrit sous la forme $x^{I_{0}} \sum_{i<j<k} a_{i j k} Y_{i} \wedge Y_{j} \wedge Y_{k}, a_{i j k} \in \mathbb{C}$. Comme toutes les coordonnées de $p$ sont non nulles, le fait que (8.6) s'annule en $p$, implique que pour tout $x \in D S_{j}^{R}\left(r, r^{\prime}, \delta\right)$ on a

$$
\left[\Pi^{2}, x^{I_{0}} \frac{1}{2} \sum_{i \neq j} a_{i, j}^{p, I_{0}} Y_{i} \wedge Y_{j}\right]=0
$$

Posons $\Pi^{p, I_{0}}:=\frac{1}{2} \sum_{i \neq j} a_{i, j}^{p, I_{0}} Y_{i} \wedge Y_{j}$. On a donc

$$
\left[\Pi^{2}, x^{I_{0}} \Pi^{p, I_{0}}\right]=0 .
$$

TOME $137-2009-\mathrm{N}^{\circ} 3$ 
Or, d'après (2.6) on a en $x=p$

$$
\frac{1}{x^{\Lambda}} X_{x^{I_{0}} \Pi^{p, I_{0}}}\left(x^{\Lambda}\right)=\frac{1}{x^{\Lambda}} X_{x^{I_{0} \Pi^{I_{0}, \Lambda}\left(x^{R}\right)}}\left(x^{\Lambda}\right) .
$$

D'après le lemme 7.1 (i), si $x^{I} \Pi^{p, I_{0}} \neq 0$, il suit de (8.7) que

$$
\frac{1}{x^{\Lambda}} X_{x^{I_{0}} \Pi^{p, I_{0}}}\left(x^{\Lambda}\right) \neq 0
$$

et le corollaire 8.1 suit de (8.8).

\section{Preuve de la proposition 8.2}

Cette section est consacrée à la preuve de la proposition 8.2. Dans toute la section on suppose les hypothèses de la proposition 8.2 satisfaites ainsi que $\Lambda \in \mathfrak{B}$ (cf. le lemme 8.1), $v_{\Lambda}, \Phi_{j}^{\Lambda}$ et $\mathcal{R}^{\Lambda}$ telles que dans la proposition 8.1.

On commence par établir des résultats préliminaires obtenus à partir des identités de Jacobi $\left\{x^{\Lambda},\left\{x_{i}, x_{j}\right\}\right\}+\left\{x_{i},\left\{x_{j}, x^{\Lambda}\right\}\right\}+\left\{x_{j}\left\{x^{\Lambda}, x_{i}\right\}\right\}=0$, où ici le crochet désigne le crochet de Poisson associé à $\left(\Phi_{j}^{\Lambda}\right)^{*}\left(v_{\Lambda} \Pi\right), j=0,1$.

Notations (en outre des notations de la section précédente). Soient $V$ un champ de vecteurs linéaire diagonal et $I \in \mathbb{N}^{n}$. Désignons par $V . I$ le nombre complexe $\frac{1}{x^{I}} \mathcal{L}_{V}\left(x^{I}\right)$ et notons $B_{\Lambda}:=\frac{1}{x^{\Lambda}} X_{\Pi^{R}}\left(x^{\Lambda}\right)$ et $A_{\Lambda}:=\frac{1}{x^{\Lambda}} X_{\Pi^{2}}\left(x^{\Lambda}\right)$. On pose $N:=\Pi^{2}+x^{R} \Pi^{R}$ ainsi que

$\Pi^{R}:=\frac{1}{2} \sum_{i \neq j} b_{i j} Y_{i} \wedge Y_{j}, \quad N:=\frac{1}{2} \sum_{i \neq j} n_{i j}(x) Y_{i} \wedge Y_{j}, \quad \mathcal{R}^{\Lambda}(x):=\frac{1}{2} \sum_{i \neq j} r_{i j}^{\Lambda}(x) Y_{i} \wedge Y_{j}$. où $b_{i j}=-b_{j i}, n_{i j}=-n_{j i}$ et $r_{i j}^{\Lambda}=-r_{j i}^{\Lambda}$.

Donc $\left(\Phi_{j}^{\Lambda}\right)^{*}\left(v_{\Lambda} \Pi\right)=N+\mathcal{R}^{\Lambda}$, et on rappelle $X_{R^{\Lambda}}\left(x^{\Lambda}\right)=0$ ainsi que $\mathcal{R}^{\Lambda}=$ $\sum_{I \in \mathbb{D}} x^{I} f_{i j}^{I, \Lambda}\left(x^{R}\right) Y_{i} \wedge Y_{j}$ (cf. définition 8.2). Considérons l'identité de Jacobi

$$
\left\{x^{\Lambda},\left\{x_{i_{0}}, x_{j_{0}}\right\}\right\}+\left\{x_{i_{0}},\left\{x_{j_{0}}, x^{\Lambda}\right\}\right\}+\left\{x_{j_{0}}\left\{x^{\Lambda}, x_{i_{0}}\right\}\right\}=0 .
$$

Le fait que la forme normale $N$ est une structure de Poisson implique que la somme des termes de (9.1) qui ne font pas intervenir $\mathcal{R}^{\Lambda}$, c'est-à-dire des fonctions $r_{i j}^{\Lambda}$ est nulle.

On en déduit que la somme des termes de (9.1) qui comportent au moins un terme en un $r_{i j}^{\Lambda}$ est nulle. Comme $X_{\mathcal{R}^{\Lambda}}\left(x^{\Lambda}\right)=0$, cette relation s'écrit

$$
\mathcal{L}_{X_{N}\left(x^{\Lambda}\right)}\left(\left\{x_{i_{0}}, x_{j_{0}}\right\}_{\mathcal{R}^{\Lambda}}\right)+\mathcal{L}_{X_{\mathcal{R}^{\Lambda}}\left(x_{j_{0}}\right)}\left(\left\{x^{\Lambda}, x_{i_{0}}\right\}_{N}\right)+\mathcal{L}_{X_{\mathcal{R}^{\Lambda}}\left(x_{i_{0}}\right)}\left(\left\{x_{j_{0}}, x^{\Lambda}\right\}_{N}\right)=0 .
$$

Lemme 9.1. - (i) Pour tout $1 \leq i_{0}, j_{0} \leq n$ on a la relation

$$
\mathcal{L}_{\left[\frac{1}{x_{0}} X_{\mathcal{R}^{\Lambda}}\left(x_{i_{0}}\right)\right]}\left(\left(-n_{j_{0}}^{\Lambda}\right)\right)+\mathcal{L}_{\left[\frac{1}{x_{j_{0}}} X_{\mathcal{R}^{\Lambda}}\left(x_{j_{0}}\right)\right]}\left(n_{i_{0}}^{\Lambda}\right)+\mathcal{L}_{\left[\frac{1}{x^{\Lambda}} X_{N}\left(x^{\Lambda}\right)\right]}\left(r_{i_{0} j_{0}}^{\Lambda}\right)=0 .
$$


(ii) Soit $I \in \mathbb{D}$ fixé. Alors les fonctions $f_{i j}^{I, \Lambda}$ sont solutions du système à singularité irrégulière suivant, chaque équation étant indexée par un couple $\left(i_{0}, j_{0}\right)$ avec $i_{0} \neq j_{0}:$

$$
\begin{gathered}
b_{i_{0}}^{\Lambda}\left(\sum_{i=1}^{n} f_{j_{0} i}^{I, \Lambda} \cdot R_{i}\right) u-b_{j_{0}}^{\Lambda}\left(\sum_{j=1}^{n} f_{i_{0} j}^{I, \Lambda} R_{j}\right) u+\left(A_{\Lambda} . I+u B_{\Lambda} \cdot I\right) \tilde{f}_{i_{0} j_{0}}^{I, \Lambda}(u) \\
(9.4)+(u)^{2}(S+C) . \Lambda \partial_{u}\left(\tilde{f}_{i_{0} j_{0}}^{I, \Lambda}\right)(u)=0
\end{gathered}
$$

ò̀ $b_{j}^{\Lambda}:=\sum_{i=1}^{n} b_{i j} \lambda_{i}, R:=\left(R_{1}, \ldots, R_{n}\right)$.

Preuve du lemme 9.1. - Fixons $\Lambda \in \mathfrak{B}$ (cf. le lemme 8.1). Dans cette preuve on omet d'indexer systématiquement par $\Lambda$. D'après (9.2) on a

$$
\begin{aligned}
\left\{x_{i_{0}}, x_{j_{0}}\right\}_{\mathcal{R}} & =\mathcal{L}_{X_{\mathcal{R}}\left(x_{i_{0}}\right)}\left(x_{j_{0}}\right) \\
& =\mathcal{L}_{\left(x_{i_{0}} \sum_{j=1}^{n} r_{i_{0} j} Y_{j}\right)}\left(x_{j_{0}}\right) \\
& =\left(r_{i_{0} j_{0}} x_{i_{0}} x_{j_{0}}\right) .
\end{aligned}
$$

On a donc

$$
\begin{aligned}
\mathcal{L}_{X_{N}\left(x^{\Lambda}\right)}\left(\left\{x_{i_{0}}, x_{j_{0}}\right\}_{\mathcal{R}}\right)= & \left(x_{i_{0}} x_{j_{0}} \mathcal{L}_{X_{N}\left(x^{\Lambda}\right)}\left(r_{i_{0} j_{0}}\right)\right)+\left(r_{i_{0} j_{0}} x_{j_{0}} \mathcal{L}_{X_{N}\left(x^{\Lambda}\right)}\left(x_{i_{0}}\right)\right) \\
& +\left(r_{i_{0} j_{0}} x_{i_{0}} \mathcal{L}_{X_{N}\left(x^{\Lambda}\right)}\left(x_{j_{0}}\right)\right) \\
= & \left(\mathcal{L}_{\frac{1}{x^{\Lambda}} X_{N}\left(x^{\Lambda}\right)}\left(r_{i_{0}, j_{0}}\right)\right)\left[x^{\Lambda} x_{i_{0}} x_{j_{0}}\right]+\left(r_{i_{0} j_{0}} n_{i_{0}}^{\Lambda}\right)\left[x^{\Lambda} x_{i_{0}} x_{j_{0}}\right] \\
& +\left(r_{i_{0} j_{0}} n_{j_{0}}^{\Lambda}\right)\left[x^{\Lambda} x_{i_{0}} x_{j_{0}}\right],
\end{aligned}
$$

où on note $n_{i_{0}}^{\Lambda}\left(\operatorname{resp} n_{j_{0}}^{\Lambda}\right)$ la fonction $\sum_{j=1}^{n} n_{j i_{0}} \lambda_{j}\left(\operatorname{resp} . \sum_{i=1}^{n} n_{i j_{0}} \lambda_{i}\right)$.

De même, en utilisant $X_{\mathcal{R}}\left(x^{\Lambda}\right)=0$ on obtient,

$\mathcal{L}_{X_{\mathcal{R}}\left(x_{j_{0}}\right)}\left(\left\{x^{\Lambda}, x_{i_{0}}\right\}_{N}\right)=\left(\mathcal{L}_{\frac{1}{x_{j_{0}}} X_{\mathcal{R}}\left(x_{j_{0}}\right)}\left(n_{i_{0}}^{\Lambda}\right)\right)\left[x^{\Lambda} x_{i_{0}} x_{j_{0}}\right]+\left(n_{i_{0}}^{\Lambda} r_{j_{0} i_{0}}\right)\left[x^{\Lambda} x_{i_{0}} x_{j_{0}}\right]$,

$$
\mathcal{L}_{X_{\mathcal{R}}\left(x_{i_{0}}\right)}\left\{x_{j_{0}}, x^{\Lambda}\right\}_{N}=\left(\mathcal{L}_{\frac{1}{x_{i_{0}}} X_{\mathcal{R}}\left(x_{i_{0}}\right)}\left(-n_{j_{0}}^{\Lambda}\right)\right)\left[x^{\Lambda} x_{i_{0}} x_{j_{0}}\right]+\left(-n_{j_{0}}^{\Lambda} r_{i_{0} j_{0}}\right)\left[x^{\Lambda} x_{i_{0}} x_{j_{0}}\right]
$$

Injectons maintenant (9.5), (9.6) et (9.7) dans (9.2). Comme toutes les termes de (9.5), (9.6) et (9.7) comportent $x^{\Lambda} x_{i_{0}} x_{j_{0}}$ en facteur, on peut simplifier par $x^{\Lambda} x_{i_{0}} x_{j_{0}}$. La relation (9.2), c'est-à-dire $(9.5)+(9.6)+(9.7)=0$ devient

$$
\mathcal{L}_{\frac{1}{x_{i_{0}}}} X_{\mathcal{R}\left(x_{i_{0}}\right)}\left(\left(-n_{j_{0}}^{\Lambda}\right)\right)+\mathcal{L}_{\frac{1}{x_{j_{0}}} X_{\mathcal{R}}\left(x_{j_{0}}\right)}\left(n_{i_{0}}^{\Lambda}\right)+\mathcal{L}_{\frac{1}{x^{\Lambda}} X_{N}\left(x^{\Lambda}\right)}\left(r_{i_{0} j_{0}}\right)=0
$$

d'où le lemme 9.1 (i).

Remarquons qu'on a $n_{i j}=a_{i j}+x^{R} b_{i j}$ et $r_{i j}:=\sum_{I \in \mathbb{D}} x^{I} f_{i j}^{I}$. Notons $\mathcal{R}^{I}:=$ $\frac{1}{2} \sum_{i \neq j} x^{I} f_{i j}^{I} Y_{i} \wedge Y_{j}$ la composante en $x^{I}$ de $\mathcal{R}$. 
Développons $\mathcal{L}_{\frac{1}{x^{\Lambda}} X_{N}\left(x^{\Lambda}\right)}\left(x^{I} f_{i_{0} j_{0}}^{I}\right)$ : on a

$$
\mathcal{L}_{\frac{1}{x^{\Lambda}} X_{N}\left(x^{\Lambda}\right)}\left(x^{I} f_{i_{0} j_{0}}^{I}\right)=f_{i_{0} j_{0}}^{I} \mathcal{L}_{\frac{1}{x^{\Lambda}} X_{N}\left(x^{\Lambda}\right)}\left(x^{I}\right)+x^{I} \mathcal{L}_{\frac{1}{x^{\Lambda}} X_{N}\left(x^{\Lambda}\right)}\left(f_{i_{0} j_{0}}^{I}\right) .
$$

On a $\mathcal{L}_{\frac{1}{x^{\Lambda}} X_{N}\left(x^{\Lambda}\right)}\left(f_{i_{0} j_{0}}^{I}\right)(x)=\left.\mathcal{L}_{\frac{1}{x^{\Lambda}} X_{N}\left(x^{\Lambda}\right)}\left(x^{R}\right)\left(\partial_{u} f_{i_{0} j_{0}}^{I}(u)\right)\right|_{u=x^{R}}$. La relation (9.9) devient donc

$$
\mathcal{L}_{\frac{1}{x^{\Lambda}} X_{N}\left(x^{\Lambda}\right)}\left(x^{I} f_{i_{0} j_{0}}^{I}\right)=f_{i_{0} j_{0}}^{I} \mathcal{L}_{\frac{1}{x^{\Lambda}} X_{N}\left(x^{\Lambda}\right)}\left(x^{I}\right)+\left.x^{I} \mathcal{L}_{\frac{1}{x^{\Lambda}} X_{N}\left(x^{\Lambda}\right)}\left(x^{R}\right)\left(\partial_{u} f_{i_{0} j_{0}}^{I}(u)\right)\right|_{u=x^{R}} .
$$

Rappelons l'égalité $\frac{1}{x^{\Lambda}} X_{N} x^{\Lambda}=\frac{1}{x^{\Lambda}} X_{\Pi^{2}}\left(x^{\Lambda}\right)+\frac{x^{R}}{x^{\Lambda}}\left(\mathcal{L}_{S}\left(x^{\Lambda}\right) C-\mathcal{L}_{C}\left(x^{\Lambda}\right) S\right)$. Elle peut se mettre sous la forme

$$
\frac{1}{x^{\Lambda}} X_{N} x^{\Lambda}=\frac{1}{x^{\Lambda}} X_{\Pi^{2}}\left(x^{\Lambda}\right)+x^{R}((S . \Lambda) C-(C . \Lambda) S) .
$$

Comme $S$ et $\frac{1}{x^{\Lambda}} X_{\Pi^{2}}\left(x^{\Lambda}\right)$ appartiennent à $\mathbb{S}, \mathcal{L}_{\frac{1}{x^{\Lambda}} X_{N}\left(x^{\Lambda}\right)}\left(x^{R}\right)=\mathcal{L}_{x^{R}(S . \Lambda) C}\left(x^{R}\right)$. La relation (9.10) devient donc

$$
\begin{aligned}
\mathcal{L}_{\frac{1}{x^{\Lambda}} X_{N}\left(x^{\Lambda}\right)}\left(x^{I} f_{i_{0} j_{0}}^{I}\right) & =f_{i_{0} j_{0}}^{I} \mathcal{L}_{\frac{1}{x^{\Lambda}} X_{N}\left(x^{\Lambda}\right)}\left(x^{I}\right)+\left.x^{I} x^{R}(S . \Lambda) \mathcal{L}_{C}\left(x^{R}\right)\left(\partial_{u} f_{i_{0} j_{0}}^{I}(u)\right)\right|_{u=x^{R}} \\
& =f_{i_{0} j_{0}}^{I} \mathcal{L}_{\frac{1}{x^{\Lambda}} X_{N}\left(x^{\Lambda}\right)}\left(x^{I}\right)+\left.x^{I}\left(x^{R}\right)^{2}(S . \Lambda)(C . \Lambda)\left(\partial_{u} f_{i_{0} j_{0}}^{I}(u)\right)\right|_{u=x^{R}} .
\end{aligned}
$$

Or, $\mathcal{L}_{\frac{1}{x^{\Lambda}} X_{N}\left(x^{\Lambda}\right)}\left(x^{I}\right)=x^{I}\left(A_{\Lambda} \cdot I+x^{R} B_{\Lambda} \cdot I\right)$. La relation $(9.11)$ devient, si on note $I:=\left(I_{1}, \ldots, I_{n}\right)$,

$\mathcal{L}_{\frac{1}{x^{\Lambda}} X_{N}\left(x^{\Lambda}\right)}\left(x^{I} f_{i_{0} j_{0}}^{I}\right)=x^{I}\left[\left(A_{\Lambda} \cdot I+x^{R} B_{\Lambda} \cdot I\right) f_{i_{0} j_{0}}^{I}\left(x^{R}\right)+\left.\left(x^{R}\right)^{2}(S+C) \cdot \Lambda\left(\partial_{u} f_{i_{0} j_{0}}^{I}(u)\right)\right|_{u=x^{R}}\right]$.

Développons $\mathcal{L}_{\frac{1}{x_{i_{0}}} X_{\mathcal{R}^{I}}\left(x_{i_{0}}\right)}\left(\left(-n_{j_{0}}^{\Lambda}\right)\right)$ : posons $n_{j_{0}}^{\Lambda}:=\sum_{i=1}^{n} n_{i j_{0}} \lambda_{i}$.

$$
\begin{aligned}
\mathcal{L}_{\frac{1}{x_{i_{0}}} X_{\mathcal{R}^{I}}\left(x_{i_{0}}\right)}\left(\left(-n_{j_{0}}^{\Lambda}\right)\right) & =\mathcal{L}_{\frac{1}{x_{i_{0}}} X_{\mathcal{R}^{I}}\left(x_{i_{0}}\right)}\left(x^{R}(-1) b_{j_{0}}^{\Lambda}\right) \\
& =-b_{j_{0}}^{\Lambda}\left(\sum_{j=1}^{n} f_{i_{0} j}^{I} R_{j}\right) x^{I} x^{R} .
\end{aligned}
$$

Développons $\mathcal{L}_{\frac{1}{x_{j_{0}}} X_{\mathcal{R}^{I}}\left(x_{j_{0}}\right)}\left(n_{i_{0}}^{\Lambda}\right)$ : on a $\left(-n_{i_{0}}^{\Lambda}\right)=\sum_{j=1}^{n} n_{j i_{0}} \lambda_{j}$, et comme $n_{j i_{0}}=$ $a_{j i_{0}}+x^{R} b_{j i_{0}}$ on définit $b_{i_{0}}^{\Lambda}$ comme la fonction $\sum_{j=1}^{n} b_{j i_{0}} \lambda_{j}$. D'où

$$
\begin{aligned}
\mathcal{L}_{\frac{1}{x_{j_{0}}} X_{\mathcal{R}}\left(x_{j_{0}}\right)}\left(n_{i_{0}}^{\Lambda}\right) & =\mathcal{L}_{\frac{1}{x_{j_{0}}}} X_{\mathcal{R}^{I}\left(x_{j_{0}}\right)}\left(x^{R} b_{i_{0}}^{\Lambda}\right) \\
& =b_{i_{0}}^{\Lambda}\left(\sum_{i=1}^{n} f_{j_{0} i}^{I} R_{i}\right) x^{I} x^{R} .
\end{aligned}
$$

En regardant les composantes en $x^{I}$ de (9.8), on obtient que les $f_{i j}^{I}$ doivent vérifier le système d'équations $(9.12)+(9.13)+(9.14)=0$, qui, simplifiant par $x^{I}$ et en posant $u=x^{R}$ devient le système (9.4). 
Posons $\alpha^{\Lambda}:=-(S+C) . \Lambda$ et $\beta_{I}^{\Lambda}:=A_{\Lambda} . I$ ainsi que $\gamma_{I}^{\Lambda}:=B_{\Lambda} . I$. En notons $F_{I}^{\Lambda}$ le vecteur $\left(\tilde{f}_{i j}^{I, \Lambda}\right)_{i \neq j}$, on peut écrire le système (9.4) sous la forme

$$
u^{2} \alpha^{\Lambda} \partial_{u} F_{I}^{\Lambda}=\beta_{I}^{\Lambda} F^{\Lambda}+u \gamma_{I}^{\Lambda} F^{\Lambda}+u \mathcal{M}^{\Lambda} F^{\Lambda},
$$

où $\mathcal{M}^{\Lambda}$ est une matrice $n(n-1) \times n(n-1)$ à coefficients appartenant à $\mathbb{C}$.

Avant de poursuivre, on a besoin de fixer la terminologie : soit $\mathcal{N}$ une matrice $k \times k$, qu'on identifie à un endomorphisme de $\mathbb{C}^{k} . E_{\mu} \subset \mathbb{C}^{k}$ est un sous-espace caractéristique associé à une valeur propre $\mu$ de $\mathcal{N}$, si $v \in E_{\mu} \Leftrightarrow \exists m ; v \in$ $\operatorname{Ker}(\mathcal{N}-\mu \mathrm{Id})^{m}$.

On sait qu'il existe un changement de coordonnées linéaire de $\mathbb{C}^{k}$ tel que $\mathcal{N}$ s'écrit sous forme de Jordan. Si $\mathcal{N}$ est sous forme de Jordan, c'est-à-dire diagonale par blocs de Jordan de la forme

$$
\left(\begin{array}{ccccc}
\mu_{p} & 1 & 0 & \ldots & 0 \\
0 & \ddots & \ddots & \ddots & \vdots \\
\vdots & \ddots & \ddots & \ddots & 0 \\
\vdots & & \ddots & \ddots & 1 \\
0 & \ldots & \ldots & 0 & \mu_{p}
\end{array}\right) .
$$

Chaque espace caractéristique $E_{\mu}$ est somme directe $E_{\mu}=E_{\mu}^{1} \oplus \cdots \oplus E_{\mu}^{p}$, avec $\mathcal{N}\left(E_{\mu}^{i}\right) \subset E_{\mu}^{i}$ et tel que la restriction de $\mathcal{N}$ à $E_{\mu}^{i}$ est un bloc de Jordan.

Soit maintenant $\widetilde{\mathcal{M}}^{\Lambda}$ une forme de Jordan de $\mathcal{M}^{\Lambda}$. Soit $y_{l, m}=\sum_{i \neq j} c_{i j}^{\Lambda, l m} x_{i j}$ le changement de variable $\mathbb{C}$-linéaire qui transforme $\mathcal{M}^{\Lambda}$ en $\widetilde{\mathcal{M}}^{\Lambda}$. En posant $G_{I}^{\Lambda}:=\left(g_{l m}^{I, \Lambda}\right)$, avec $g_{l m}^{I, \Lambda}:=\sum_{i \neq j} c_{i j}^{\Lambda, l m} \tilde{f}_{i j}^{I, \Lambda}$, le système (9.15) devient

$$
u^{2} \alpha^{\Lambda} \partial_{u} G_{I}^{\Lambda}=\beta_{I}^{\Lambda} G^{\Lambda}+u \gamma_{I}^{\Lambda} G_{I}^{\Lambda}+u \widetilde{\mathcal{M}}^{\Lambda} G_{I}^{\Lambda} .
$$

Soit $\eta^{\Lambda}$ une valeur propre de $\widetilde{\mathcal{M}}^{\Lambda}$. Considérons un sous-système de (9.16) à un sous-espace $E_{\eta^{\Lambda}}^{p}$ de dimension $q$, correspondant à un bloc de Jordan de $\mathcal{M}^{\Lambda}$ appelé $\mathcal{B}_{\eta^{\Lambda}}^{\Lambda, p}$, associé à la valeur propre $\eta^{\Lambda}$ :

$$
u^{2} \alpha^{\Lambda} \partial_{u} \bar{G}_{I}^{\Lambda}=\beta_{I}^{\Lambda} \bar{G}_{I}^{\Lambda}+u \gamma_{I}^{\Lambda} \bar{G}_{I}^{\Lambda}+u \mathcal{B}_{\eta^{\Lambda}}^{\Lambda, p} \bar{G}_{I}^{\Lambda} .
$$

En réindexant (9.17) s'écrit

$$
u^{2} \alpha^{\Lambda} \partial_{u} g_{k}=\left\{\begin{array}{l}
\beta_{I}^{\Lambda} g_{k}+u \gamma_{I}^{\Lambda} g_{k}+u \eta^{\Lambda} g_{k} \text { pour } k=1 \\
\beta_{I}^{\Lambda} g_{k}+u \gamma_{I}^{\Lambda} g_{k}+u \eta^{\Lambda} g_{k}+u g_{k-1} \text { pour } 1<k<q .
\end{array}\right.
$$

On a le lemme suivant : 
LEMME 9.2. - Choisissons sur chaque secteur $U_{j}(r, \delta), j=0,1$, (cf. la proposition 8.1) une détermination du logarithme. Les solution du sous-système (9.18) s'écrivent sous la forme $g_{k}=\sum_{l \in\{0, \ldots, k\}} \tau_{l}^{k}(\ln (u))^{l}(u)^{-\left(\frac{\gamma_{I}^{\Lambda}+\eta^{\Lambda}}{\alpha^{\Lambda}}\right)} \exp \left(\frac{-\beta_{I}^{\Lambda}}{\alpha^{\Lambda} u}\right)$, où les $\tau_{l}^{k}$ sont certaines constantes.

On déduit du lemme 9.2 que les fonctions $f_{i j}^{\Lambda, I}$, solutions du système linéaire (9.15) s'écrivent tous comme une combinaison $\mathbb{C}$-linéaire de fonctions $f^{l, i, I, \Lambda}$ définies par

$$
f^{l, i, I, \Lambda}=(\ln (u))^{l}(u)^{-\left(\frac{\gamma_{I}^{\Lambda}+\eta_{i}^{\Lambda}}{\alpha^{\Lambda}}\right)} \exp \left(\frac{-\beta_{I}^{\Lambda}}{\alpha^{\Lambda} u}\right),
$$

où $l \leq l_{0}$, avec $l_{0}$ la taille maximale d'un bloc de Jordan de $\mathcal{M}^{\Lambda}$, et $\beta_{I}^{\Lambda}:=A_{\Lambda} . I$, $\gamma_{I}^{\Lambda}=B_{\Lambda} . I$, ainsi le fait que $\alpha^{\Lambda}$.

Preuve du lemme 9.2. - On procède par récurrence sur $0 \leq k<q$. Si $k=0$, il est bien connu qu'on a

$$
g_{0}=\tau_{0}^{0} u^{-\left(\frac{1}{\alpha^{\Lambda}}\left(\gamma_{I}^{\Lambda}+\eta^{\Lambda}\right)\right)} e^{-\left(\frac{\beta_{I}^{\Lambda}}{\alpha^{\Lambda} u}\right)},
$$

où $\omega$ est un paramètre traduisant les conditions initiales, le lemme 9.2 est donc vérifié pour la solution de la première équation du système (9.18).

Supposons qu'on a $g_{k}$ pour $k \leq e$ sous la forme donnée par le lemme 9.2, c'est-à-dire

$$
g_{k}=\sum_{l \in\{0, \ldots, k\}} \tau_{l}^{k}(\ln (u))^{l}(u)^{-\left(\frac{\gamma_{I}^{\Lambda}+\eta^{\Lambda}}{\alpha^{\Lambda}}\right)} e^{\left(\frac{-\beta_{I}^{\Lambda}}{\alpha^{\Lambda} u}\right)} .
$$

Il est bien connu que $g_{e+1}$ est somme d'une solution de l'équation homogène associée, c'est-à-dire $g_{0}=\omega u^{-\left(\frac{1}{\alpha^{\Lambda}}\left(\gamma_{I}^{\Lambda}+\eta^{\Lambda}\right)\right)} e^{-\frac{\beta_{I}^{\Lambda}}{\alpha^{\Lambda} u}}$, et d'une solution particulière. Cherchons une solution particulière, par la méthode de la variation de la constante, sous la forme $E(u) g_{0}$ :

En injectant $E(u) g_{0}$ dans la $e+1$-ème équation, dans laquelle on a remplacé $g_{e}$ par

$$
\sum_{l \in\{0, \ldots, e\}} \tau_{l}^{e}\left(\ln ^{l}(u)\right)(u)^{-\frac{\gamma_{I}^{\Lambda}+\eta^{\Lambda}}{\alpha^{\Lambda}}} \exp \left(\frac{-\beta_{I}^{\Lambda}}{\alpha^{\Lambda} u}\right),
$$

on obtient $\alpha^{\Lambda} \partial_{u} E=u^{-1} \sum_{l \in\{0, \ldots, e\}} \tau_{l}^{e} \ln ^{l}(u)$. En intégrant, on en déduit $\alpha^{\Lambda} E=\sum_{l \in\{0, \ldots, e\}} \frac{\tau_{l}^{e}}{l+1} \ln ^{l+1}(u)+C$.

On donc montré le résultat suivant : 
Proposition 9.1. - Soient fixées $I \in \mathbb{D}$, ainsi qu'une détermination du logarithme dans le secteur $U_{j}(r, \delta), j=0,1$ (cf. la proposition 8.1). Alors

(i) Pour tout $i \neq j$ et $\Lambda \in \mathfrak{B}$ fixé, $f_{i j}^{I, \Lambda}$ est une combinaison $\mathbb{C}$-linéaire de fonctions

$$
f^{l, k, I, \Lambda}=[\ln (u)]^{l}\left(u^{-\frac{1}{\alpha^{\Lambda}}\left(\gamma_{I}^{\Lambda}+\eta_{k}^{\Lambda}\right)}\right) \exp \left(\frac{-\beta_{I}^{\Lambda}}{\alpha^{\Lambda} u}\right),
$$

où

- $\alpha^{\Lambda}:=-(S+C) . \Lambda$, et $\beta_{I}^{\Lambda}:=A_{\Lambda} . I$, ainsi que $\gamma_{I}^{\Lambda}=B_{\Lambda} . I$;

- les $\eta_{k}^{\Lambda}$, pour $1 \leq k \leq k_{0}$, sont les valeurs propres de la matrice $\mathcal{M}^{\Lambda}$ (cf. (9.15));

- $l \leq l_{0}$, avec $l_{0}$ la taille maximale d'un bloc de Jordan d'une forme de Jordan de $\mathcal{M}^{\Lambda}$

(ii) La matrice $\mathcal{M}^{\Lambda}$ ne dépend que de $\Lambda$, pas de $I$, et ces coefficients s'écrivent chacun comme produit d'une coordonnée $R_{i}$ du multiindice $R$ et d'un nombre complexe complexe $b_{j}^{\Lambda}:=\sum_{i=1}^{n} \lambda_{i} b_{j i}$;

(iii) Pour $I=0$ on $a f_{i j}^{I, \Lambda}=0$.

Remarque. - Pour $I=0$ le système (9.4) n'est pas à singularité irrégulière, donc dans ce cas les seules solutions infiniment plates sont nulles. D'où la proposition 9.1 (iii).

Preuve de la proposition 8.2. - On suppose $\Lambda \in \mathfrak{B}$ (cf. lemme 8.1) fixé et $I \in \mathbb{D}$ (cf. définition 8.2). Pour $i \neq j$ on considérons les fonctions $f_{i j}^{\Lambda, I}$ telles que dans les propositions 8.2 et 9.1. Par la proposition 9.1 on sait qu'il existe une famille fini de nombres complexes $\omega_{i j}^{l, k, I, \Lambda}$ tels que pour $i \neq j$ on a

$$
f_{i j}^{\Lambda, I}(u)=\sum \omega_{i j}^{l, k, I, \Lambda}[\ln (u)]^{l}\left(u^{-\frac{1}{\alpha^{\Lambda}}\left(\gamma_{I}^{\Lambda}+\eta_{k}^{\Lambda}\right)}\right) \exp \left(\frac{-\beta_{I}^{\Lambda}}{\alpha^{\Lambda} u}\right) .
$$

DÉFinition 9.1. - On dit qu'une fonction $g: D S_{j}^{R}\left(r, r^{\prime}, \delta\right) \rightarrow \mathbb{C}, j=0,1$, est à paramètres $\kappa$ et $\xi$ si $g$ s'écrit $g=x^{I} f\left(x^{R}\right), I \in \mathbb{D}$, où il existe une famille finie de complexes $c_{0}, \ldots, c_{l_{0}}$ tels que

$$
f(u)=\left(\sum_{l \leq l_{0}} c_{l}[\ln (u)]^{l}\right)\left(u^{\kappa}\right) \exp \left(-\frac{\xi}{u}\right) .
$$

Les fonctions $f_{i j}^{\Lambda, I}$ s'écrivent de manière unique comme somme de fonctions à paramètres $\kappa$

$$
f_{i j}^{\Lambda, I}:=\sum_{\kappa \in \mathcal{K}^{I}} f_{i j}^{I, \Lambda, \kappa}
$$

TOME $137-2009-\mathrm{N}^{\mathrm{O}} 3$ 
où $\mathcal{K}^{I, \Lambda}=\left\{\gamma_{I}^{\Lambda}+\eta_{1}^{\Lambda}, \ldots, \gamma_{I}^{\Lambda}+\eta_{k_{0}}^{\Lambda}\right\}$. Les $f_{i j}^{I, \Lambda, \kappa}$ s'écrivent sous la forme

$$
\left(\sum_{l \leq l_{0}} c_{l}^{\Lambda}[\ln (u)]^{l}\right)\left(u^{\frac{1}{\alpha^{\Lambda}}\left(\gamma_{I}^{\Lambda}+\eta_{\kappa}^{\prime \Lambda}\right)}\right) \exp \left(-\frac{\beta_{I}^{\Lambda}}{\alpha^{\Lambda} u}\right) .
$$

Par l'identité de Jacobi on a la propriété $(\mathrm{P})$ suivante :

(P) : Soient $I \in \mathbb{D} \subset \mathbb{N}^{n}$ et $\xi_{0}, \kappa_{0} \in \mathbb{C}$ fixées. La somme des termes de $\left[\left(\Phi_{j}^{\Lambda}\right)^{*}\left(v_{\Lambda} \Pi\right),\left(\Phi_{j}^{\Lambda}\right)^{*}\left(v_{\Lambda} \Pi\right)\right], j=0,1$, s'écrivant sous la forme

$$
x^{I} \omega[\ln (u)]^{l} u^{\kappa_{0}} e^{-\frac{\xi_{0}}{u}} Y_{i} \wedge Y_{j} \wedge Y_{k},
$$

avec $u=x^{R}$, est nulle.

DÉfinition 9.2. - Un champ de trivecteurs sur $D S_{j}^{R}\left(r, r^{\prime}, \delta\right)$ est de paramètre $\kappa$ si il s'écrit comme somme de termes $f_{i j k} Y_{i} \wedge Y_{j} \wedge Y_{k}$, où $f_{i j k}$ est une fonction de paramètre $\kappa$.

On montre la proposition 8.2 en assurant, par l'appartenance de $\Lambda$ à un ensemble adéquat, que la propriété $(\mathrm{P})$ implique

$$
\begin{aligned}
0 & =\left[\Pi^{2}, x^{I_{0}} \Pi^{I_{0}, \Lambda}\right] \\
& =\left[\Pi^{2}, x^{I_{0}} \frac{1}{2} \sum_{i \neq j} f_{i j}^{I_{0}, \Lambda} Y_{i} \wedge Y_{j}\right],
\end{aligned}
$$

où $I_{0} \in \mathbb{D}$ tel que dans la proposition 8.2 (a priori cet $I_{0}$ dépend de $\Lambda$ ).

Termes en $x^{I_{0}}$ • - De l'identité de Jacobi $\left[\left(\Phi_{j}^{\Lambda}\right)^{*}\left(v_{\Lambda} \Pi\right),\left(\Phi_{j}^{\Lambda}\right)^{*}\left(v_{\Lambda} \Pi\right)\right], j=0,1$, il suit la relation

$$
\begin{aligned}
& {\left[\Pi^{2}, x^{I_{0}} \Pi^{I_{0}}\left(x^{R}\right)\right]+\sum_{\left(I^{\prime}, I^{\prime \prime}\right) \in \mathbb{E}}\left[x^{I^{\prime}} \Pi^{I^{\prime}, \Lambda}\left(x^{R}\right), x^{I^{\prime \prime}} \Pi^{I^{\prime \prime}, \Lambda}\left(x^{R}\right)\right]} \\
& =\left[\Pi^{2}, x^{I_{0}} \frac{1}{2} \sum_{i \neq j} f_{i j}^{\Lambda, I}\left(x^{R}\right) Y_{i} \wedge Y_{j}\right] \\
& \quad+\sum_{\left(I^{\prime}, I^{\prime \prime}\right) \in \mathbb{E}}\left[x^{I^{\prime}} \frac{1}{2} \sum_{i \neq j} f_{i j}^{\Lambda, I^{\prime}}\left(x^{R}\right) Y_{i} \wedge Y_{j}, x^{I^{\prime \prime}} \frac{1}{2} \sum_{i \neq j} f_{i j}^{\Lambda, I^{\prime \prime}}\left(x^{R}\right) Y_{i} \wedge Y_{j}\right]
\end{aligned}
$$

$(9.21)=0$,

où $\mathbb{E}:=\left\{\left(I^{\prime}, I^{\prime \prime \prime}\right) \in \mathbb{D} \times \mathbb{D} \mid \exists p \in \mathbb{N}^{*} x^{I^{\prime}+I^{\prime \prime}}=x^{I_{0}} x^{p R}\right\}$. Posons

$\mathrm{Cr}_{1}\left(i, j, \kappa_{1}, \Lambda\right):=\left[\Pi^{2}, x^{I} f_{i j}^{I_{0}, \Lambda, \kappa_{1}} Y_{i} \wedge Y_{j}\right], \quad \mathrm{Cr}_{2}\left(i, j, \kappa_{1}, \Lambda\right):=\left[x^{R} \Pi^{R}, x^{I} f_{i j}^{I_{0}, \Lambda, \kappa_{1}} Y_{i} \wedge Y_{j}\right]$. 
Soit $\left(I^{\prime}, I^{\prime \prime}\right) \in \mathbb{E}$. Posons

$$
\mathrm{Cr}_{3}\left(I^{\prime}, I^{\prime \prime}, k, m, t, q, \kappa_{2}, \kappa_{3}, \Lambda\right):=\left[x^{I^{\prime}} f_{k m}^{I^{\prime}, \Lambda, \kappa_{2}} Y_{k} \wedge Y_{m}, x^{I^{\prime \prime}} f_{t q}^{I^{\prime \prime}, \Lambda, \kappa_{3}} Y_{t} \wedge Y_{q}\right] .
$$

La proposition 8.2 suit du lemme 9.3 ci-dessous. En effet, pour $\Lambda \in \mathcal{B}-\mathcal{C}$, la propriété $(\mathrm{P})$ implique par bilinéarité du crochet de Schouten la relation

$$
\left[\Pi^{2}, x^{I_{0}} \frac{1}{2} \sum_{i \neq j} f_{i, j}^{I_{0}, \Lambda} Y_{i} \wedge Y_{j}\right]=0 .
$$

LEMME 9.3. - Il existe $\mathcal{C} \subset \mathcal{B}$ de mesure de Lebesque nulle tel que les champs de trivecteurs $\mathrm{Cr}_{2}\left(i, j, \kappa_{1}, \Lambda\right)$ et $\mathrm{Cr}_{3}\left(I^{\prime}, I^{\prime \prime}, k, m, t, q, \kappa_{2}, \kappa_{3}, \Lambda\right)$ ont un paramètre $\kappa$ différent de celui de $\operatorname{Cr}_{1}\left(i, j, \kappa_{1}, \Lambda\right)$, pour tout $\Lambda \in \mathcal{B}-\mathcal{C}$, pour tout $1 \leq i, j \leq n$ et $\kappa_{1} \in \mathcal{K}^{I_{0}, \Lambda}$, pour tout $\left(I^{\prime}, I^{\prime \prime}\right) \in \mathbb{E}$, tout $1 \leq k, m \leq n$ et $\kappa_{2} \in \mathcal{K}^{I^{\prime}, \Lambda}$, et tout $1 \leq t, q \leq n, \kappa_{3} \in \mathcal{K}^{I^{\prime \prime}, \Lambda}$.

On vérifie facilement le lemme suivant.

LEMme 9.4. - Soit $\kappa$ fixé et soit $f=\left[\ln \left(x^{R}\right)\right]^{l}\left(\left(x^{R}\right)^{\kappa}\right) \exp \left(-\frac{\xi}{x^{R}}\right)$ une fonction de paramètre $\kappa$. Soit $L$ un champ de vecteurs linéaire diagonal. Alors la dérivée de Lie $\mathcal{L}_{L}(f)$ est également fonction de paramètre $\kappa$.

Preuve du lemme 9.3. - D'après le lemme 9.4, relations (2.6), (2.8)-(2.10), ainsi que définitions 9.1 et 9.2 , le paramètre $\kappa$ de $\mathrm{Cr}_{1}\left(i, j, \kappa_{1}, \Lambda\right)$, c'est-à-dire le paramètre $\kappa$ de $\left[\Pi^{2}, x^{I_{0}} f_{i j}^{I_{0}, \Lambda, \kappa_{1}} Y_{i} \wedge Y_{j}\right]$ vaut $\kappa_{1}$.

Egalement d'après le lemme 9.4, les relations (2.6), (2.8)-(2.10), ainsi que les définitions 9.1 et 9.2 , le paramètre $\kappa$ de $\mathrm{Cr}_{2}\left(i, j, \kappa_{1}, \Lambda\right):=\left[x^{R} \Pi^{R}, x^{I_{0}} f_{i j}^{I_{0}, \Lambda, \kappa_{1}} Y_{i} \wedge\right.$ $Y_{j}$ ] vaut $1+\kappa_{1}$. (Il vaut $1+\kappa_{1}$ et non $\kappa_{1}$ puisque $\left.I \in \mathbb{D}\right)$.

Soit $\kappa_{\mathrm{Cr}_{3}}$ le paramètre $\kappa$ de $\mathrm{Cr}_{3}\left(I^{\prime}, I^{\prime \prime}, k, m, t, q, \kappa_{1}, \kappa_{2}\right)$. Comme $\left(I^{\prime}, I^{\prime \prime}\right) \in \mathbb{E}$, il existe $e \in \mathbb{N}^{*}$ tel qu'on a $I^{\prime}+I^{\prime \prime}=I_{0}+e R$. Puisque

$$
\mathrm{Cr}_{3}\left(I^{\prime}, I^{\prime \prime}, k, m, t, q, \kappa_{2}, \kappa_{3}, \Lambda\right):=\left[x^{I^{\prime}} f_{k m}^{I^{\prime}, \Lambda, \kappa_{1}} Y_{k} \wedge Y_{m}, x^{I^{\prime \prime}} f_{t q}^{I^{\prime \prime}, \Lambda, \kappa_{3}} Y_{t} \wedge Y_{q}\right] \text {, }
$$

on calcule comme dans le cas du deuxième crochet que

$$
\kappa_{\mathrm{Cr}_{3}}=\kappa_{2}+\kappa_{3}+e .
$$

Pour montrer le lemme 9.3 il reste donc à montrer que $\kappa_{\mathrm{Cr}_{3}}$ vérifie

$$
\kappa_{\mathrm{Cr}_{3}} \neq \kappa_{1} \text {. }
$$

D'après la proposition 9.1, il existe trois valeurs propres $\eta_{1}^{\Lambda}, \eta_{2}^{\Lambda}$ et $\eta_{3}^{\Lambda}$ de la matrice $\mathcal{M}^{\Lambda}$ telles que

$$
\kappa_{1}=\frac{1}{\alpha^{\Lambda}}\left(\gamma_{I_{0}}^{\Lambda}+\eta_{1}^{\Lambda}\right), \kappa_{2}=\frac{1}{\alpha^{\Lambda}}\left(\gamma_{I^{\prime}}^{\Lambda}+\eta_{2}^{\Lambda}\right), \kappa_{3}^{\Lambda}=\frac{1}{\alpha^{\Lambda}}\left(\gamma_{I^{\prime \prime}}^{\Lambda}+\eta_{3}^{\Lambda}\right) .
$$

Rappelons également que d'après la proposition 9.1 on a $\alpha^{\Lambda}:=-(S+C) . \Lambda$ et

$$
\gamma_{I}^{\Lambda}=B_{\Lambda} \cdot I
$$

TOME $137-2009-\mathrm{N}^{\circ} 3$ 
D'après (9.22) et (9.24), il suit $\kappa_{C r_{3}}=e+\frac{1}{\alpha^{\Lambda}}\left(B_{\Lambda} \cdot\left(I^{\prime}+I^{\prime \prime}\right)+\eta_{2}^{\Lambda}+\eta_{3}^{\Lambda}.\right)$ Le but est d'avoir la relation (9.23). En utilisant la relation précédente, (9.25) et (9.24), la relation (9.23) s'écrit

$$
e+\frac{1}{\alpha^{\Lambda}}\left(B_{\Lambda} \cdot\left(I^{\prime}+I^{\prime \prime}\right)+\eta_{2}^{\Lambda}+\eta_{3}^{\Lambda} \cdot\right) \neq \frac{1}{\alpha^{\Lambda}}\left(B_{\Lambda} \cdot I_{0}+\eta_{1}^{\Lambda}\right) .
$$

On en déduit que lemme 9.3 suit du lemme 9.5 ci-dessous.

Lemme 9.5. - Il existe un ensemble $\mathcal{C} \subset \mathcal{B}$ de mesure de Lebesque nulle, tel que

$$
e+\frac{1}{\alpha^{\Lambda}}\left(B_{\Lambda} \cdot\left(I^{\prime}+I^{\prime \prime}\right)+\eta_{i}^{\Lambda}+\eta_{j}^{\Lambda} \cdot\right) \neq \frac{1}{\alpha^{\Lambda}}\left(B_{\Lambda} \cdot I+\eta_{k}^{\Lambda}\right)
$$

pour tout $\Lambda \in \mathcal{B}-\mathcal{C}, 1 \leq i, j, k \leq n, I \in \mathbb{D}$ et toute paire de multiindices $I^{\prime}, I^{\prime \prime} \in \mathbb{D}$ de la forme $I^{\prime}+I^{\prime \prime}=e R+I, e \in \mathbb{N}^{*}$.

Preuve du lemme 9.5. - Rappelons d'abord $B_{\Lambda}=(S . \Lambda) C-(C . \Lambda) S$. Supposons qu'on a $I^{\prime}+I^{\prime \prime}=I+e R$, avec $e \in \mathbb{N}^{*}$. Alors on a

$$
\begin{aligned}
B_{\Lambda} \cdot\left(I^{\prime}+I^{\prime \prime}\right) & =B_{\Lambda} \cdot(I+e R) \\
& =B_{\Lambda} \cdot I+(S . \Lambda) e(R . C),
\end{aligned}
$$

car $S . R=0$. Rappelons que d'après la proposition 9.1, $\alpha^{\Lambda}=-(S+C) . \Lambda$. D'après (9.27) et (9.28) il suffit pour établir le lemme 9.5 qu'on assure par l'appartenance de $\Lambda$ à un sous-ensemble de $\mathfrak{B}$ de mesure pleine la relation

$$
\frac{e(S . \Lambda)(R . C)+\eta_{r}^{\Lambda}+\eta_{s}^{\Lambda}-\eta_{t}^{\Lambda}}{(S+C) . \Lambda} \neq e
$$

pour tout $e \in \mathbb{N}$ et tout triplet $\eta_{r}^{\Lambda}, \eta_{s}^{\Lambda}$ et $\eta_{t}^{\Lambda}$ de valeurs propres de $\mathcal{M}^{\Lambda}$.

La matrice $\mathcal{M}^{\Lambda}$ dépend polynomialement du paramètre $\Lambda=\left(\Lambda_{1}, \ldots, \Lambda_{n}\right)$ (cf. (9.15),(9.4)). Son polynôme caractéristique $P:(\Lambda, x) \rightarrow P(\Lambda, x)$ est alors polynomial en $x$ et en $\Lambda$. Recouvrons un sous-ensemble de mesure pleine de $\overline{\mathfrak{B}}$ par des ouverts $U$ permettant de choisir les déterminations des racines de $P$ telles que les valeurs propres de $\mathcal{M}^{*}$ constituent des fonctions analytiques $\Lambda \rightarrow \eta_{i}^{\Lambda}, i=1, \ldots, n(n-1)$ sur $U$. Pour prouver le lemme 9.5 il suffit de montrer qu'il existe $V \subset U$ de mesure de Lebesque nulle tel que relation (9.29) soit satisfaite pour $\Lambda \in U \backslash V$.

On considère premièrement l'ensemble $\mathcal{E}$ des triplets $\left(\eta_{r}^{\Lambda}, \eta_{s}^{\Lambda}, \eta_{t}^{\Lambda}\right)$ de valeurs propres de $\mathcal{M}^{\Lambda}$ telles qu'il n'existent pas $c_{r s t}^{1}, c_{r s t}^{2} \in \mathbb{C}, c_{r s t}^{2} \neq 0$ avec $\eta_{r}^{\Lambda}+\eta_{s}^{\Lambda}-$ $\eta_{t}^{\Lambda}=c_{r s t}^{1}(S . \Lambda)+c_{r s t}^{2}(C . \Lambda)$.

Deuxièmement on considère l'ensemble $\mathcal{E}^{c}$ des triplets de valeurs propres $\left(\eta_{r}^{\Lambda}, \eta_{s}^{\Lambda}, \eta_{t}^{\Lambda}\right)$ telles qu'il existe $c_{r s t}^{1}, c_{r s t}^{2} \in \mathbb{C}, c_{r s t}^{2} \neq 0$ avec $\eta_{r}^{\Lambda}+\eta_{s}^{\Lambda}-\eta_{t}^{\Lambda}=$ $c_{r s t}^{1}(S . \Lambda)+c_{r s t}^{2}(C . \Lambda)\left(\mathcal{E}\right.$ ou $\mathcal{E}^{c}$ peuvent être vide). 
Posons $V:=\cup_{e \in \mathbb{N}^{*}}\left(V_{1}^{e} \cup V_{2}^{e}\right)$ où pour $e \in \mathbb{N}^{*}, V_{1}^{e}$ est tel que sur $U \backslash V_{1}^{e}$ on a (9.29) pour les triplets de valeurs propres appartenant à $\mathcal{E}$, et $V_{2}^{e}$ est tel que sur $U \backslash V_{2}^{e}$ on a (9.29) pour les triplets de valeurs propres appartenant à $\mathcal{E}^{c}$.

Reste à montrer : pour tout $e \in \mathbb{N}$, les ensembles $V_{1}^{e}$ et $V_{2}^{e}$ sont de mesure de Lebesque nulle. Soit $e \in \mathbb{N}^{*}$. Considérons $g: W \rightarrow \mathbb{C}$ définie par

$$
g(\Lambda)=\frac{e(S . \Lambda)(R . C)+\eta_{r}^{\Lambda}+\eta_{s}^{\Lambda}-\eta_{t}^{\Lambda}}{(S+C) . \Lambda}+e,
$$

où $W:=\{\Lambda \in U \mid(S+C) . \Lambda \neq 0\}$. Pour $\left(\eta_{r}^{\Lambda}, \eta_{s}^{\Lambda}, \eta_{t}^{\Lambda}\right) \in \mathcal{E}, g$ est holomorphe et non-constante. L'ensemble des $V_{1}^{e}:=\{\Lambda \in W \mid g(\Lambda)=0\}$ est donc de mesure de Lebesque nulle.

On considère maintenant $\left(\eta_{r}^{\Lambda}, \eta_{s}^{\Lambda}, \eta_{t}^{\Lambda}\right) \in \mathcal{E}^{c}$. Il suit de la proposition 9.1 (ii) que la multiplication de $\Pi$ par un scalaire $\nu$ multiplie $\Pi^{R}=S \wedge C$ - et donc les valeurs propres $\eta_{r}^{\Lambda}, \eta_{s}^{\Lambda}, \eta_{t}^{\Lambda}$ par $\nu$. On en déduit que $c_{r s t}^{1}$ et $c_{r s t}^{2}$ ne peuvent pas être non-nuls en même temps. En effet, si $c_{r s t}^{1}$ et $c_{r s t}^{2}$ sont simultanément non nuls on a pour tout $\nu \in \mathbb{C}$

$$
\begin{aligned}
\nu\left(\eta_{r}^{\Lambda}+\eta_{s}^{\Lambda}-\eta_{t}^{\Lambda}\right) & =c_{r s t}^{1}(\nu S . \Lambda)+c_{r s t}^{2}(C . \Lambda) \\
& =c_{r s t}^{1}(S . \Lambda)+c_{r s t}^{2}(\nu C . \Lambda),
\end{aligned}
$$

ce qui est absurde. Comme $S$ et $C$ ne sont pas colinéaires, en en déduit que $g$ est holomorphe et non identiquement nulle. L'ensemble $V_{2}^{e}:=\{\Lambda \in W \mid g(\Lambda)=0\}$ est donc de mesure de Lebesque nulle.

\section{Classification analytique}

On reproduit de [16] (avec quelques modifications) des définitions et propriétés premières de certains faisceaux de transformations sectorielles. Soit $S^{1} \subset \mathbb{C}$ le cercle unité, et $U \subset S^{1}$ connexe. On désigne aussi par $U$ le secteur de $\mathbb{C}$, de sommet 0 , formé des points $z \neq 0$ tel que $\frac{z}{|z|} \in U$ et $|z|<R$ (le rayon n'est pas précisé en général). On dit que le secteur $U$ est ouvert (resp. fermé) si l'arc qui le représente dans $S^{1}$ est ouvert (resp. fermé).

Faisceaux. - Soit $\alpha \in S^{1}$. On considère l'ensemble $V_{\alpha}$ des germes en $0 \in \mathbb{C} \times \mathbb{C}^{n}$ de fonctions analytiques $f: V_{\alpha} \times \Delta \rightarrow \mathbb{C}, \mathcal{C}^{\infty}$ au sens de Whitney (cf. [10], [20]) sur $V \times \Delta$, où on désigne par $V_{\alpha}=V_{\alpha}(r, \theta)$ le secteur fermé bissecté par $\alpha \mathbb{R}^{+}$ de rayon $r$ et d'ouverture $2 \theta$, et par $\Delta$ un polydisque fermé de centre $0 \in \mathbb{C}^{n}$.

Les fonctions $f$ peuvent être considérés comme des fonctions analytiques admettant un développement asymptotique fort (cf. section 2) : en effet, le jet

TOME $137-2009-\mathrm{N}^{\circ} 3$ 
infini de $f$ le long de $\{0\} \times \Delta$ s'interprète comme une série formelle en $z \in V$ avec coefficient à paramètre $x \in \Delta$

$$
\hat{f}(z, x)=\sum_{n \geq 0} f_{n}(x) z^{n},
$$

où les coefficients $f_{n}$ sont analytiques sur un même polydisque de centre $0 \in \mathbb{C}^{n}$. Si on note $\hat{f}^{k}=\sum_{n \geq 0}^{k} f_{n}(x) z^{n}$, on a pour tout $k, l \in \mathbb{N}, \bar{V} \subset V$ secteur fermé et $K \subset \Delta$ compact

$$
\left|z^{-k+1}\right|\left|\frac{\partial^{l} f}{\partial^{r} z}(z, x)-\frac{\partial^{l} \hat{f}^{k}}{\partial^{l} z}(z, x)\right| \stackrel{z \rightarrow 0}{\longrightarrow} 0
$$

uniformément sur $\bar{V} \times K$.

On définit $\widehat{\mathcal{B}}$ comme l'anneau des séries formelles de forme (10.1), et $\mathcal{B}$ le faisceaux d'anneaux sur $S^{1}$ tel pour tout $\alpha \in S^{1}$, la fibre en $\alpha$ soit l'ensemble $V_{\alpha}$. On note $\mathcal{B}_{\alpha}$ la fibre en $\alpha \in S^{1}$ de $\mathcal{B}$.

Soit $\mathcal{D}_{R, \Lambda}$ le faisceau de groupes (dit des transformations sectorielles) sur $S^{1}$ dont la fibre en $\alpha \in S^{1}$ est constituée des transformations $\Phi_{\alpha}:\left(z, x_{1}, \ldots, x_{n}\right) \rightarrow$ $\left(z, x_{1} e^{f_{1}}, \ldots, e^{f_{n}}\right)$, avec $f_{i} \in \mathcal{B}_{\alpha}, f_{i}(0, x)=0$ et tel que $x^{R} \circ \Phi_{\alpha}=x^{R}, x^{\Lambda} \circ \Phi_{\alpha}=$ $x^{\Lambda}$.

On note $\mathcal{D}_{E p, \Lambda}^{\infty} \subset \mathcal{D}_{R, \Lambda}$ le sous-faisceau (dit des transformations infiniment plates) tel que les transformations $\Phi_{\alpha}$ soient infiniment plates en $z$ le long de $\{0\} \times \Delta$ (les $f_{i}$ sont alors également infiniment plats) .

Cochaines, cocycles et isomorphisme. - Soit $\mathcal{U}=\left\{U_{1}, \ldots, U_{m}\right\}$ un recouvrement ouvert de $S^{1}$ tel que les $U_{i}$ soient des arcs de longueur inférieure ou égale à $2 \pi, U_{i, i+1} \equiv U_{i} \cap U_{i+1} \neq \varnothing$.

On note $C^{0}\left(\mathcal{U}, \mathcal{D}_{R, \Lambda}\right)$ ( 0 -cochaînes) le groupe des applications qui à $U_{i} \in \mathcal{U}$ font correspondre une section $\Phi_{i}$ du faisceau $\mathcal{D}_{R, \Lambda}$ et telles que pour tout $1 \leq$ $i, j \leq m$ on a $\hat{\Phi}_{i}=\hat{\Phi}_{j}$.

On note $C^{1}\left(\mathcal{U}, \mathcal{D}_{R, \Lambda}^{\infty}\right)$ (1-cochaînes) le groupe des applications qui à tout $U_{i} \cap$ $U_{j} \neq \varnothing$ font correspondre une section $\Psi_{i j}$ du faisceau $\mathcal{D}_{R, \Lambda}^{\infty}$ des transformations infiniment plates. Soit $Z^{1}\left(\mathcal{U}, \mathcal{D}_{R, \Lambda}^{\infty}\right) \subset C^{1}\left(\mathcal{U}, \mathcal{D}_{R, \Lambda}^{\infty}\right)$ (1-cochaînes) tel qu'on a $\Psi_{i j} \circ \Psi_{j k}=\Psi_{i k}$ sur $U_{i} \cap U_{j} \cap U_{k} \neq \varnothing$ (Pour les raisons de ces dénominations cf. [16] et à la bibliographie qui s'y trouve).

On définit l'application cobord de Cěch $\partial: C^{0}\left(\mathcal{U}, \mathcal{D}_{R, \Lambda}\right) \rightarrow Z^{1}\left(\mathcal{U}, \mathcal{D}_{R, \Lambda}^{\infty}\right)$ par $\partial\left\{\Phi_{i}\right\}=\left\{\Phi_{i} \Phi_{j}^{-1}\right\}$ Pour la preuve du résultat suivant du à B. Malgrange et repris par Martinet-Ramis, on renvoie à [11].

ThÉORÈme 10.1. - L'application d est surjective. 
On considère maintenant la restriction à la surface $z=x^{R}$ de ce qui précède. Soit $\hat{B}$ l'anneau des séries formelles de la forme

$$
\hat{f}=\sum_{n \geq 0} f_{n}(x)\left(x^{R}\right)^{n}
$$

où les coefficients $f_{n}$ sont analytiques sur un même polydisque de $\mathbb{C}^{n}$ centré en 0 . Posons $\pi: \mathbb{C}^{n} \rightarrow \mathbb{C}$ défini par $\pi(x)=x^{R}$. Soit $B$ le faisceau d'anneaux sur $S^{1}$ défini ainsi : pour tout $\alpha \in S^{1}$, un élément de la fibre $B_{\alpha}$ en $\alpha$ est le germe en $0 \in \mathbb{C}^{n}$ d'une fonction $f: \pi^{-1}(U) \cap \Delta \rightarrow \mathbb{C}$, où $U \equiv U_{\alpha}(r, \theta) \subset \mathbb{C}$ est un secteur fermé bissecté par $\alpha \mathbb{R}^{+}$, d'ouverture $2 \theta$ et de rayon $r ; \Delta \in \mathbb{C}^{n}$ est un polydisque fermé de centre 0 . On suppose la fonction $f$ analytique sur l'intérieur de $\pi^{-1}(U) \cap \Delta$. Let jet infini d'une telle fonction le long de $E \cap \Delta$ est un élément de $\hat{B}$, c'est-à-dire que $f$ admet un développement asymptotique fort (cf. section 2) en le monôme $x^{R}$. On note $B^{\infty}$ le faisceau des fonctions infiniment plates sur $\pi^{-1}(U) \cap \Delta$. Soit le faisceau $D_{R, \Lambda}$ de groupes (non-abélien) sur $S^{1}$ dont la fibre en $\alpha \in S^{1}$ est constituée des transformations

$$
\left(x_{1}, \ldots, x_{n}\right) \mapsto\left(x_{1} e^{f_{1}}, \ldots, x_{n} e^{f_{n}}\right)
$$

avec pour tout $1 \leq i \leq n, f_{i} \in B_{\alpha}$ et $\sum_{i=1}^{n} R_{i} f_{i}=0, \sum_{i=1}^{n} \Lambda_{i} f_{i}=0$. On désigne par $D_{R, \Lambda}^{\infty} \subset D_{R, \Lambda}$ le sous-faisceau (dit des transformations infiniment plates) défini par la condition supplémentaire que pour tout $1 \leq i \leq n, f_{i}$ est infiniment plat sur $\pi^{-1}(U) \cap \Delta$. On désigne par $\hat{D}_{R, \Lambda}$ le groupe des transformations formelles de $\mathbb{C} \times \mathbb{C}^{n}$ en 0 de la forme

$$
\left(x_{1}, \ldots, x_{n}\right) \mapsto\left(x_{1} e^{f_{1}}, \ldots, x_{n} e^{f_{n}}\right)
$$

avec pour tout $1 \leq i \leq n, f_{i} \in \hat{B}$ et $\sum_{i=1}^{n} R_{i} f_{i}=0, \sum_{i=1}^{n} \Lambda_{i} f_{i}=0$.

Soit $C^{0}\left(\mathcal{U}, D_{R, \Lambda}\right)$ ( 0 -cochaînes) le groupe des applications qui à $U_{i} \in \mathcal{U}$ font correspondre une section $\Phi_{i}$ du faisceau $D_{R, \Lambda}$ et telles que pour tout $1 \leq i, j \leq m$ on a $\hat{\Phi}_{i}=\hat{\Phi}_{j}$.

On définit alors comme plus haut $C^{0}\left(\mathcal{U}, D_{R, \Lambda}\right), C^{1}\left(\mathcal{U}, D_{R, \Lambda}^{\infty}\right)$ ainsi que $Z^{1}\left(\mathcal{U}, D_{R, \Lambda}^{\infty}\right)$. La preuve du résultat qui suit est identique à celle de [12] (corollaire 4.6, p. 603).

Corollaire 10.1. - L'applicartion $\partial: \mathcal{C}^{0}\left(\mathcal{U}, \mathcal{D}_{R, \Lambda}^{\infty}\right) \rightarrow Z^{1}\left(\mathcal{U}, D_{R, \Lambda}^{\infty}\right)$ est surjective.

Soient $\left\{f_{i}\right\}_{i=1, \ldots, n}$ des fonctions analytiques bornées sur $\pi^{-1}\left(U_{i}\right) \cap \Delta$ admettant toutes $\hat{f}$ comme développement asymptotique au sens de Gérard-Sibuya dans $\pi^{-1}\left(U_{i}\right) \cap \Delta$. Posons $f_{i, i+1}=f_{i} \circ f_{i+1}^{-1}$ dans $\pi^{-1}\left(U_{i, i+1}\right) \cap \Delta$. Les $f_{i, i+1}$ admettent la fonction nulle comme développement asymptotique au sens de Gérard-Sibuya. La preuve du résultat qui suit est identiques à [11] (propositions I.6.2, I.6.3, p. 91-92). 
Proposition 10.1. - Supposons que $\left.\left\{f_{i, i+1}\right)\right\} \in Z^{1}\left(\mathcal{U}, B^{\infty}\right)$. Alors $\hat{f}$ appartient $\grave{a} \hat{B}$.

Dans le reste de cette section on considère une forme normale polynomiale fixée $\Pi_{n}=\Pi^{2}+x^{R} \Pi^{R}$ telle que dans le théorème 3.1 (de normalisation sectorielle). Le but est de donner une classification analytique des structures de Poisson holomorphes formellement conjuguées à $\Pi_{n}$, à multiplication par une unité analytique près. Soit $0<\epsilon<\pi / 2$ fixé quelconque. D'après le théorème 3.1 on a $\Lambda, \delta$ tels que pour toute structure de Poisson holomorphe de forme normale formelle $\Pi_{n}$ il existe $v_{\Lambda}, r, r^{\prime}>0$ et $\Phi_{j}^{\Lambda}, j=0,1$ tels que sur $D S_{j}^{R}$ on a $\left(\Phi_{j}^{\Lambda}\right)^{*}\left(v_{\Lambda} \Pi\right)=\Pi_{n}$. Fixons maintenant le recouvrement ouvert $\mathcal{U}$ de $S^{1}$

$$
\mathcal{U}=\left\{U_{j}\right\}_{j=0,1} \quad U_{j}=\left\{\theta \in S^{1} ;|\theta-[\arg \delta+\pi(j+1 / 2)]|<\pi-\epsilon\right\} .
$$

Définition 10.1. - On dit que $\Phi_{01} \in D_{R, \Lambda}^{\infty}$ est une isotropie sectorielle de $\Pi_{n}$ si on a $\Phi_{01}^{*}\left(\Pi_{n}\right)=\Pi_{n}$. On dit que $\Phi_{01} \in D_{R, \Lambda}^{\infty}$ est une isotropie sectorielle de $\frac{1}{x^{\Lambda}} X_{\Pi_{n}}\left(x^{\Lambda}\right)$ si $\Phi_{01}^{*}\left(\frac{1}{x^{\Lambda}} X_{\Pi_{n}}\left(x^{\Lambda}\right)\right)=\frac{1}{x^{\Lambda}} X_{\Pi_{n}}\left(x^{\Lambda}\right)$.

Désignons par $D_{R, \Lambda, \Pi_{n}} \subset D_{R, \Lambda}^{\infty}$ le sous-faisceau des transformations isotropes de $\Pi_{n}$, et par $D_{R, \Lambda,\left[\frac{1}{x \Lambda} X_{\Pi_{n}}\left(x^{\Lambda}\right)\right]} \subset D_{R, \Lambda}^{\infty}$ le sous-faisceau des transformations isotropes de $\frac{1}{x^{\Lambda}} X_{\Pi_{n}}\left(x^{\Lambda}\right)$.

Proposition 10.1. - On a $D_{R, \Lambda, \Pi_{n}}=D_{R, \Lambda,\left[\frac{1}{x^{\Lambda}} X_{\Pi_{n}}\left(x^{\Lambda}\right)\right]}$.

Preuve de la proposition 10.1. - Soit $\Phi \in D_{R, \Lambda}^{\infty}$. Comme $x^{\Lambda} \circ \Phi=x^{\Lambda}$, on a

$$
\Phi^{*}\left(\frac{1}{x^{\Lambda}} X_{\Pi_{n}}\left(x^{\Lambda}\right)\right)=\frac{1}{x^{\Lambda}} X_{\Phi^{*}\left(\Pi_{n}\right)}\left(x^{\Lambda}\right) .
$$

Il suit $D_{R, \Lambda,\left[\frac{1}{x^{\Lambda}} X_{\Pi_{n}}\left(x^{\Lambda}\right)\right]} \subset D_{R, \Lambda, \Pi \text {. Montrons l'inclusion inverse. Supposons }}$ $\Phi \in D_{R, \Lambda,\left[\frac{1}{x^{\Lambda}} X_{\Pi_{n}}\left(x^{\Lambda}\right)\right]}$. On a $\Phi^{*}\left(\Pi_{n}\right)=\Pi_{n}+\mathcal{R}$ où $\mathcal{R}$ est tel que dans la proposition 8.1. En même temps $\frac{1}{x^{\Lambda}} X_{\Phi^{*}\left(\Pi_{n}\right)}\left(x^{\Lambda}\right)=\frac{1}{x^{\Lambda}} X_{\left(\Pi_{n}\right)}\left(x^{\Lambda}\right)$. On montre alors comme dans la preuve du théorème 3.1 que $\mathcal{R}=0$.

Définissons encore l'espace $H^{1}\left(\mathcal{U}, D_{R, \Lambda, \Pi_{n}}^{\infty}\right)$ dont on a besoin plus bas. Deux 1-cochaînes $\left\{\Psi_{i j}\right\}$ et $\left\{\Omega_{i j}\right\} \in C^{1}\left(\mathcal{U}, D_{R, \Lambda, \Pi_{n}}^{\infty}\right)$ sont dites cohomologues s'il existe une 0-cochaîne $\left\{\Phi_{i}\right\} \in C^{0}\left(\mathcal{U}, D_{R, \Lambda, \Pi_{n}}^{\infty}\right)$ tel que $\Omega_{i j}=\Phi_{i}^{-1} \Psi_{i j} \Phi_{i}$. Cela définit une relation d'équivalence sur $C^{1}\left(\mathcal{U}, D_{R, \Lambda, \Pi_{n}}^{\infty}\right)$ et on montre qu'elle respecte $Z^{1}\left(\mathcal{U}, D_{R, \Lambda, \Pi_{n}}^{\infty}\right)$ : si $\left\{\Psi_{i j}\right\}$ et $\left\{\Omega_{i j}\right\}$ sont cohomologues et si $\left\{\Psi_{i j}\right\}$ est un 1cocycle, alors $\left\{\Omega_{i j}\right\}$ est également un 1-cocycle. On définit $H^{1}\left(\mathcal{U}, D_{R, \Lambda, \Pi_{n}}^{\infty}\right)$ comme l'ensemble des classes d'équivalences dans $Z^{1}\left(\mathcal{U}, D_{R, \Lambda, \Pi_{n}}^{\infty}\right)$.

Puisque $\mathcal{U}$ est uniquement composé de deux ouverts on a $C^{1}\left(\mathcal{U}, D_{R, \Lambda, \Pi_{n}}^{\infty}\right)=$ $Z^{1}\left(\mathcal{U}, D_{R, \Lambda, \Pi_{n}}^{\infty}\right)$, et on montre comme dans [16, section 3.4], en utilisant la 
proposition 10.1, que $C^{0}\left(\mathcal{U}, D_{R, \Lambda, \Pi_{n}}^{\infty}\right)$ est réduit à la cochaîne triviale : l'identité de $\mathbb{C}^{n}$.

Rappelons $\frac{1}{x^{\Lambda}} X_{\Pi^{2}}\left(x^{\Lambda}\right):=\mu_{1} Y_{1}+\cdots+\mu_{n} Y_{n}$ et posons $N=\left(\mu_{1}, \ldots, \mu_{n}\right) \in$ $\mathbb{C}^{n}$. En utilisant la proposition 10.1 on obtient comme dans [16, prop. 3.4.3] :

Proposition 10.2. - On a

$$
H^{1}\left(\mathcal{U}, D_{R, \Lambda, \Pi_{n}}^{\infty}\right) \cong C^{1}\left(\mathcal{U}, D_{R, \Lambda, \Pi_{n}}^{\infty}\right) .
$$

De plus, cet espace s'identifie à l'espace des $2 n$ séries holomorphes au voisinage de $0 \in \mathbb{C}^{n}$ de la forme

$$
\left(\sum_{(-1)^{j}(Q, N) / \delta<0} a_{i, Q}^{j} x^{Q}\right)_{i=1, \ldots, n} j=0,1
$$

et $\sum_{i=1}^{n} R_{i} a_{i, Q}^{j}=0, \sum_{i=1}^{n} \Lambda_{i} a_{i, Q}^{j}=0$.

Désignons par $\mathfrak{E}_{\Pi_{\mathrm{n}}}$ l'ensemble des structures de Poisson holomorphes de forme normale formelle $\Pi_{n}$ telles que l'unité analytique $v_{\Lambda}$ fourni par le théorème 3.1 soit égale à la fonction constante 1 .

REMARque. - Par multiplication par une unité analytique adéquate, toute structure de Poisson telle que dans le théorème 3.1, peut être ramenée à une structure de Poisson appartenant à $\mathfrak{E}_{\Pi_{n}}$.

THÉORÈme 10.2. - L'espace des orbites de $D_{R, \Lambda}$ dans $\mathfrak{E}_{\Pi_{\mathrm{n}}}$ est en bijection avec $H^{1}\left(\mathcal{U}, D_{R, \Lambda, \Pi_{n}}^{\infty}\right)$.

Démonstration. - Soit $\diamond: \mathfrak{E}_{\Pi_{n}} \rightarrow C^{1}\left(\mathcal{U}, D_{R, \Lambda}^{\infty}, \Pi_{n}\right)=H^{1}\left(\mathcal{U}, D_{R, \Lambda}^{\infty}, \Pi_{n}\right)$ l'application qui associe à une structure de Poisson $\Pi \in \mathfrak{E}_{\Pi_{n}}$ l'isotropie sectorielle $\left\{\Phi_{0}^{\Lambda} \circ\left(\Phi_{1}^{\Lambda}\right)^{-1}\right\}$, où $\Phi_{j}^{\Lambda}, j=0,1$, est la transformation analytique sur $D S_{j}^{R}\left(r, r^{\prime}, \delta\right)$ fourni par le théorème 3.1 , conjuguant $\Pi$ à la forme normale $\Pi_{n}$.

Montrons que l'application $\diamond$ passe au quotient en une application injective $\bar{\diamond}: \mathfrak{E}_{\Pi_{n}} / D_{R, \Lambda} \rightarrow H^{1}\left(\mathcal{U}, D_{R, \Lambda}^{\infty}, \Pi_{n}\right)$. Soient $\Pi_{1}, \Pi_{2} \in \mathfrak{E}_{\Pi_{n}}$ analytiquement conjuguées par $\Psi \in D_{R, \Lambda}$ sur un voisinage $V$ de $0 \in \mathbb{C}^{n}$. Par le théorème 3.1, ils existent des transformations $\Phi_{j}^{\Lambda, 1}, \Phi_{j}^{\Lambda, 2} \in D_{R, \Lambda}, j=0,1$, uniques telles que $\left(\Phi_{j}^{\Lambda, 1}\right)^{*}\left(\Pi_{1}\right)=\Pi_{n}$ et $\left(\Phi_{j}^{\Lambda, 2}\right)^{*}\left(\Pi_{2}\right)=\Pi_{n}$ sur $D S_{j}^{R}\left(r, r^{\prime}, \delta\right) j=0,1$. On en déduit que sur $D S_{j}^{R}\left(r, r^{\prime}, \delta\right) \cap V, j=0,1$, on a $\Psi_{j}=\left(\Phi_{j}^{\Lambda, 2}\right)^{-1} \circ \Phi_{j}^{\Lambda, 1}$. On en déduit que l'application $\diamond$ passe bien au quotient.

Montrons l'injectivité. Supposons qu'on a $\diamond\left(\Pi_{1}\right)=\diamond\left(\Pi_{2}\right)$, avec $\Pi_{1}, \Pi_{2} \in$ $\mathfrak{E}_{\Pi_{n}}$, c'est-à-dire $\Phi_{0}^{\Lambda, 1} \circ_{1} \Phi^{\Lambda, 1}=\Phi_{0}^{\Lambda, 2} \circ \Phi_{1}^{\Lambda, 2}$ sur $\pi^{-1}\left(U_{0} \cap U_{1}\right) \cap \Delta:=$ $D S_{0}^{R}\left(r, r^{\prime}\right), \delta \cap D S_{1}^{R}\left(r, r^{\prime}, \delta\right)$. La transformation $\Psi_{j}:=\left(\Phi_{j}^{\Lambda, 2}\right)^{-1} \circ \Phi_{j}^{\Lambda, 1}$ est définie, analytique est bornée sur $\pi^{-1}\left(U_{j}\right) \cap \Delta$. Dans l'intersection $\pi^{-1}\left(U_{0} \cap U_{1}\right) \cap \Delta$ 
on a $\Psi_{1}=\Psi_{2}$. Les transformations $\Psi_{0}, \Psi_{0}$ se prolongent donc en une transformation $\Psi$ analytique et bornée sur $W \backslash\{0\}$, où $W$ désigne un voisinage de $0 \in \mathbb{C}^{n}$, et $\Psi$ se prolonge analytiquement en 0 . D'où $\Psi^{*}\left(\Pi_{2}\right)=\Pi_{1}$ sur $W$.

Reste à montrer la surjectivité de l'application $\bar{\diamond}$, i.e. à synthétiser une structure de Poisson $\Pi$ appartenant à $\mathfrak{E}_{\Pi_{n}}$ à partir d'une 1-cochaîne $\left\{\Psi_{01}\right\}$ sur $\mathcal{U}$ à valeurs dans $D_{R, \Lambda, \Pi_{n}}^{\infty}$ qui est aussi un cocycle - on rappelle $C^{1}\left(\mathcal{U}, D_{R, \Lambda, \Pi_{n}}^{\infty}\right)=$ $Z^{1}\left(\mathcal{U}, D_{R, \Lambda, \Pi_{n}}^{\infty}\right)$. D'après le corollaire 10.1, il existe une 0-chaîne $\left\{\Phi_{0}, \Phi_{1}\right\} \in$ $C^{0}\left(\mathcal{U}, D_{R, \Lambda}\right)$ telle que $\Phi_{1} \circ \Phi_{0}^{-1}=\Psi_{01}$ (rappelons que $\Phi_{1}$ et $\Phi_{2}$ admettent le même développement asymptotique fort en $\left.x^{R}\right)$.

Posons $\Pi_{i}=\left(\Phi_{i}^{-1}\right)^{*}\left(\Pi_{n}\right), i=0,1$. Sur $\pi^{-1}\left(U_{0} \cap U_{1}\right) \cap \Delta$ on a $\Pi_{0}=\Pi_{1}$, puisque $\left(\Phi_{0} \circ \Phi_{1}^{-1}\right)^{*}\left(\Pi_{n}\right)=\Pi_{n}$. Par conséquent $\Pi_{0}$ et $\Pi_{1}$ se prolongent en une structure de Poisson $\Pi$ analytique sur un voisinage de $0 \in \mathbb{C}^{n}$ et on vérifie que $\Pi$ satisfait les hypothèses du théorème 3.1 et que l'unité analytique associé est égale à 1 . D'où $\Pi \in \mathfrak{E}_{\Pi_{n}}$.

\section{BIBLIOGRAPHIE}

[1] V. I. ARNoL'D - Mathematical methods of classical mechanics, second éd., Graduate Texts in Math., vol. 60, Springer, 1989.

[2] B. Branksma \& L. Stolovitch - «Small divisors and large multipliers », Ann. Inst. Fourier (Grenoble) 57 (2007), p. 603-628.

[3] M. Chaperon - « Géométrie différentielle et singularités de systèmes dynamiques », Astérisque 138-139 (1986).

[4] J. F. Conn - « Normal forms for analytic Poisson structures », Ann. of Math. 119 (1984), p. 577-601.

[5] J.-P. Dufour \& A. WADE - « Formes normales de structures de Poisson ayant un 1-jet nul en un point », J. Geom. Phys. 26 (1998), p. 79-96.

[6] J.-P. Dufour \& N. T. Zung - Poisson structures and their normal forms, Progress in Mathematics, vol. 242, Birkhäuser, 2005.

[7] J. ÉCALLE - «Singularités non abordables par la géométrie », Ann. Inst. Fourier (Grenoble) 42 (1992), p. 73-164.

[8] R. GÉrard \& Y. SibuYa - «Étude de certains systèmes de Pfaff avec singularités », in Équations différentielles et systèmes de Pfaff dans le champ complexe (Sem., Inst. Rech. Math. Avancée, Strasbourg, 1975), Lecture Notes in Math., vol. 712, Springer, 1979, p. 131-288.

[9] P. Lohrmann - «Normalisation holomorphe de structures de Poisson », article soumis. 
[10] B. Malgrange - Ideals of differentiable functions, Tata Institute of Fundamental Research Studies in Mathematics, No. 3, Tata Institute of Fundamental Research, 1967.

[11] J. Martinet \& J.-P. Ramis - «Problèmes de modules pour des équations différentielles non linéaires du premier ordre », Publ. Math. I.H.É.S. 55 (1982), p. 63-164.

[12] _ _ « Classification analytique des équations différentielles non linéaires résonnantes du premier ordre », Ann. Sci. École Norm. Sup. 16 (1983), p. 571-621.

[13] A. Newlander \& L. NirenberG - « Complex analytic coordinates in almost complex manifolds », Ann. of Math. 65 (1957), p. 391-404.

[14] J.-P. RAMIS - «Les séries $k$-sommables et leurs applications », in Complex analysis, microlocal calculus and relativistic quantum theory (Proc. Internat. Colloq., Centre Phys., Les Houches, 1979), Lecture Notes in Phys., vol. 126, Springer, 1980, p. 178-199.

[15] L. Stolovitch - «Sur un théorème de Dulac », Ann. Inst. Fourier (Grenoble) 44 (1994), p. 1397-1433.

[16] , « Classification analytique de champs de vecteurs 1-résonnants de $\left(\mathbf{C}^{n}, 0\right) »$, Asymptotic Anal. 12 (1996), p. 91-143.

[17] _ «ingular complete integrability », Publ. Math. I.H.É.S. 91 (2000), p. 133-210.

[18] _ _ « Sur les structures de Poisson singulières », Ergodic Theory Dynam. Systems 24 (2004), p. 1833-1863.

[19] _ _ «ormalisation holomorphe d'algèbres de type Cartan de champs de vecteurs holomorphes singuliers », Ann. of Math. 161 (2005), p. 589612 .

[20] J.-C. Tougeron - Idéaux de fonctions différentiables, Springer, 1972, Ergebnisse der Mathematik und ihrer Grenzgebiete, Band 71.

[21] S. M. Voronin - «Analytic classification of germs of conformal mappings $(\mathbf{C}, 0) \rightarrow(\mathbf{C}, 0) »$, Funktsional. Anal. i Prilozhen. 15 (1981), p. 1-17, 96.

[22] A. Weinstein - «The local structure of Poisson manifolds », J. Differential Geom. 18 (1983), p. 523-557. 